\title{
Lorenz Ratios of Technically Important Metals and Alloys
}

EPARTMENT

U.S.

OF

COMMERCE

National

Bureau

QC

100

.45753

no. 634

1973

C.2 2 
The National Bureau of Standards ${ }^{1}$ was established by an act of Congress March 3, 1901. The Bureau's overall goal is to strengthen and advance the Nation's science and technology and facilitate their effective application for public benefit. To this end, the Bureau conducts research and provides: (1) a basis for the Nation's physical measurement system, (2) scientific and technological services for industry and government, (3) a technical basis for equity in trade, and (4) technical services to promote public safety. The Bureau consists of the Institute for Basic Standards, the Institute for Materials Research, the Institute for Applied Technology, the Center for Computer Sciences and Technology, and the Office for Information Programs.

THE INSTITUTE FOR BASIC STANDARDS provides the central basis within the United States of a complete and consistent system of physical measurement; coordinates that system with measurement systems of other nations; and furnishes essential services leading to accurate and uniform physical measurements throughout the Nation's scientific community, industry, and commerce. The Institute consists of a Center for Radiation Research, an Office of Measurement Services and the following divisions:

Applied Mathematics - Electricity - Mechanics - Heat - Optical Physics Linac Radiation ${ }^{2}$ - Nuclear Radiation " - Applied Radiation ${ }^{2}$ - Quantum Electronics: ${ }^{3}$ Electromagnetics $"$ - Time and Frequency ${ }^{3}$ - Laboratory Astrophysics : - Cryogenics ${ }^{3}$.

THE INSTITUTE FOR MATERIALS RESEARCH conducts materials research leading to improved methods of measurement, standards, and data on the properties of well-characterized materials needed by industry, commerce, educational institutions, and Government; provides advisory and research services to other Government agencies; and develops, produces, and distributes standard reference materials. The Institute consists of the Office of Standard Reference Materials and the following divisions:

Analytical Chemistry-Polymers-Metallurgy-Inorganic Materials-Reactor Radiation-Physical Chemistry.

THE INSTITUTE FOR APPLIED TECHNOLOGY provides technical services to promote the use of available technology and to facilitate technological innovation in industry and Government; cooperates with public and private organizations leading to the development of technological standards (including mandatory safety standards), codes and methods of test; and provides technical advice and services to Government agencies upon request. The Institute also monitors NBS engineering standards activities and provides liaison between NBS and national and international engineering standards bodies. The Institute consists of a Center for Building Technology and the following divisions and offices:

Engineering and Product Standards-Weights and Measures-Invention and Innovation-Product Evaluation Technology-Electronic Technology-Technical Analysis-Measurement Engineering-Building Standards and Code Services $^{4}$-Housing Technology ${ }^{4}$-Federal Building Technology ${ }^{4}$-Structures, Materials and Life Safety ${ }^{4}$-Building Environment ${ }^{4}$-Technical Evaluation and Application ${ }^{4}$ - Fire Technology.

THE INSTITUTE FOR COMPUTER SCIENCES AND TECHNOLOGY conducts research and provides technical services designed to aid Government agencies in improving cost effectiveness in the conduct of their programs through the selection, acquisition, and effective utilization of automatic data processing equipment; and serves as the principal focus within the executive branch for the development of Federal standards for automatic data processing equipment, techniques, and computer languages. The Center consists of the following offices and divisions:

Information Processing Standards-Computer Information-Computer Services -Systems Development-Information Processing Technology.

THE OFFICE FOR INFORMATION PROGRAMS promotes optimum dissemination and accessibility of scientific information generated within NBS and other agencies of the Federal Government; promotes the development of the National Standard Reference Data System and a system of information analysis centers dealing with the broader aspects of the National Measurement System; provides appropriate services to ensure that the NBS staff has optimum accessibility to the scientific information of the world, and directs the public information activities of the Bureau. The Office consists of the following organizational units:

Office of Standard Reference Data-Office of Technical Information and Publications-Library-Office of International Relations.

\footnotetext{
${ }_{1}$ Headquarters and Laboratories at Gaithersburg, Maryland, unless otherwise noted; mailing address Washington, D.C. 20234

Part of the Center for Radiation Research.

3 Located at Boulder, Colorado 80302 .

- Part of the Center for Building Technology.
} 
J.G. Hust

L.L. Sparks

Cryogenics Division

Instilute for Basic Standards

U.S. National Bureau of Standards

Boulder, Colorado 80302

t. Technical note no. 634

NBS Technical notes are designed to supplement the Bureau's regular publications program. They provide a means for making available scientific data that are of transient or limited interest. Technical Notes may be listed or referred to in the open literature.

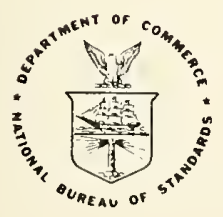

U.S. DEPARTMENT OF COMMERCE, Peter G. Peterson, Secretary NATIONAL BUREAU OF STANDARDS, Lawrence M. Kushner, Acting Director

Issued February 1973 


\section{National Bureau of Standards Technical Note 634}

Nat. Bur. Stand.(U.S.) Tech. Note 634,133 pages (February 1973) CODEN: NBTNAE

For sale by the Superintendent of Documents, U.S. Government Printing Office, Washington, D. C. 20402 (Order by SD Catalog No. C13.46:634). 


\section{Contents}

Page

1. Introduction . . . . . . . . . . . . . . . . . . 1

2. Theory....................... . . . . 2

2. 1 Thermal Conductivity ............ . 2

2. 2 Electrical Resistivity ........... . 3

2. 3 Lorenz Ratio................ 5

3. Data . . . . . . . . . . . . . . 11

4. Predictive Procedures ............... . . 13

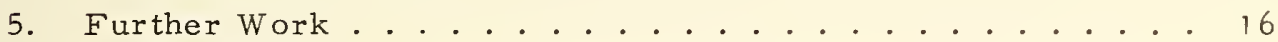

6. Acknowledgments ................... 17

7. References .................... 18

Appendix I Figures and Data Review Tables . . . . . . . . . I-1 Appendix II Data Tabulation ................ II- l Appendix III Bibliography . . . . . . . . . . . . . . . .III-1 
Figure 1. Thermal conductivity of metals and alloys. (a) pure metal (gold), (b) high conductivity alloy (aluminum-7039), and (c) low conductivity alloy (titanium-5Al-2.5 Sn).

Figure 2. Electrical resistivity of metals and alloys. (a) pure metal (gold), (b) high conductivity alloy (aluminum-7039), and (c) low conductivity alloy (titanium-5Al-2.5 Sn).

Figure 3. Electronic Lorenz ratio for pure metals and defect free metals.

Figure 4. Values of $\left(L-L_{0}\right) / L_{0}$ for several classes of commonly used materials.

Figure 5. Values of the total Lorenz number for several classes of commonly used materials.

\section{List of Text Tables}

Table 1. Debye temperatures of selected common metals 


\section{Lorenz Ratios of Technically Important \\ Metals and Alloys}

\section{J. G. Hust and L. L. Sparks}

A comprehensive review and compilation of the world literature on Lorenz ratio of technically important metals and alloys is presented. Lorenz ratio, electrical resistivity, thermal conductivity, and characterization data are compiled in tabular form and the Lorenz ratio data are presented in graphical form as well. Data are included here only if the research reported both thermal conductivity and electrical resistivity of the specimens. No attempt has been made to smooth data or present recommended values.

Key Words: Alloys; compilation; cryogenic; electrical resistivity; Lorenz Ratio; metals; thermal conductivity.

\section{Introduction}

The development of new materials and a renewed interest in existing materials by modern technological industry is creating a demand for thermal and electrical property data. These data are needed for the selection of suitable construction materials and the prediction of operating characteristics of low temperature systems. Apparatus are available at this laboratory as well as others for the accurate measurement of thermal conductivity, electrical resistivity, and thermopower of metals and alloys over the range from 4 to $300 \mathrm{~K}$. However, the measurement of thermal conductivity is slow and expensive; one cannot hope to fill the immediate demand for data by measurement alone. Furthermore, metals and alloys display a wide range in their conductivity values at a given temperature and a single material may exhibit values varying over several orders of magnitude as a function of temperature. One cannot look to theory for these values, since, presently, theoretical predictions represent qualitative guidelines only. However, 
a strong relationship between thermal conductivity and electrical resistivity of metals was observed many years ago and the nature of this correlation has been studied ever since. This relationship, called the Wiedemann-Franz-Lorenz law, makes it possible to predict approximate values of thermal conductivity from less expensive electrical resistivity measurements. For this reas on we feel a compilation of Lorenz ratios of technically important metals is overdue. The predictive value of these data is explained below.

\section{Theory}

In order to better understand the methods of predicting thermal conductivity from the Lorenz ratio, it will be helpful to have an understanding of the general thermal and electrical properties of metals and alloys. The following sections on thermal conductivity and electrical resistivity are included as a review of the fundamental physical behavior.

2. 1 Thermal Conductivity

The thermal conductivity, $\lambda$, of a metal or alloy usually is considered to be the sum of two components- the electronic, $\lambda_{\mathrm{e}}$, and lattice, $\lambda_{\mathrm{g}}$ :

$$
\lambda=\lambda_{e}+\lambda_{g}
$$

There are other mechanisms of heat transport; however, they generally are not applicable here. The lattice term designates the energy carried by the lattice vibrations, called phonons. The subscript g comes from the German word for lattice, Gitter,

In "electrically pure" materials the lattice term is small (usually less than 5 and almost never more than 20 percent of the total) compared to the electron term. Electrical purity is characterized by the residual 
resistivity ratio, $R R R$, between $273 \mathrm{~K}$ and $4 \mathrm{~K}\left(\mathrm{RRR}=\rho_{273} / \rho_{4 \mathrm{~K}}\right)$. The electrical purity of a material is not specified uniquely by the chemical purity of that material; it also depends on the distribution and location of the impurity atoms and on the physical imperfections in the solid. For example, whereas the chemical purity may remain essentially unchanged in annealing, the electrical purity of a material can, in some instances, be changed by an order of magnitude or more. As will be described later, such a change also affects the thermal conductivity.

In alloys, the electronic conductivity is so small, especially at lower temperatures, that the lattice conductivity no longer is negligible. As a matter of fact, $\lambda_{\mathrm{g}}$ is often much larger than $\lambda_{\mathrm{e}}$. The total conductivity of alloys is less than that of pure metals, and some alloys have conductivities approaching those of thermal insulators. The general temperature dependence and relative magnitude of the conductivities of several materials are illustrated in figure 1. More details are given by Powell [1]. 1

\section{2 Electrical Resistivity}

For commercially pure metals and some alloys, the electrical resistivity is described adequately by Matthiessen's rule. This rule, eq(2), states that the electrical resistivity of a metal is the sum of two parts: the intrinsic resistivity, $\rho_{i}$, which is characterized by electrons interacting with phonons only, and the residual resistivity, $\rho_{0}$, which is characterized by electrons interacting with the chemical and physical imperfections of the metal.

$$
\rho(T)=\rho_{0}+\rho_{i}(T)
$$

The residual resistivity is assumed to be temperature independent while

1 Numbers in brackets refer to list of text references at the end of this paper. 


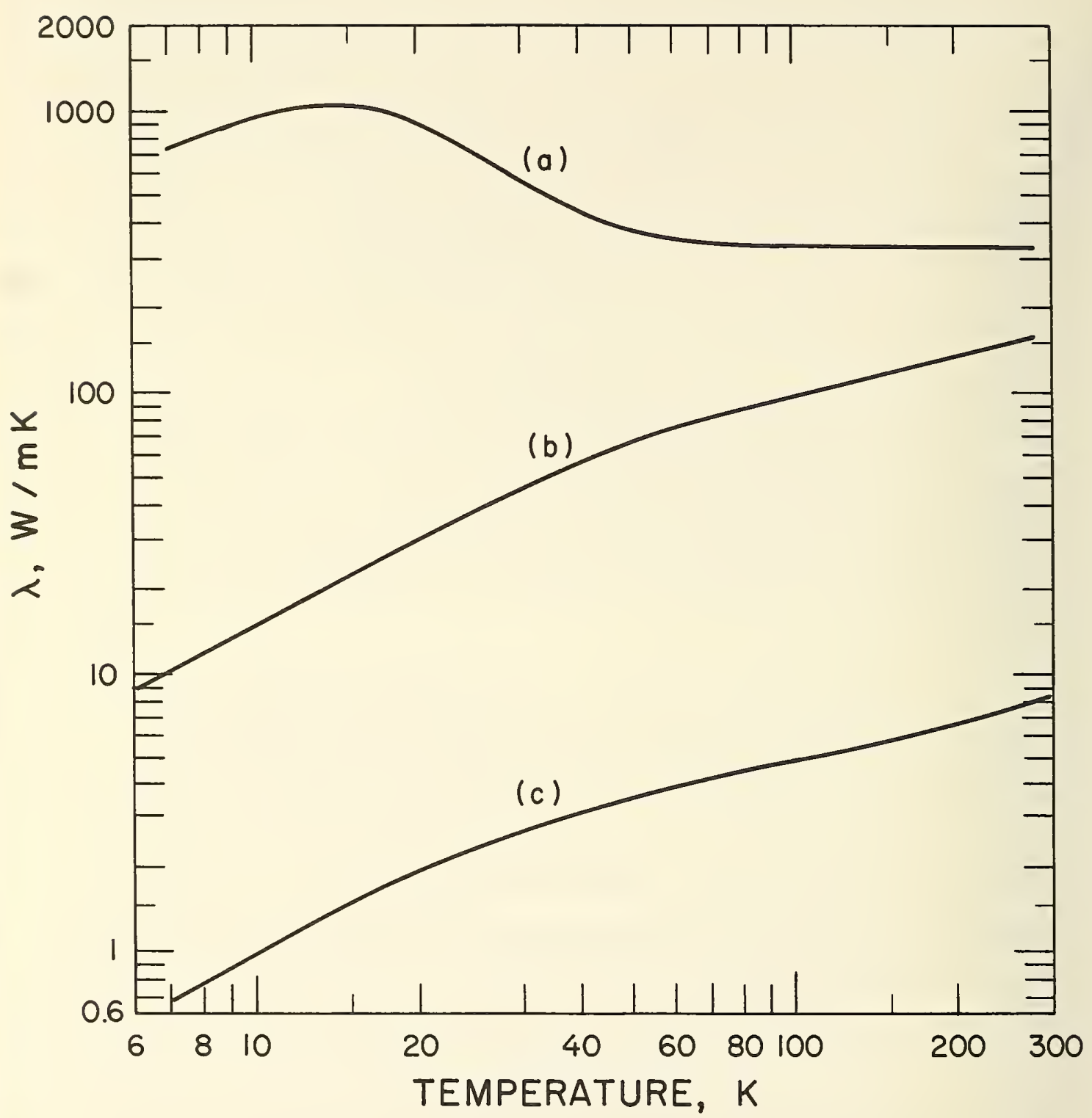

Figure 1. Thermal conductivity of metals and alloys. (a) pure metal (gold), (b) high conductivity alloy (aluminum-7039), and (c) low conductivit alloy (titanium-5A b-2. $5 \mathrm{Sn}$ ). 
the intrinsic resistivity increases rapidly with temperature. The specific temperature dependence varies strongly but systematically both with temperature and material. Nevertheless, $\rho_{i}$ is not dependent significantly upon small composition changes for a given metal or alloy. Thus, if one knows $\rho_{i}(T)$ for a given metal, $\rho(T)$ for different compositions can be obtained from eq(2) after measuring only $\rho_{0}$. The value of $\rho_{0}$ is obtained by measuring $\rho$ at low temperatures where $\rho_{i}$ is negligible (liquid helium temperature is adequate). A few characteristic electrical resistivity curves of pure metals and alloys are given in figure 2.

\section{3 Lorenz Ratio}

In 1853 Wiedemann and Franz formulated an empirical law relating the thermal and electrical conductivities of metals, namely, that the ratio of the electrical and thermal conductivities (WF ratio) at a given temperature is the same for all metals. In 1872 Lorenz discovered that the $\mathrm{W} F$ ratio is proportional to temperature. The result was the WiedemannFranz-Lorenz (W FL) law:

$$
\frac{\lambda}{\sigma}=\lambda \rho=L T \text {, }
$$

where $\sigma=$ electrical conductivity, $L=$ Lorenz number, and $\mathrm{T}=$ absolute temperature.

Drude gave a theoretical derivation of the WFL law for the electronic component of thermal conductivity in 1900 and obtained a value of $2.228 \times$ $10^{-8} \mathrm{~V}^{2} / \mathrm{K}^{2}$ for $\mathrm{L}$. Sommerfeld calculated the first order approximation of $\mathrm{L}$ from the more recent free electron theory of metals. His value, $2.443 \times 10^{-8} \mathrm{~V}^{2} / \mathrm{K}^{2}$, is commonly designated $\mathrm{L}_{\mathrm{O}}$. Electron Lorenz numbers, $\rho \lambda_{e} / T$, other than the Sommerfeld value, will be designated $L_{e}$ to distinguish them from total Lorenz numbers, $L=\rho \lambda / T$. 


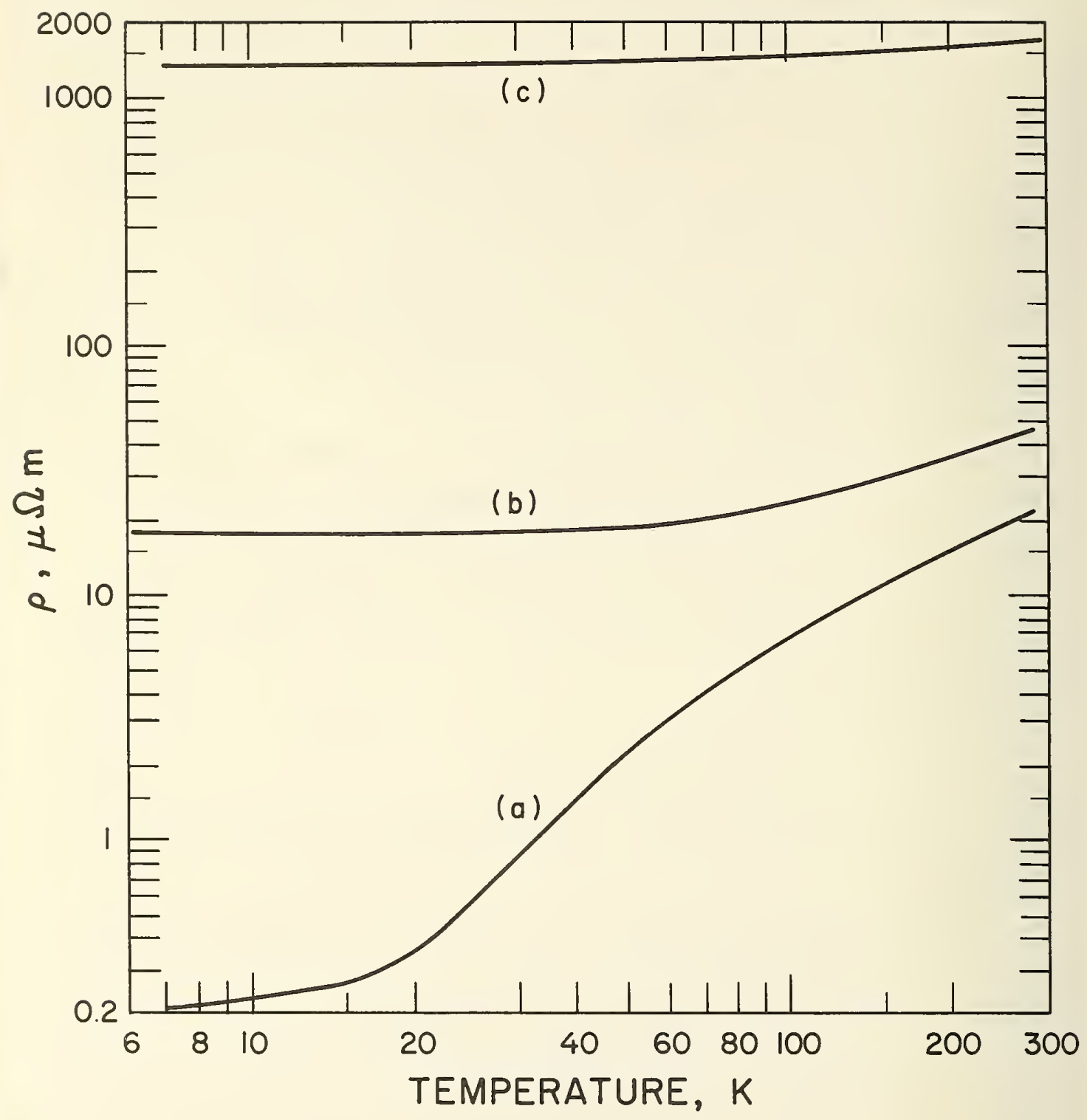

Figure 2. Electrical resistivity of metals and alloys. (a) pure metal (gold), (b) high conductivity alloy (aluminum-7039), and (c) low conductivity alloy (titanium-5A b-2. $5 \mathrm{Sn}$ ). 
For many pure metals the experimentally determined values of $\mathrm{L}$ fall between 2.2 and $3.0 \times 10^{-8} \mathrm{~V}^{2} / \mathrm{K}^{2}$ at room temperature and only slightly higher ( 2 to 3 percent) at $100^{\circ} \mathrm{C}$. At very low temperatures the experimental values of $\mathrm{L}$ are also near the Sommerfeld value. At intermediate temperatures, values of $\mathrm{L}$ below $\mathrm{L}_{\mathrm{O}}$ are observed. The magnitude of the decrease of $\mathrm{L}$ below $\mathrm{L}_{\mathrm{O}}$ increases with increasing purity. The values of $L$ and $L_{e}$ are nearly identical for pure metals since the lattice conductivity is small.

The decrease of $\mathrm{L}_{\mathrm{e}}$ from $\mathrm{L}_{\mathrm{o}}$ in pure metals is caused primarily by the fact that the relaxation times for thermal and electrical conduction processes are different at intermediate temperatures. The electrical conductivity increases with decreasing temperature faster than the thermal conductivity. As the residual resistivity decreases, $\mathrm{L}$ approaches the value for a defect free crystal, thus, resulting in a curve with a lower minimum. The electronic Lorenz ratios of several metals as well as a defect free crystal are shown in figure 3.

For alloys the measured values of Lorenz ratio are always higher than their pure metal counterparts, in some instances considerably higher. However, a similarity still exists in that the $\mathrm{L}$ vs $\mathrm{T}$ curve tends toward $\mathrm{L}_{\mathrm{O}}$ at both extremes of temperature. At intermediate temperatures values of $L$ vary from slightly below $L_{0}$ for dilute alloys to more than ten times larger than $L_{0}$ for some highly alloyed structural metals. A few typical curves for alloys are shown in figures 4 and 5 . The large values of Lorenz ratio observed for these alloys is caused by the presence of a significant lattice conductivity as mentioned previously. Often, knowledge of the magnitude of the lattice conductivity is so limited that it is ignored in the calculation of $L_{e}$ for alloys as it is for pure metals. This is incorrect for alloys, of course, but until $\lambda_{\mathrm{g}}$ is known more accurately, our knowledge of $\mathrm{L}_{\mathrm{e}}$ will also be severely limited. 


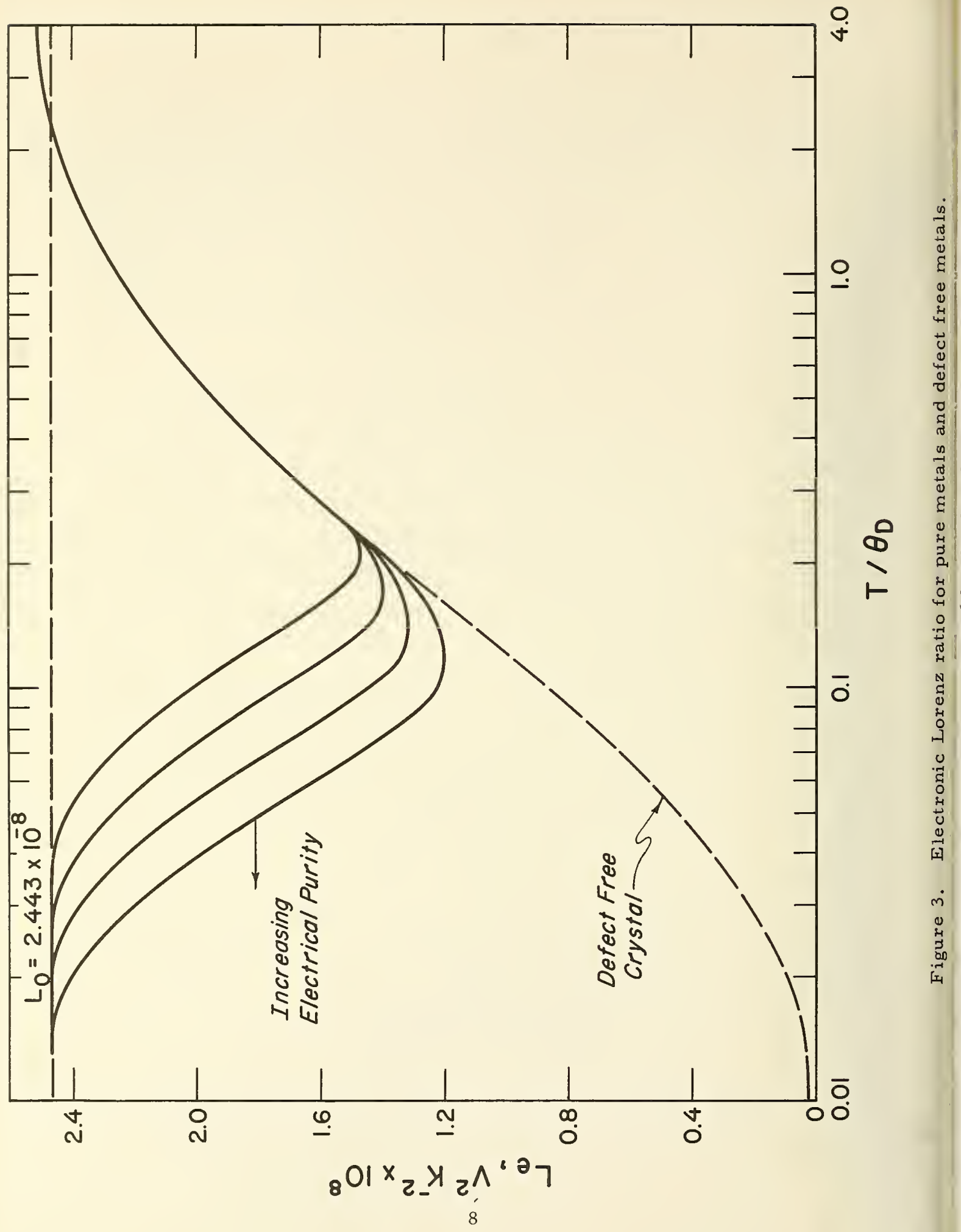




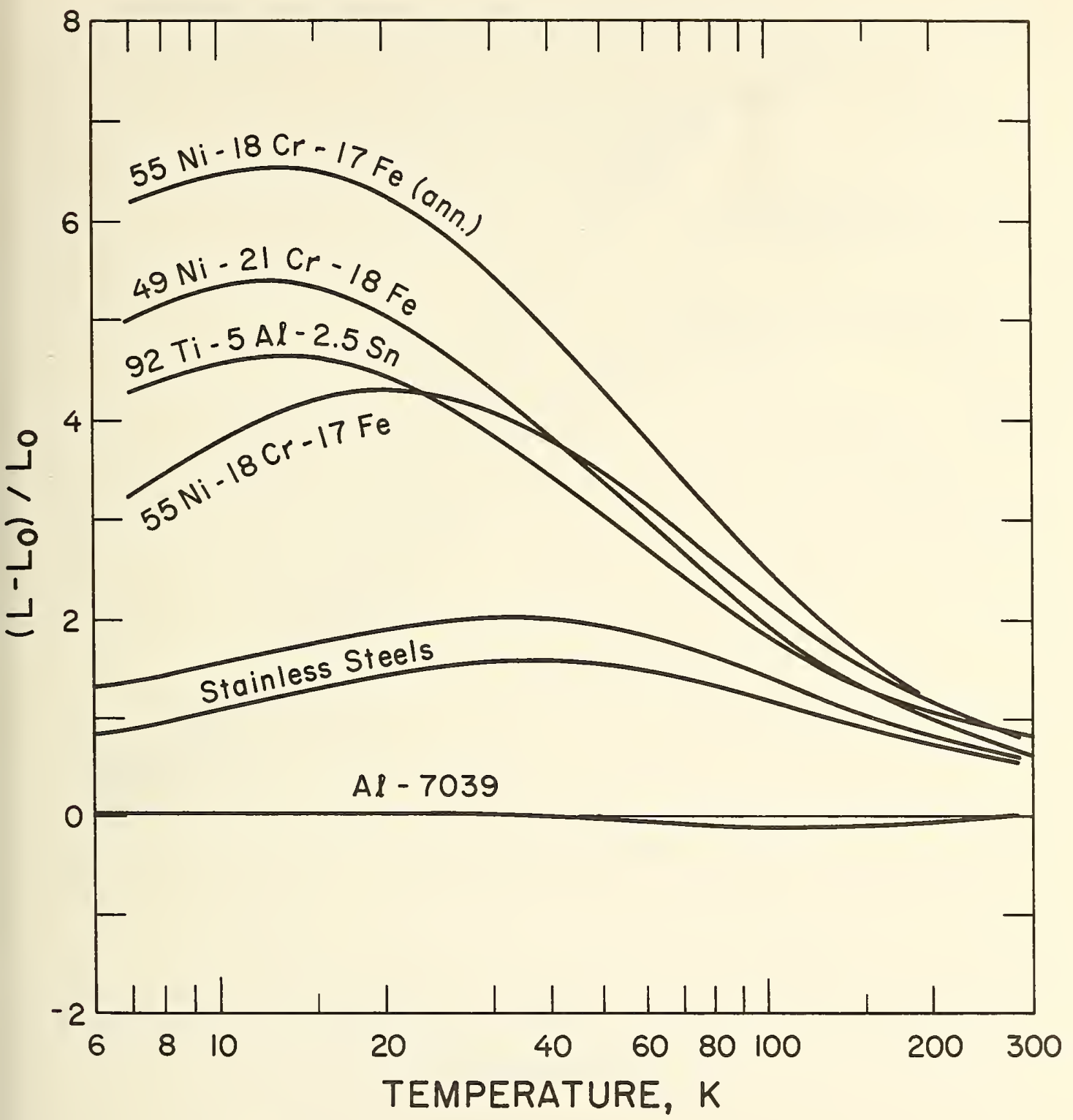

Figure 4. Values of $\left(L-L_{0}\right) / L_{0}$ for several classes of commonly used materials. 


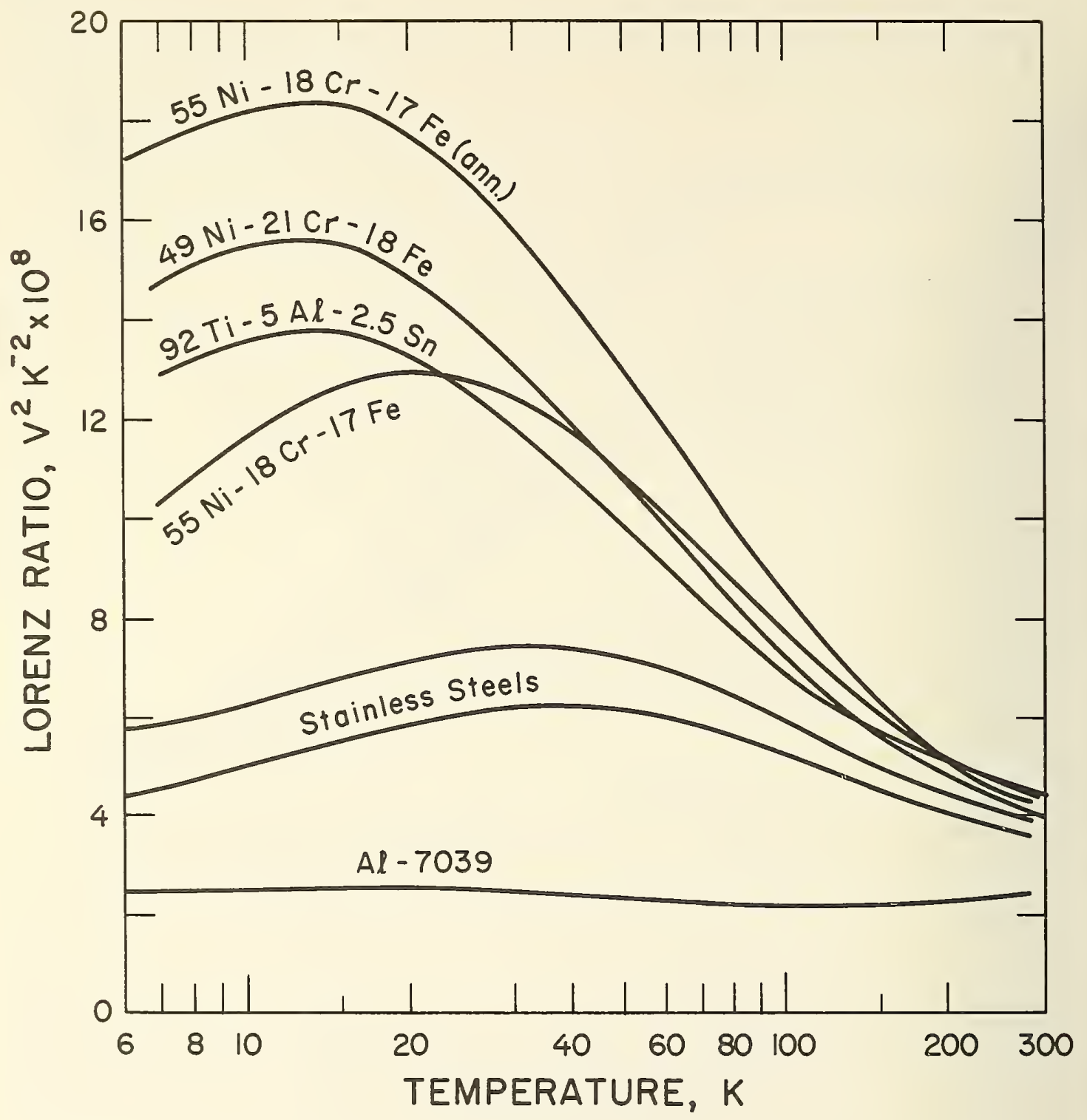

Figure 5. Values of the total Lorenz number for several classes of commonly used materials. 
White [2] recently has compiled values of $L_{e}$ as a function of reduced temperature, $T / \theta_{D}$, for several metals $\left(\theta_{D}\right.$ is the Debye temperature). Values of $\theta_{D}$ for selected metals are shown in table 1 [3]. White's $L_{e}$ data show a variation of only about \pm 2 percent from the mean values to temperatures as low as $0.2 \theta_{\mathrm{D}}$. White calculated $\lambda_{\mathrm{g}}$ from eq (4) below, whose form was proposed by Leibfried and Schlömann [4] among others:

$$
\lambda_{g}=180 \alpha A \theta_{D}^{3} / \gamma^{3} \mathrm{~T} \mathrm{Wm}^{-1} \mathrm{~K}^{-1} \text {. }
$$

In eq (4) $\alpha$ = lattice spacing, $A=$ atomic weight, $\theta_{D}=$ Debye temperature, and $\gamma=$ the Grüneisen parameter. For monovalent metals, values of $\lambda_{\mathrm{g}}$ calculated from eq (4) are only about 2 percent of the total experimentally determined conductivity. For the transition elements iron, platinum, and tungsten, $\lambda_{g}$ values are about 20 percent of the total.

\section{Data}

Childs, et al, [5] of this laboratory have recently made an extensive compilation of thermal conductivities of materials at low temperatures. From their work, publications which contained both electrical and thermal conductivity data for the same materials were identified. These references were searched for data useful to calculate Lorenz ratos and for citations to additional measurements. This search resulted in the reference sources listed in Appendix III. The electrical resistivity and thermal conductivity data from these reference sources were extracted and converted to a common set of units (SI). Lorenz ratios were calculated and are plotted in the figures of Appendix I. The tabular values of electrical resistivity, thermal conductivity, and Lorenz ratios as reported in the literature are given in the right hand columns of Appendix II. The computed values of Lorenz ratio in SI units are given in the left hand columns. 
TABLE 1. Debye temperatures of selected common metals

[3]

Substance

Aluminum

Beryllium

Chromium

Copper

Gold

Iron

Lead

Magnesium

Manganese

Molybdenum

Nickel

Platinum

Silver

Tantalum

$\mathrm{T}$ in

Titanium

Tungsten

Zinc
Debye temperature, $\theta_{D}$

(K)

426

1160

610

344

165

464

96

406

476

440

440

240

225

250

195

420

405

300 
In many instances, data had to be read from small graphs. The error introduced by reading these graphs was sometimes above $10 \%$. This is unfortunate especially in those instances where the uncertainty of the original experimental data was smaller than 10\%. In some instances where data are closely spaced, curves were drawn to represent these data. No attempt was made to smooth the data or to present best values. Considerably more work, both experimental and theoretical, must be done before smoothing can be done with confidence.

It is difficult to assess the uncertainty of thermal conductivity data. Probably the best one should expect is about $1 \%$ and the worst (except for blunders) is about $50 \%$. Many sources list uncertainties between 5 and $10 \%$. The Lorenz ratios will have comparable uncertainties since the uncertainty in electrical resistivity is generally much smaller. Childs, et $\mathrm{al},[5]$ made estimates of thermal conductivity uncertainty for each of the references. These estimates are not repeated here.

\section{Predictive Procedures}

In order to use the Lorenz ratio data presented here in predicting thermal conductivities of new materials one must associate this material with one whose $\mathrm{L}(\mathrm{T})$ is already known and measure the electrical resistivity of the new material. Then one may compute

$$
\lambda=\frac{L T}{\rho} .
$$

It is stressed that obtaining $\rho$ is significantly easier than measuring $\lambda$. The association of one material with another may be done primarily from a knowledge of its composition but also from other characterization parameters such as electrical resistivity, hardness, density, or crystalline structure. The thermal and mechanical histories of the specimen are important as well. 
In the event that no similar material has been measured, generally less accurate techniques of prediction may be considered. For purposes of prediction, divide materials into the following classes:

(1) pure metals,

(2) high conductivity alloys,

(3) low conductivity alloys,

and consider temperatures in three ranges:
(a) $\mathrm{T}>\theta_{\mathrm{D}}$,
(b) $\mathrm{T}<\theta_{\mathrm{D}}$,
(c) $\mathrm{T} \ll \theta_{\mathrm{D}}$.

We will consider pure metals first. At temperatures above $\theta_{D}$ or much less than $\theta_{D}$ (say $\left.0.01 \theta_{D}\right)$, one can make reasonable estimates of thermal conductivity of pure metals by calculating $\lambda_{e}$ as $L_{0} T / \rho$. The lattice conductivity, $\lambda_{\mathrm{g}}$, is computed from eq (4)and the total conductivity $\lambda$, from eq(1). The contribution of the lattice conductivity for pure metals is usually negligible. The electrical resistivity, $\rho(T)$, can often be obtained directly from the literature $[6,7]$ or from a measurement of $\rho_{0}$ and values of $\rho_{i}(T)$, also found in the literature. At temperatures from about $0.2 \theta_{\mathrm{D}}$ to $\theta_{\mathrm{D}}, \mathrm{L}$ and $\mathrm{L}_{\mathrm{e}}$ are temperature dependent but not appreciably dependent on purity. Thus, one can use the $L_{e}$ curve obtained by White [2], shown in figure 3, to calculate $\lambda_{e}$ from $L_{e} T / \rho$. Between $0.01 \theta_{D}$ and $0.2 \theta_{D}$, the electronic and total Lorenz numbers are dependent on purity and temperature. No correlation between $\mathrm{L}$ and purity has been mathematically formulated, although it is clear that one exists. It is noted that at temperatures above about $0.2 \theta_{\mathrm{D}}$, the thermal conductivity of pure metals is not strongly dependent upon the chemical or physical condition of the metal. Thus, if accurate conductivity values have been obtained for a pure metal, the se values above $0.2 \theta_{\mathrm{D}}$ are applicable to other specimens to within about $10 \%$. 
Next we will consider high conductivity alloys. High conductivity alloys are generally dilute alloys but may also be the result of alloying similar elements. Ordered alloys also have high conductivities. The values of $\mathrm{L}$ for high conductivity alloys are generally near $\mathrm{L}_{0}$ at all temperatures. Thus, a first approximation of thermal conductivity can be obtained from $L_{0} T / P$.

Last we will consider the low conductivity alloys. Generally, the se are highly alloyed structural alloys. Prediction of the thermal conductivity is most difficult at temperatures $T \leq \theta_{D}$ in the low conductivity alloys. Here the lattice conductivity is comparable to and sometimes much larger than the electron conductivity. If a comparable alloy has not been measured, it is probably impossible to predict a reasonable accurate value of $\lambda$ for this case. We presently are engaged in a program to improve the predictive capabilities for such alloys. It appears reasonably obvious that to do this one must be able to calculate the electron component from the electrical resistivity and the lattice component by some other means. The lattice component probably will be obtained from a modified form of eq (4), but additional research will be required before a more accurate equation is found and its limitations are understood. At temperatures above $\theta_{D}$, one can obtain a first approximation of thermal conductivity, usually on the low side, from $L_{0} T / \rho$. The above methods of prediction are summarized as follows: 


\begin{tabular}{|c|c|c|c|}
\hline & $\begin{array}{l}\text { Pure } \\
\text { Metals }\end{array}$ & $\begin{array}{c}\text { High Conductivity } \\
\text { Alloys }\end{array}$ & $\begin{array}{c}\text { Low Conductivity } \\
\text { Alloys }\end{array}$ \\
\hline$T \ll \theta_{D}$ & $\lambda \approx L_{0} \mathrm{~T} / \mathrm{p}$ & $\lambda \approx L_{0} T / \rho$ & No \\
\hline$T<\theta_{D}$ & 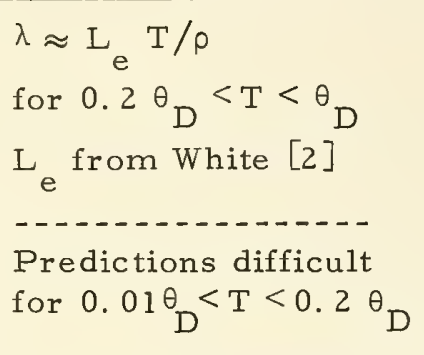 & $\lambda \approx L_{0} T / \rho$ & $\begin{array}{c}\text { Palid } \\
\text { Predicting } \\
\text { Method } \\
\text { Known }\end{array}$ \\
\hline$T>\theta_{D}$ & $\lambda \approx \mathrm{L}_{\mathrm{o}} \mathrm{T} / \mathrm{\rho}$ & $\lambda \approx L_{0} \mathrm{~T} / \mathrm{\rho}$ & $\lambda \approx L_{0} T / \rho_{0}$ \\
\hline
\end{tabular}

5. Further Work

It is seen from this compilation that the Lorenz ratios of pure metals are near the Sommerfeld value, $L_{0}=2.443 \times 10^{-8} \mathrm{~V}^{-2} / \mathrm{K}^{2}$, at low temperatures (residual region), fall to lower values at intermediate cryogenic temperatures, and increase again to near $L_{0}$ at high temperatures. At intermediate temperatures a dependence on impurity concentration is observed. For alloys one obtains the expected increase in Lorenz ratio at intermediate temperatures instead of a dip as for the pure metals. This increase in $\mathrm{L}$ is evidence of a relatively large lattice conductivity. Somewhat challenging from a predictive standpoint is the large spread in Lorenz ratios for the given classes of materials at each temperature. This large spread will result in large uncertainties in predicted thermal conductivities based on these curves. Further subdivision of materials may be necessary to achieve a sufficiently small spread to be useful.

The objective of this Lorenz ratio compilation is to separate materials into classes based on similar Lorenz ratio curves. From 
these curves and a knowledge of the electrical resistivity of a new material from that class, it is often possible to approximate the thermal conductivity of the new material. This compilation has revealed some other interesting possibilities for prediction of thermal conductivities. For example, in several instances data exist in the literature which will allow computation of the specific thermal resistivity resulting from controlled addition of impurity atoms in a given host material. It has been noted that some impurities are much more effective scatterers than others. From a table of specific thermal resistivities, similar to that compiled by Blatt [8] for electrical resistivity, one may be able to accurately predict the electronic thermal conductivity of metals and alloys. Of the huge number of host-impurity element combinations which exist, only a few have been measured. However, there is a good possibility that one can construct such a table from a knowledge of $L(T)$ for pure metals and Blatt's specific electrical resistivities. The measurements which have been compiled here and by Childs, et al., [5] can be used to check the accuracy of such calculations. The available data will also be useful in examining lattice conductivity.

\section{Acknowledgments}

The authors express appreciation to the many people who contributed to make this work possible. Most of the program was supported by NASA (SNSO-C) Contract R-45. Steve Schmidt and Mark Prouhet assisted in data extracted from the many sources. Gregg Childs and Bob Powell were very helpful in providing a bibliography, as yet unpublished, from their thermal conductivity compilation as well as opening their reference files for our use. 


\section{References}

[1] Powell, R. L., "Thermophysical Properties of Metals at Cryogenic Temperatures, "Behavior of Metals at Cryogenic Temperatures, A.STM STP 387, pp. 134-148, American Society for Testing and Materials (1966).

[2] White, G. K. , "Lattice Conductivity and Lorenz Ratio of Standard Metals, " 8th Thermal Conductivity Conference, C. Y. Ho and R. E. Taylor, eds., pp. 37-44, Plenum Press, New York (1969).

[3] Corruccini, R. J., and Gniewek, J. J., Nat. Bur. of Stand. (U. S. ), Monogr. 21, 20 pages (1960).

[4] Leibfried, G., and Schlömann, E., Nachrichten der Akademie der Wissenschaften in Gottingen, Vol. II-A, No. 4, pp. $71-93$ (1954).

[5] Childs, G. E., Ericks, L. J., and Powell, R. L., Thermal Conductivity of Solids at Room Temperature and Below, A Review Compilation of the Literature, Nat. Bur. of Stand. (U. S. ), Monogr. 131 (1973).

[6] Clark, A. F., Childs, G. E., and Wallace, G. H., Cryogenics, 10, 295-305 (1970).

[7] Meaden, G. T., Electrical Resistance of Metals, Plenum Press, New York (1965).

[8] Blatt, F. J., Physics of Electronic Conduction in Solids, McGrawHill Book Company N. Y. p. 199 (1968). 


\section{APPENDIX I - FIGURES \\ AND \\ DATA REVIEW TABLES}

The use in this paper of trade names is essential to a proper understanding of the work presented. Their use in no way implies any approval, endorsement, or recommendation by NBS. 
Figure 1 Lorenz ratio of beryllium . . . . . . . . . . . I- 5

Figure 2 Lorenz ratio of aluminum . . . . . . . . . . . I- 9

Figure 3 Lorenz ratio of aluminum alloys . . . . . . . . . I- 11

Figure 4 Lorenz ratio of lead and tin . . . . . . . . . . I-13

Figure 5 Lorenz ratio of gold . . . . . . . . . . . . . I-15

Figure 6 Lorenz ratio of gold-cobalt alloy . . . . . . . . . I-17

Figure 7 Lorenz ratio of silver . . . . . . . . . . . . . . . I- 19

Figure 8 Lorenz ratio of copper . . . . . . . . . . . . . I-21

Figure 9 Lorenz ratio of copper alloys (German silver and brass) ................... . . I-24

Figure 10 Lorenz ratio of zinc and cadmium . . . . . . . . I-26

Figure 11 Lorenz ratio of scandium and yttrium . . . . . . I-28

Figure 12 Lorenz ratio of titanium, hafnium, and zirconium ................. . . . . . . 30

Figure 13 Lorenz ratio of titanium alloys . . . . . . . . . . . I-32

Figure 14 Lorenz ratio of tungsten. . . . . . . . . . . . . . I-34

Figure 15 Lorenz ratio of molybdenum . . . . . . . . . . . I-36

Figure 16 Lorenz ratio of chromium. . . . . . . . . . . . I-38

Figure 17 Lorenz ratio of manganese and rhenium . . . . . .I-40

Figure 18 Lorenz ratio of iron . . . . . . . . . . . . . . . I-43

Figure 19 Lorenz ratio of stainless and alloy steels . . . . . I-47

Figure 20 Lorenz ratio of carbon steels . . . . . . . . . . . I-49

Figure 21 Lorenz ratio of cobalt ................ . I-51

Figure 22 Lorenz ratio of nickel . . . . . . . . . . . . I-53

Figure 23 Lorenz ratio of nickel alloys . . . . . . . . . . . . I-55

Figure 24 Lorenz ratio of platinum . . . . . . . . . . . . I-57

Figure 25 Lorenz ratio of palladium . . . . . . . . . . . I-59 
Beryllium ...................... I- 4 Magnesium ................... . I- 6 Aluminum .................... . I- 7 Aluminum Alloys ................. I- 10 Lead and Tin ................ I- 12 Gold ...................... I- 14

Gold Cobalt Alloy ................. I- 16 Silver .................... I- 18 Copper.................... . . I- 20 Copper Alloys (German Silver and Brass) . . . . . I-22 Zinc and Cadmium ............... I- 25 Scandium and Yttrium . . . . . . . . . . I- 27 Titanium, Hafnium, and Zirconium ........ I-29 Titanium Alloys. ............... I-31 Tungsten. . . . . . . . . . . . . . I- 33

Molybdenum . . . . . . . . . . . . . . I- 35 Chromium ................. I I-37 Manganese and Rhenium ............. . I- 39 Iron .................. I- 41 Stainless and Alloy Steels. . . . . . . . . . . I- 44 Carbon Steels................ I- . . 48 Cobalt................... I- 50 Nickel .................. I- 52 Nickel Alloys .. . . . . . . . . . . . . I- 54 Platinum................ I- 56 Palladium ................ . . I- 58 


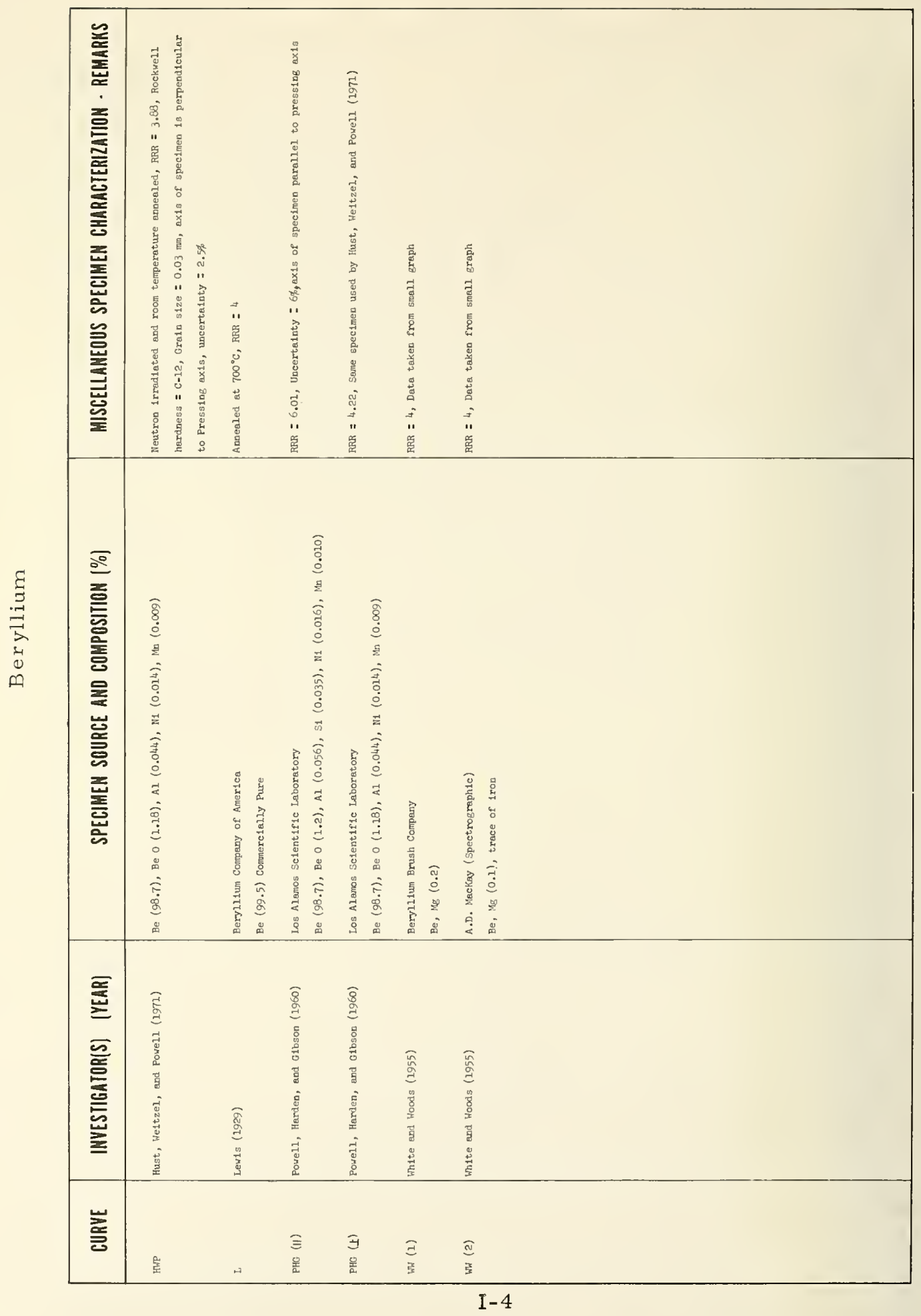




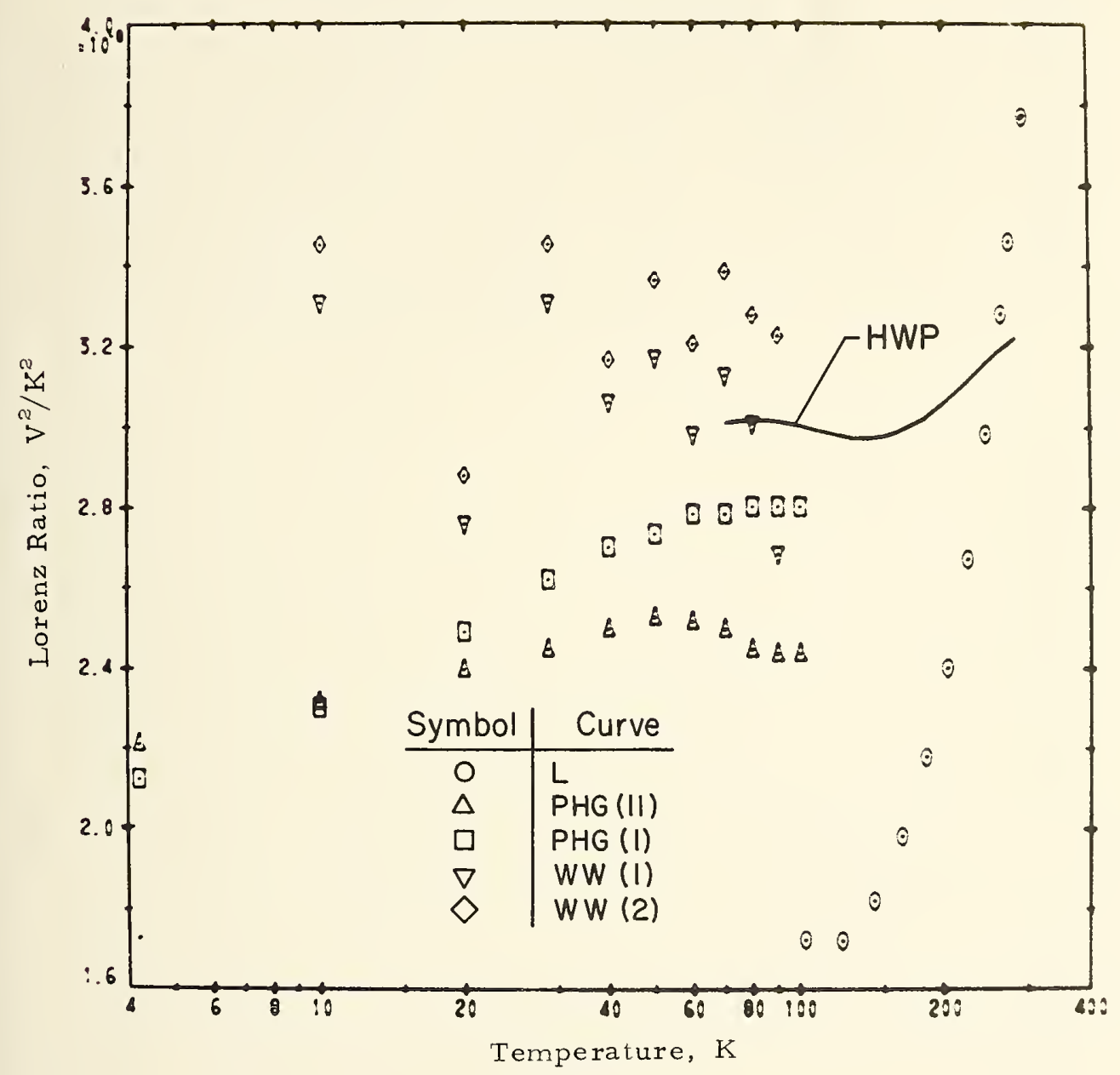

Figure 1. Lorenz ratio of beryllium 


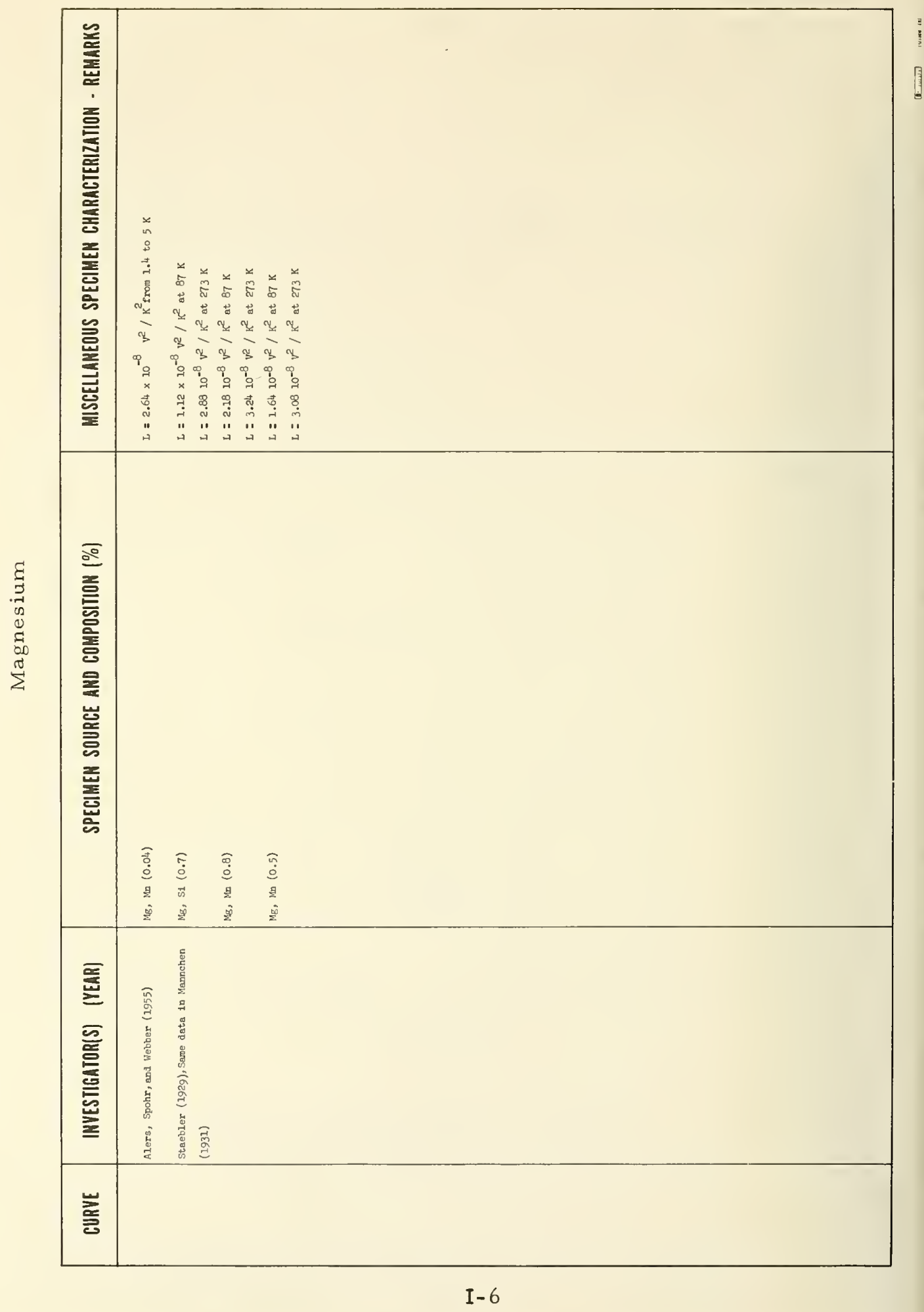




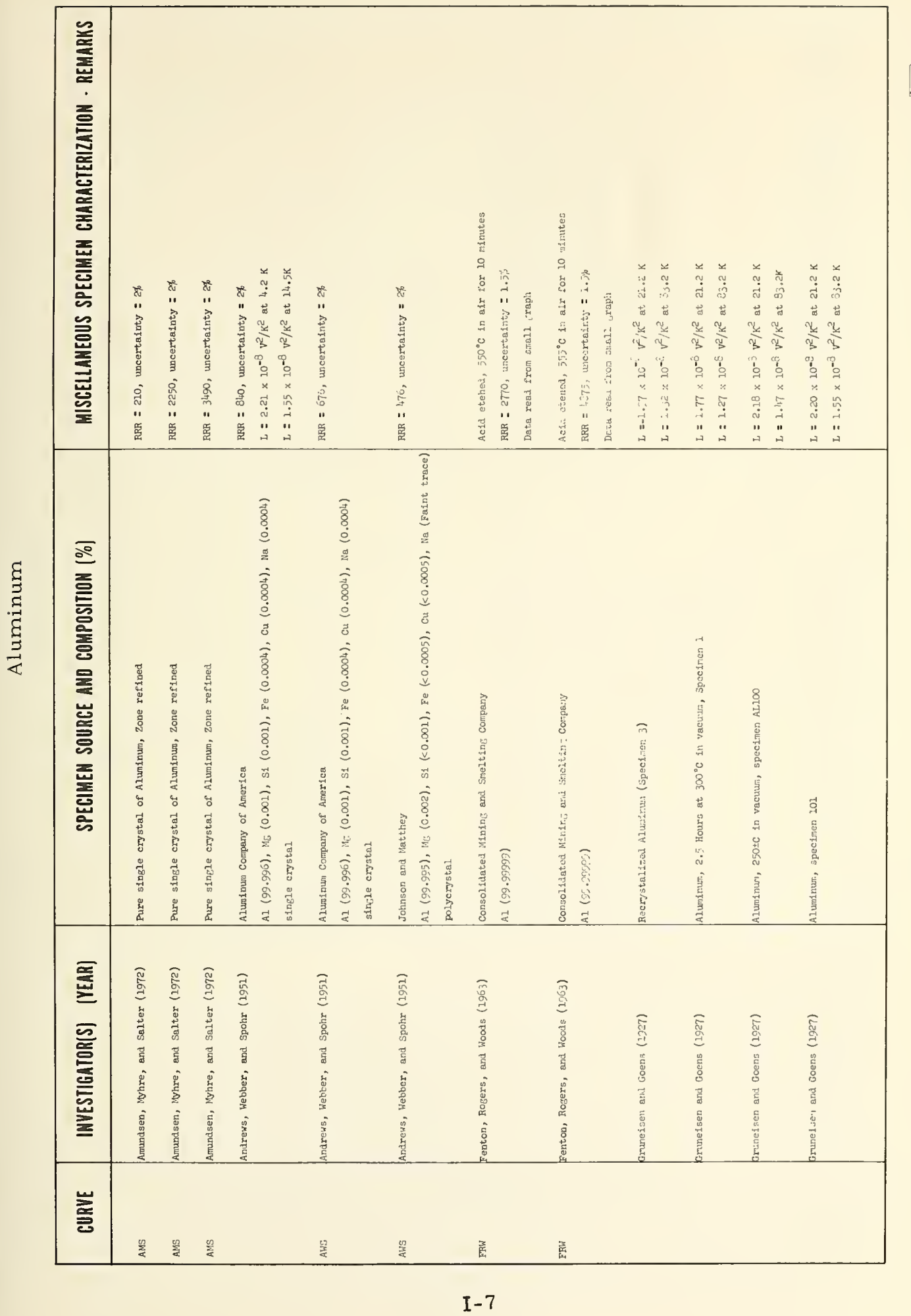




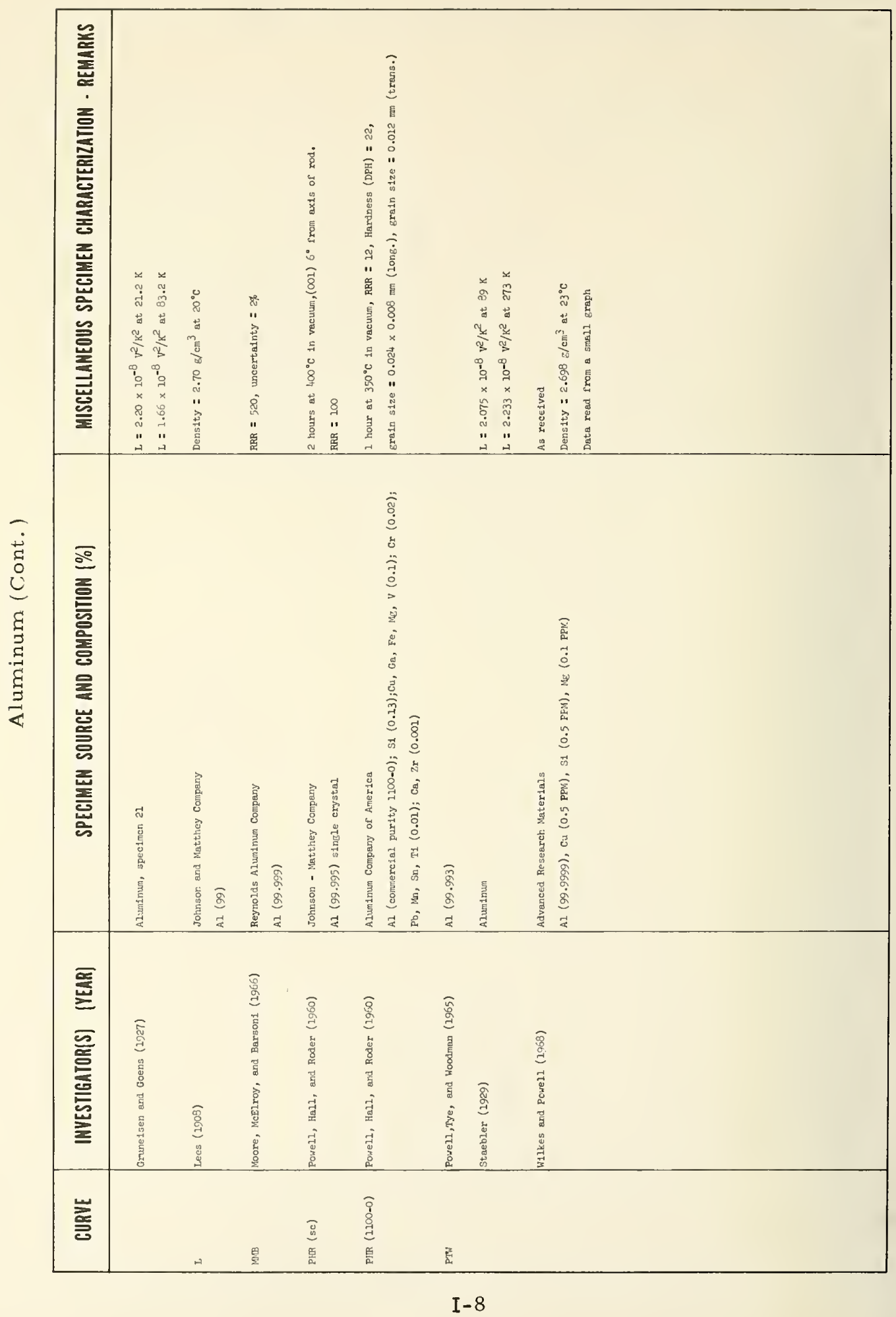




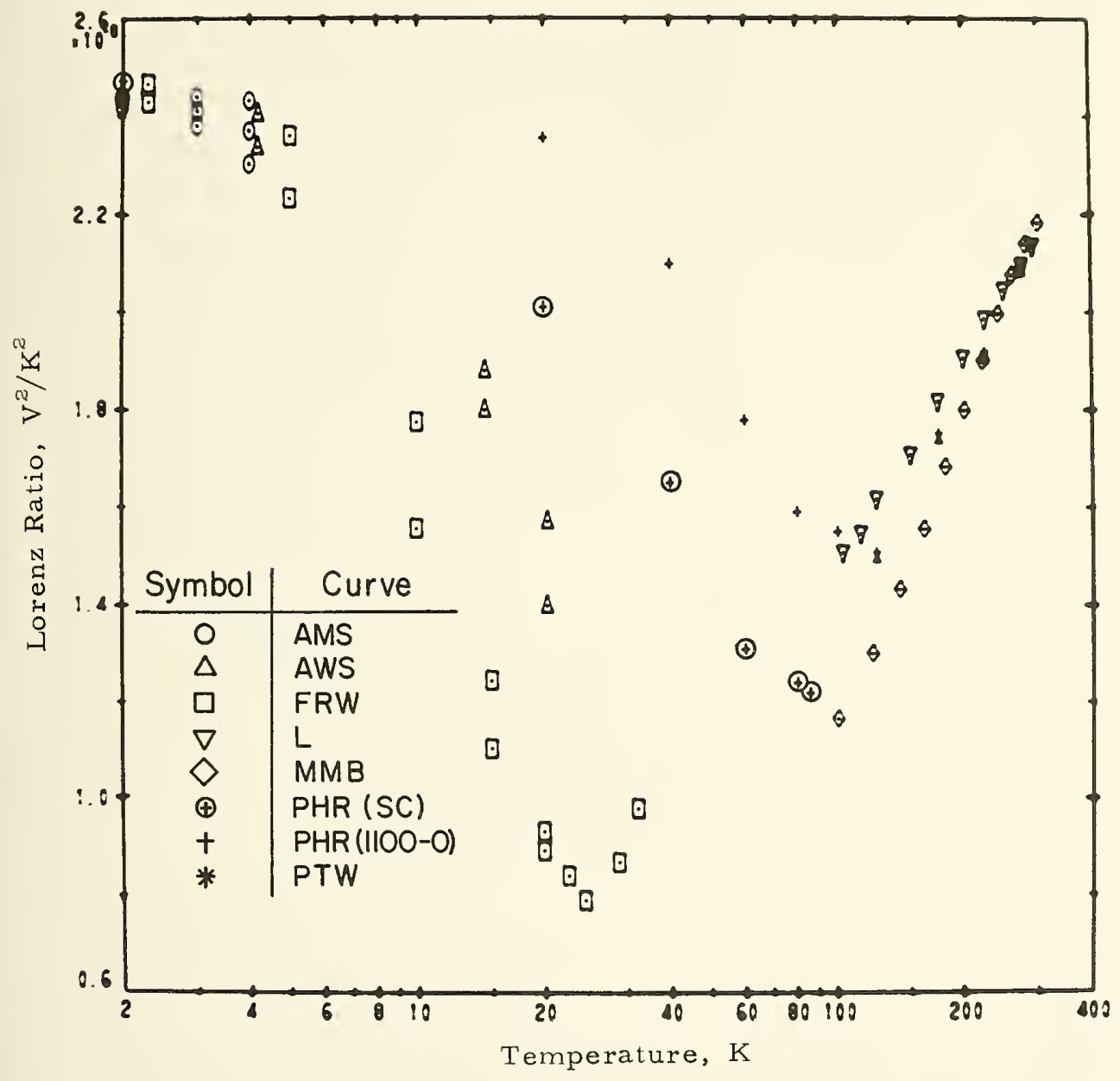

Figure 2. Lorenz ratio of aluminum 


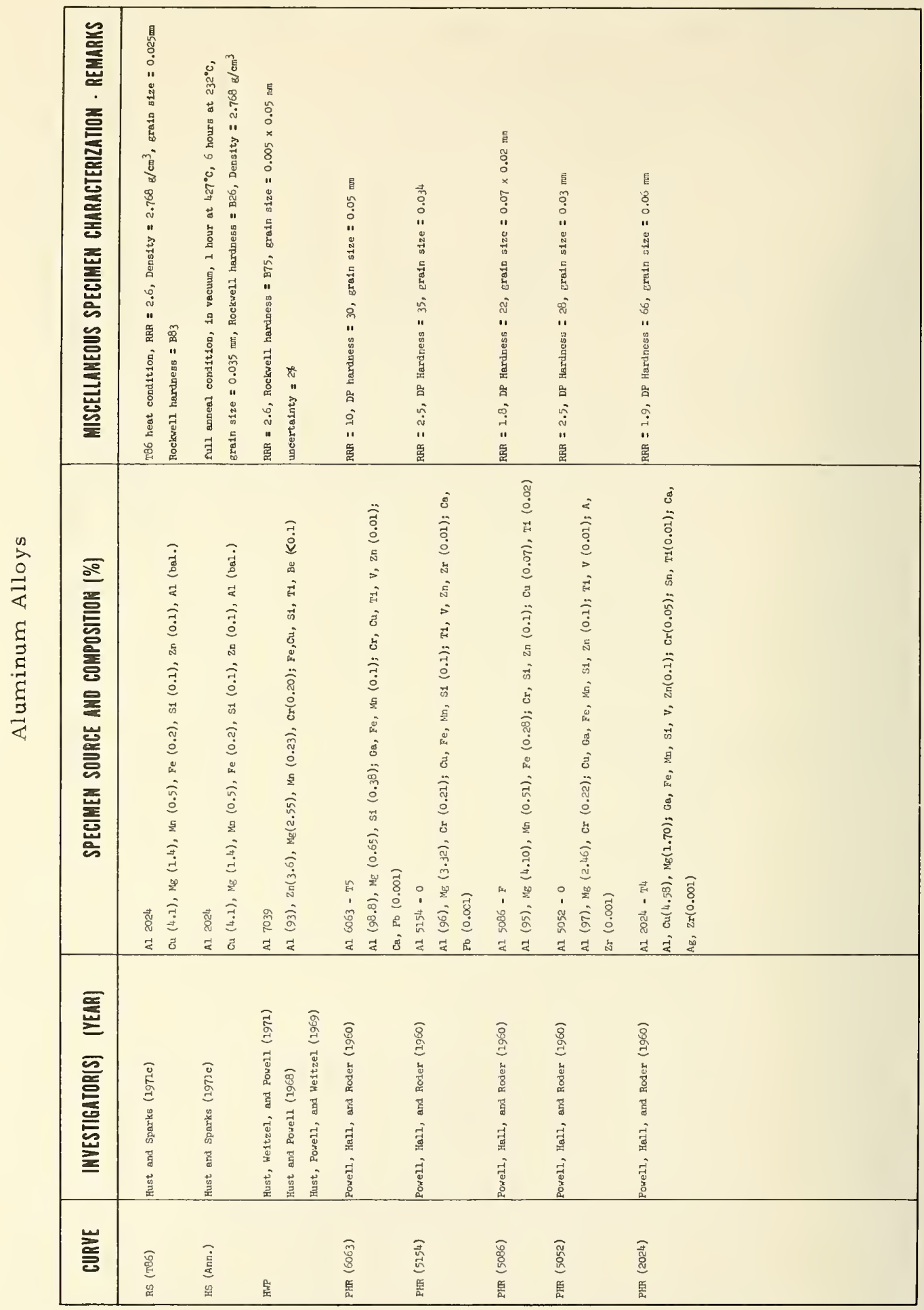




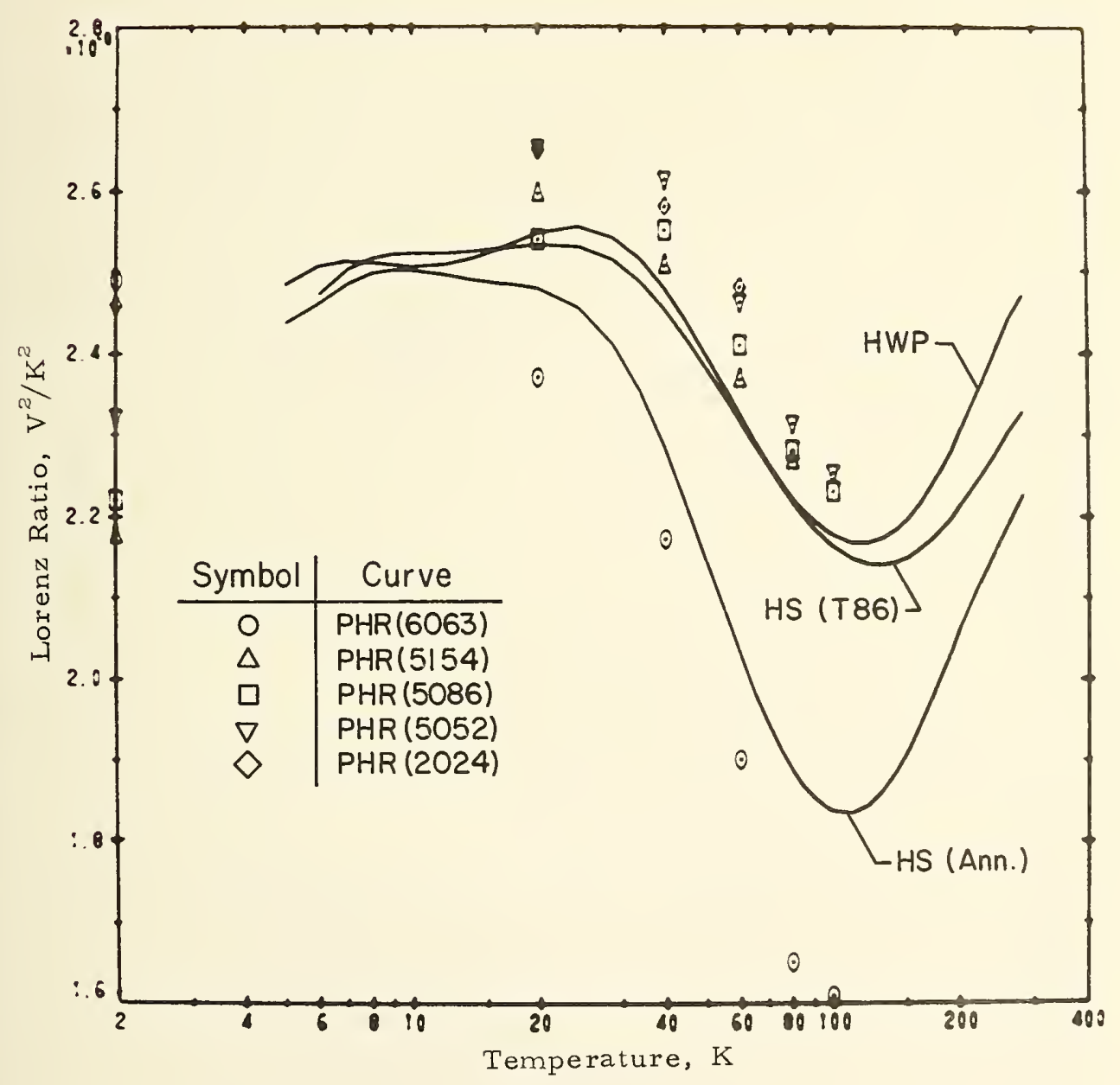

Figure 3. Lorenz ratio of aluminum alloys 


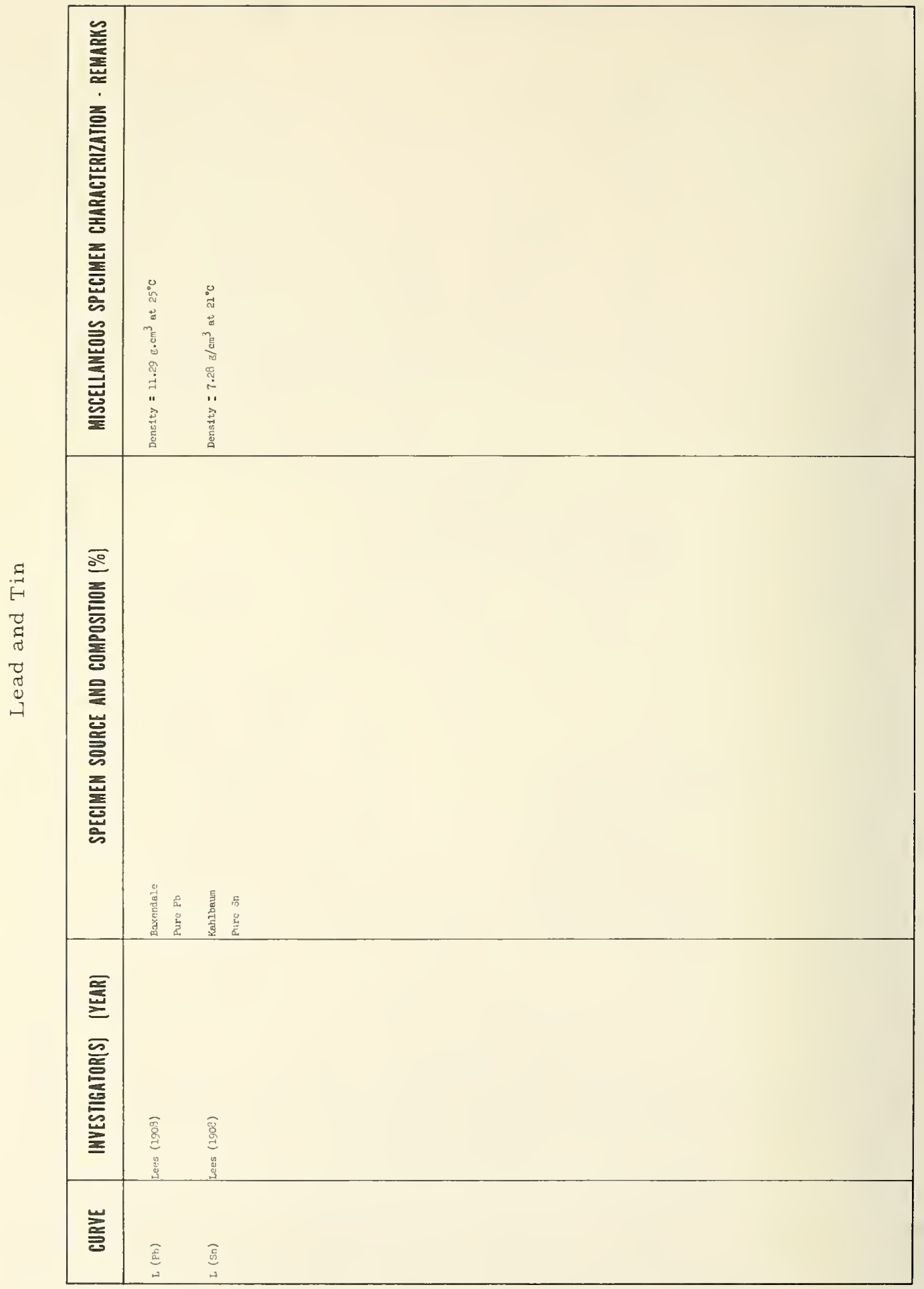

I- 12 


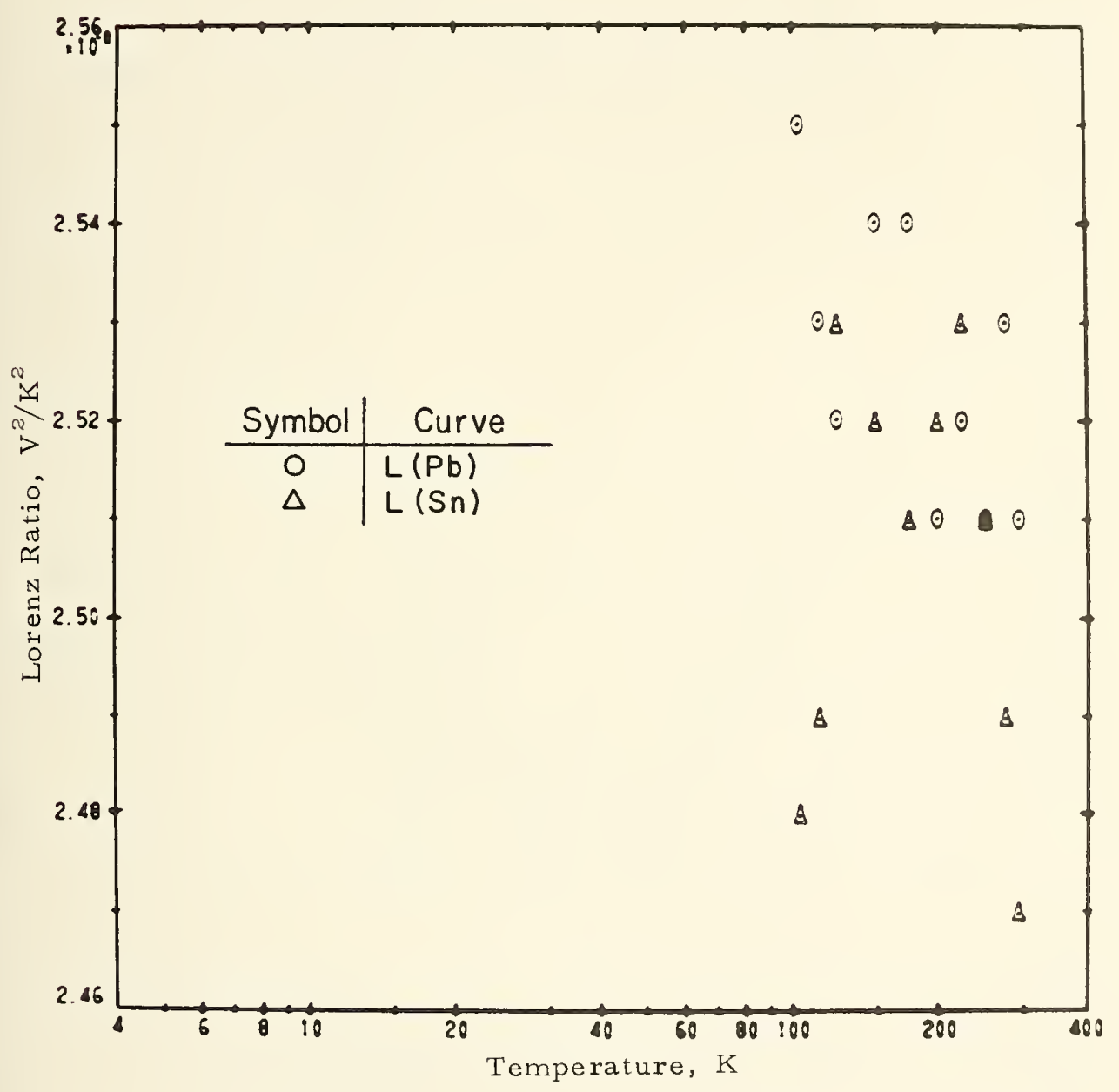

Figure 4. Lorenz ratio of lead and tin 


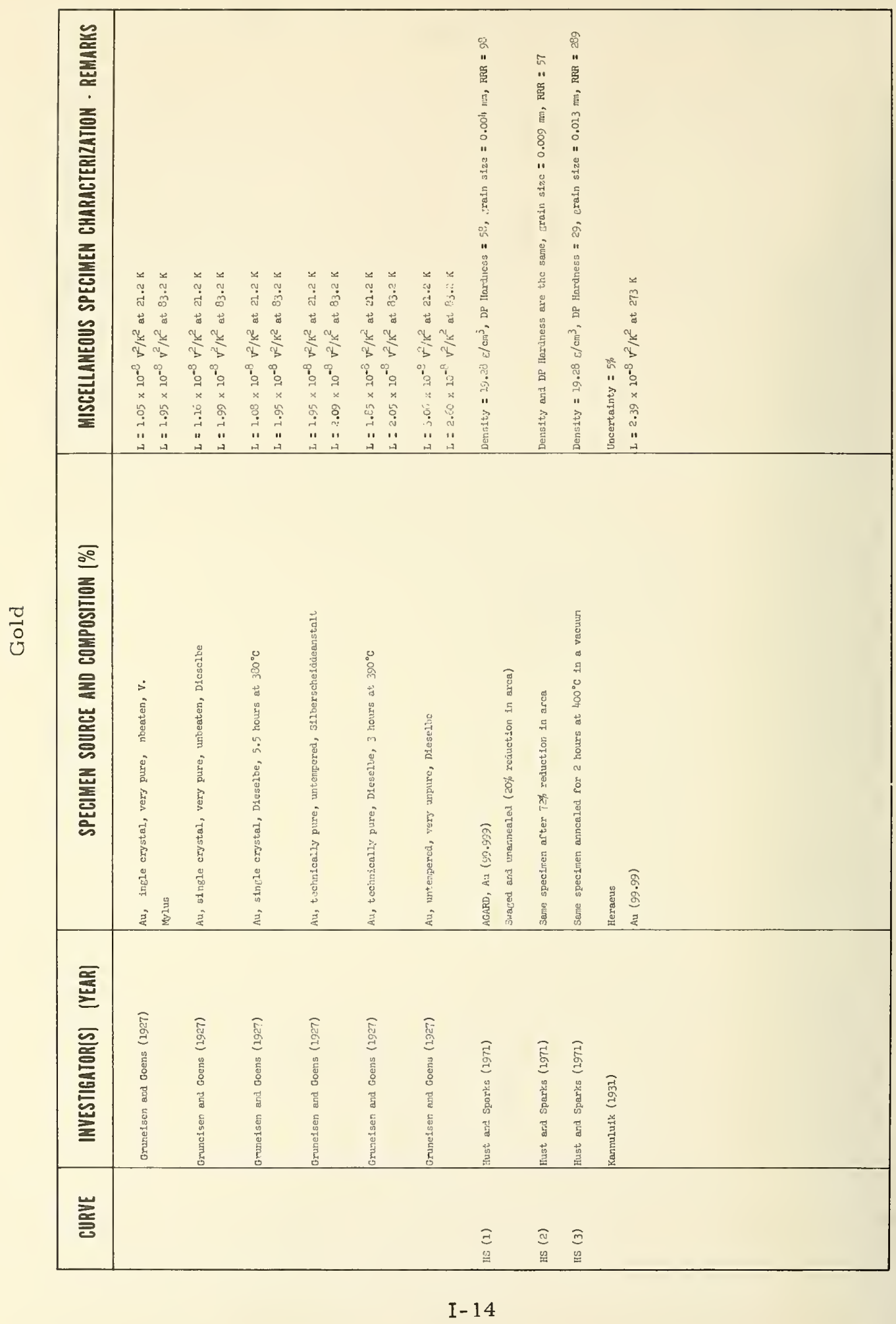




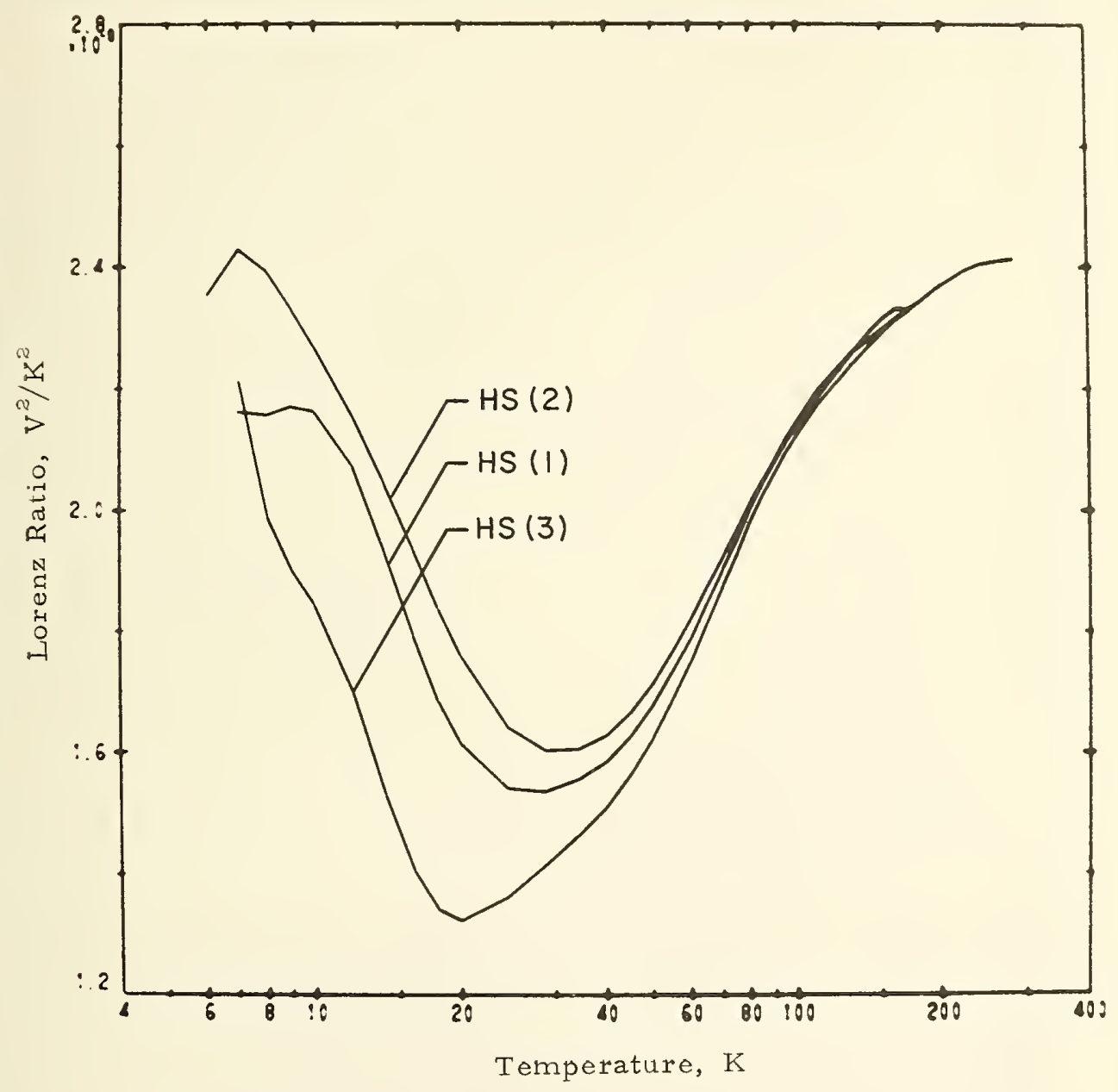

Figure 5. Lorenz ratio of gold 


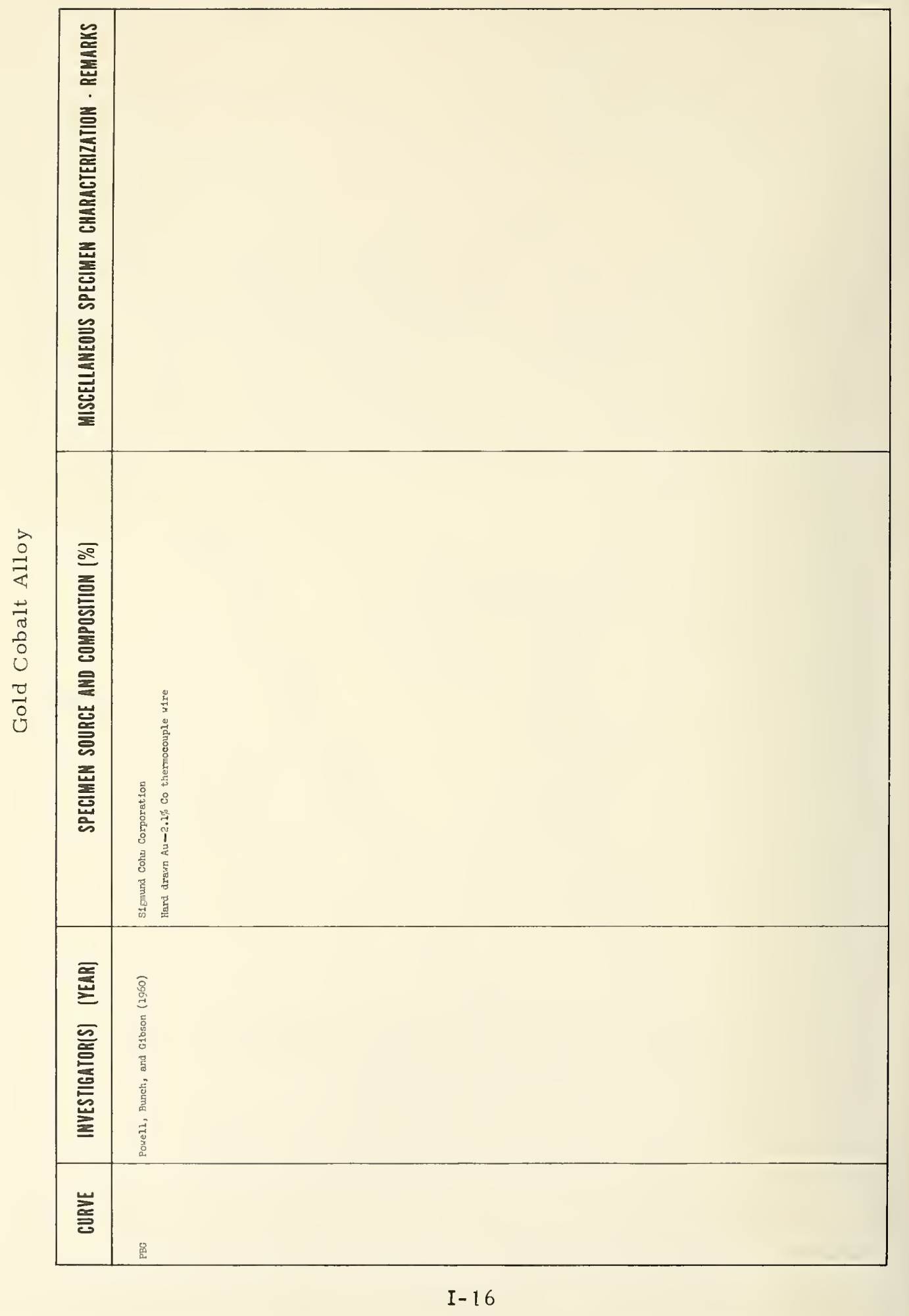




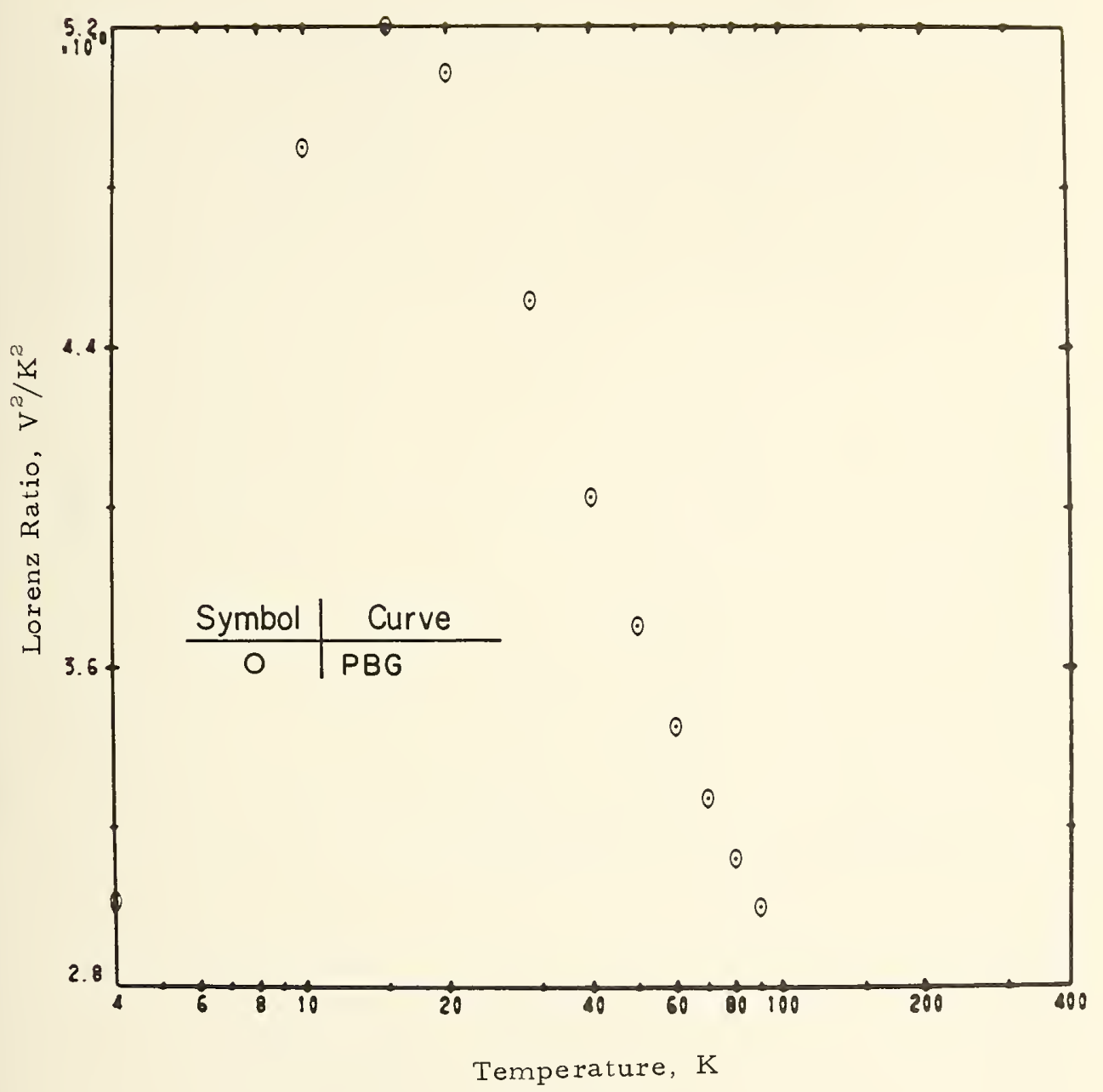

Figure 6. Lorenz ratio of gold-cobalt alloy 


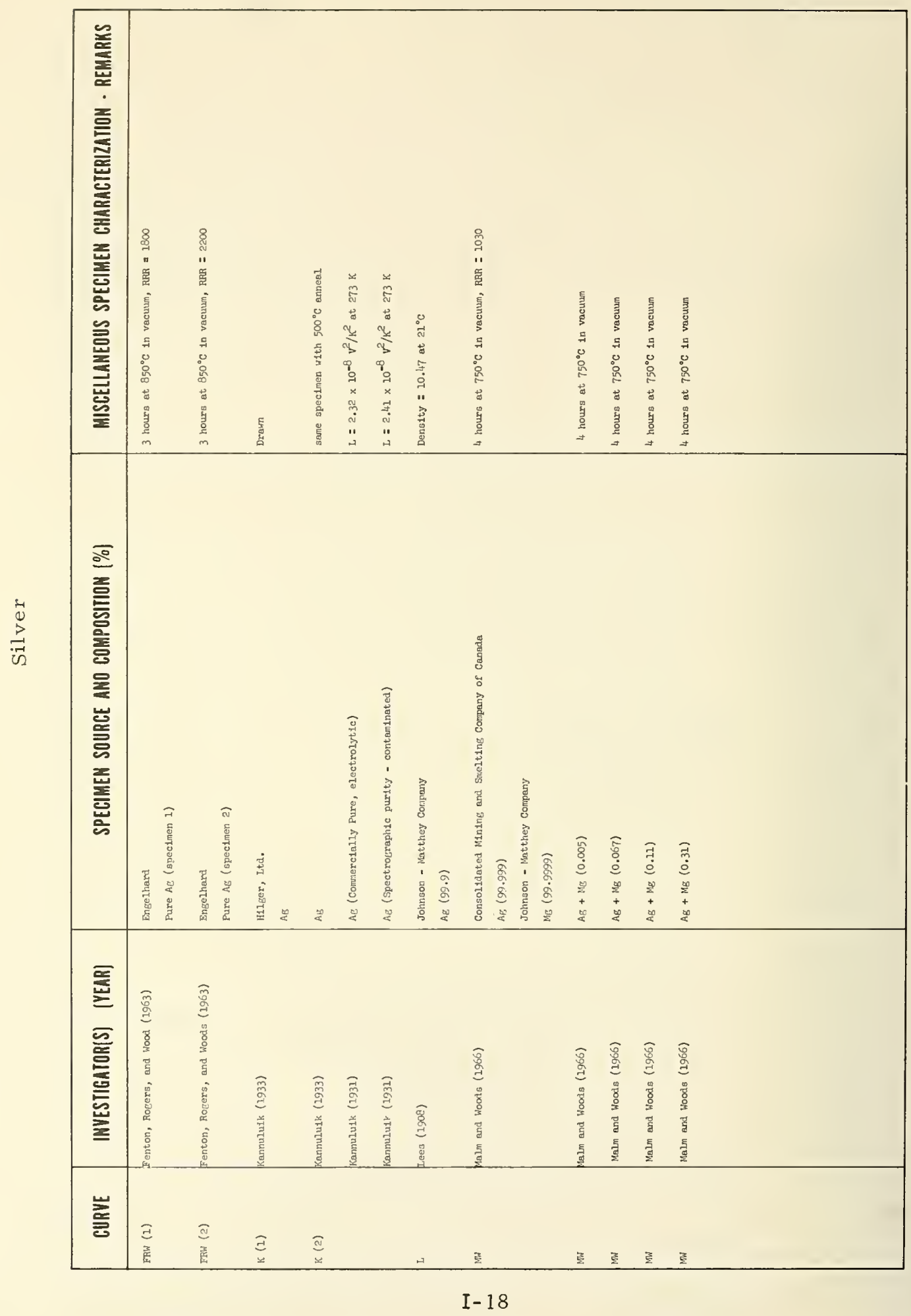




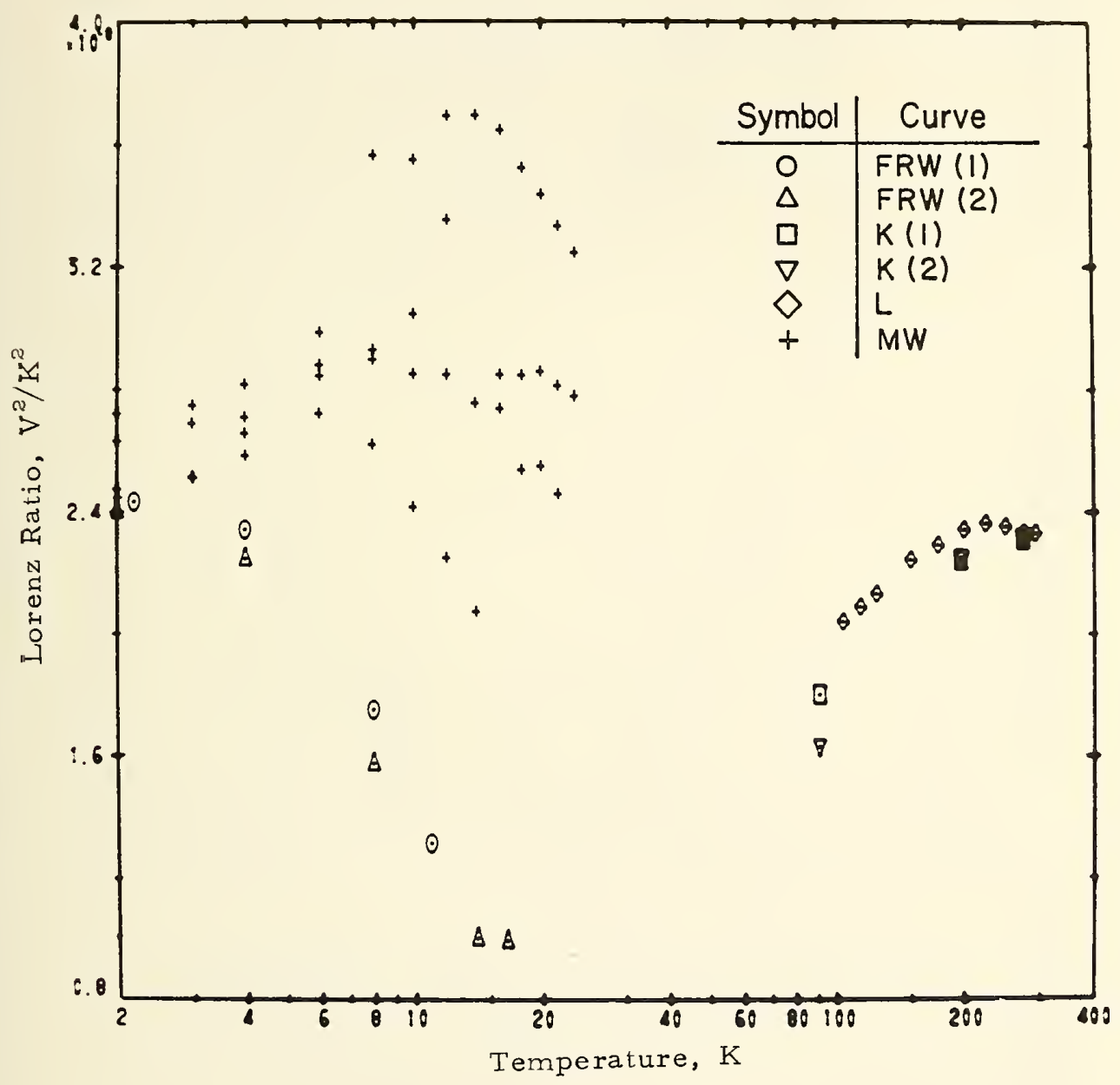

Figure 7. Lorenz ratio of silver 


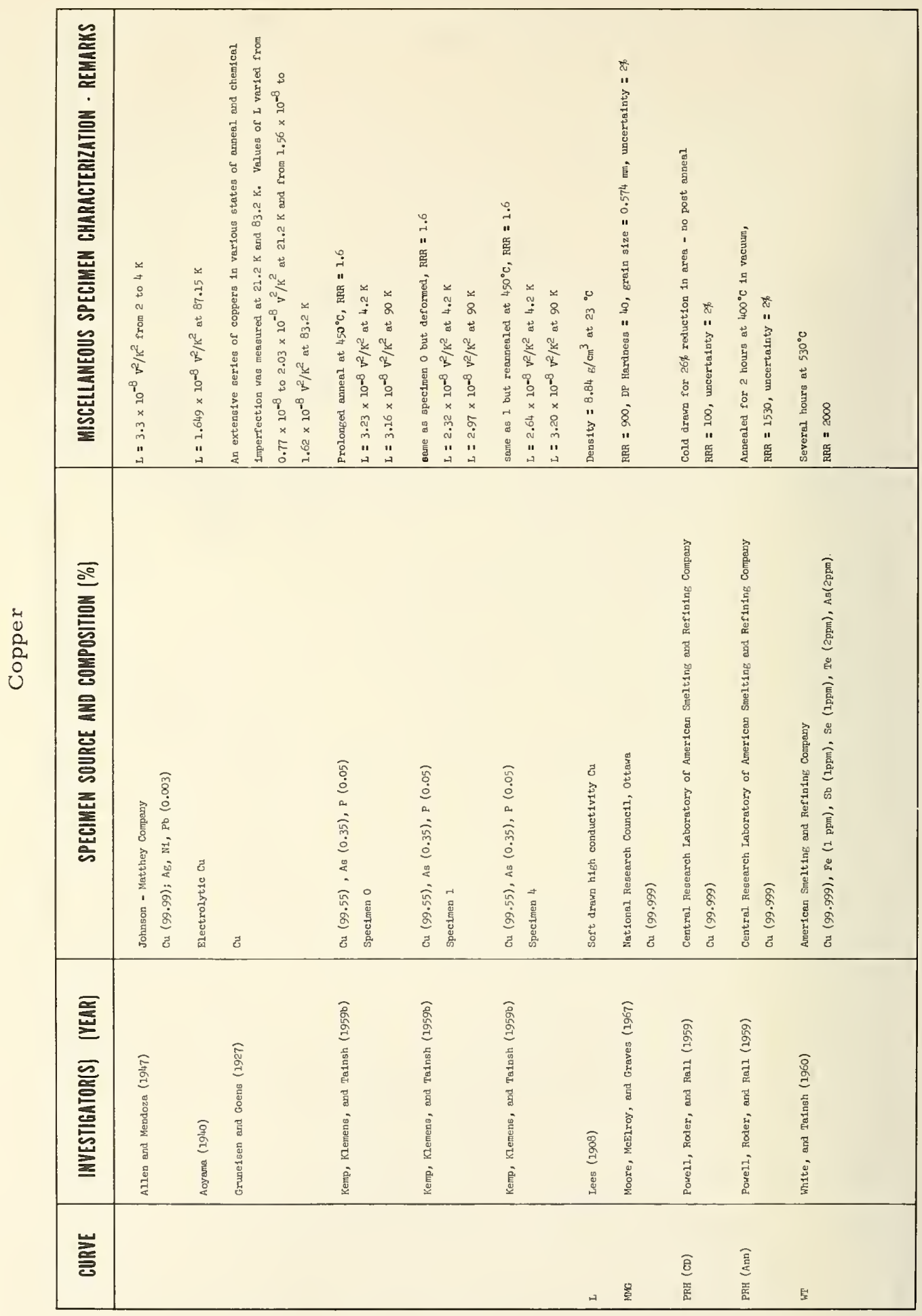




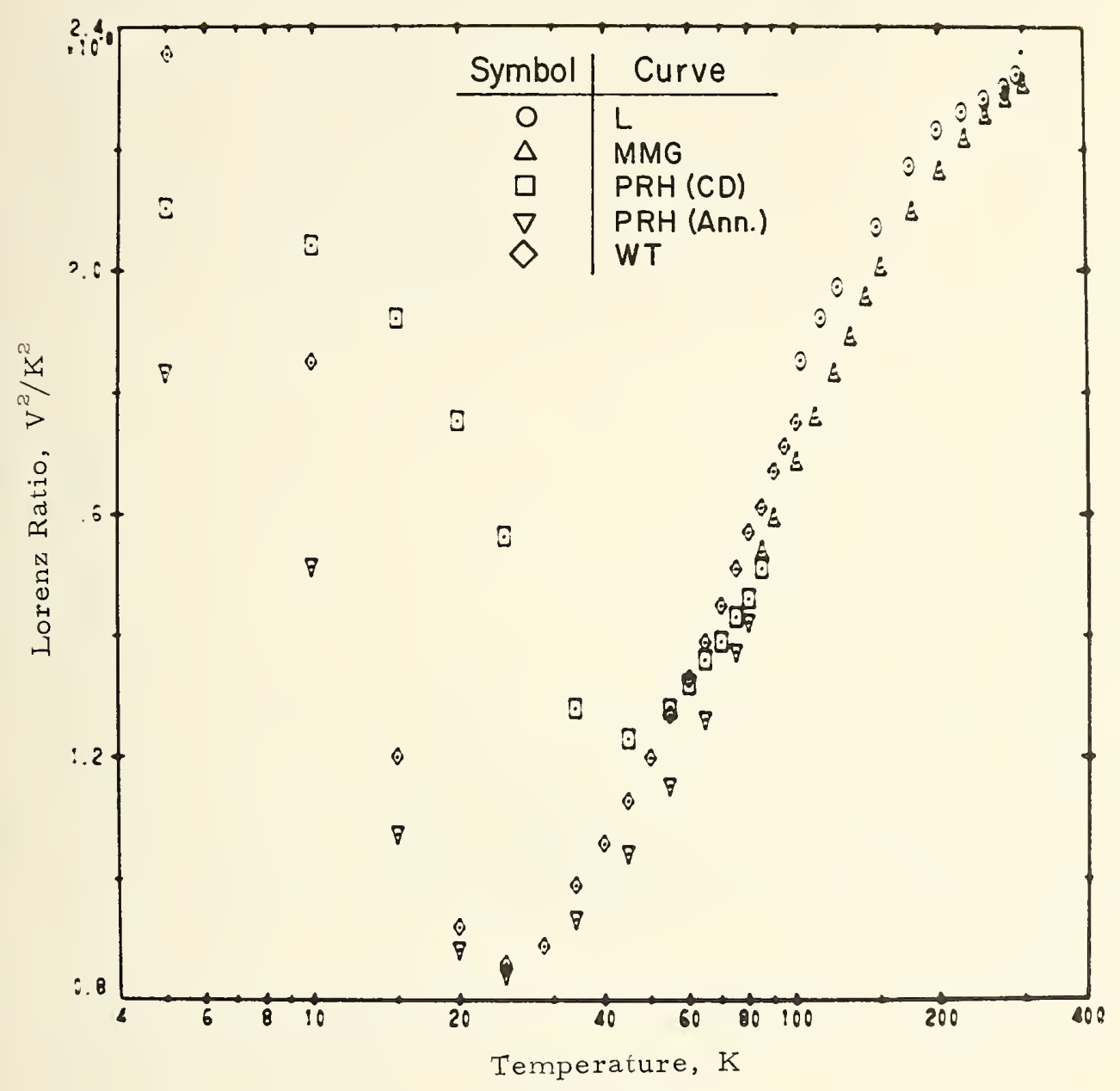

Figure 8. Lorenz ratio of copper 


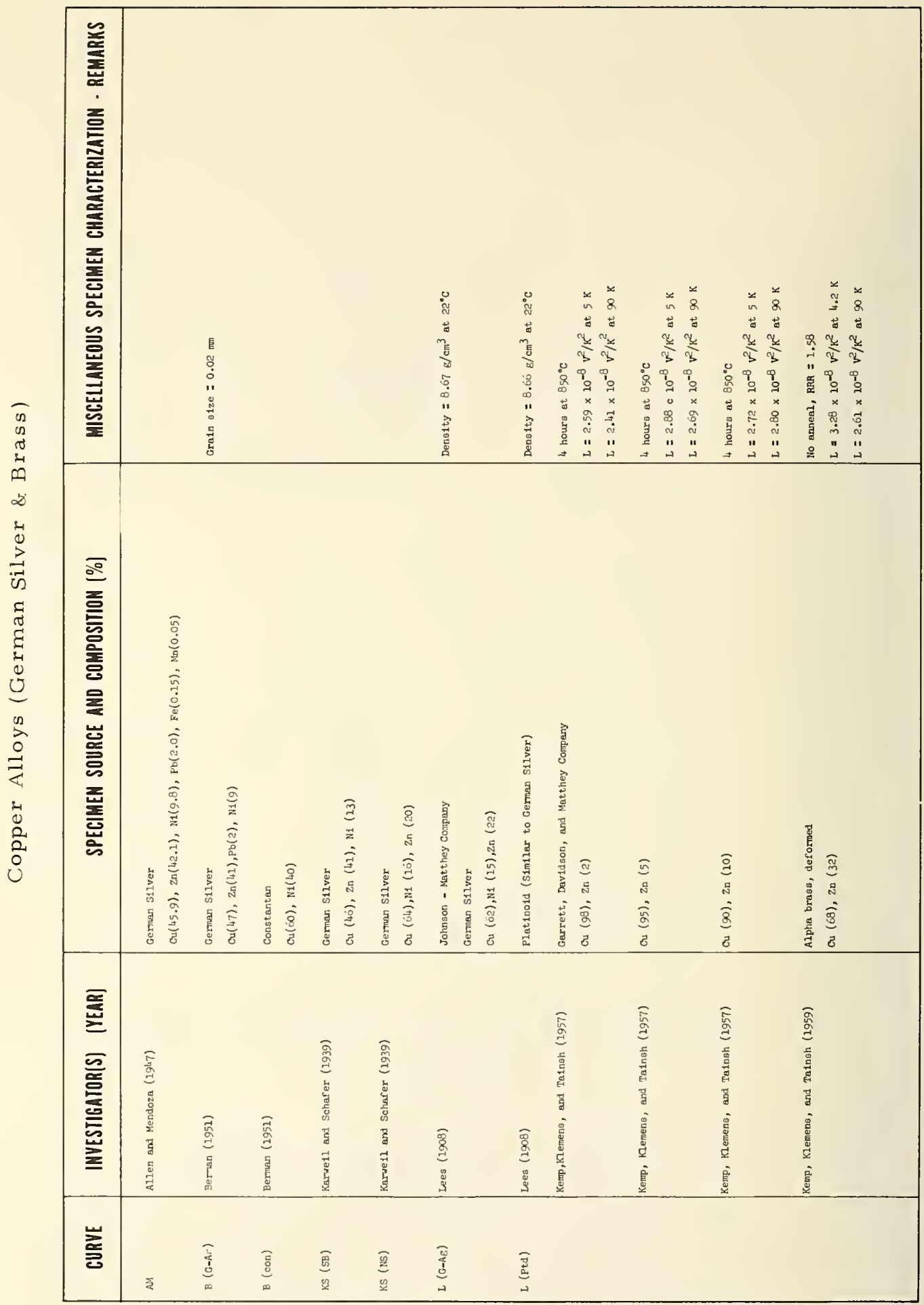




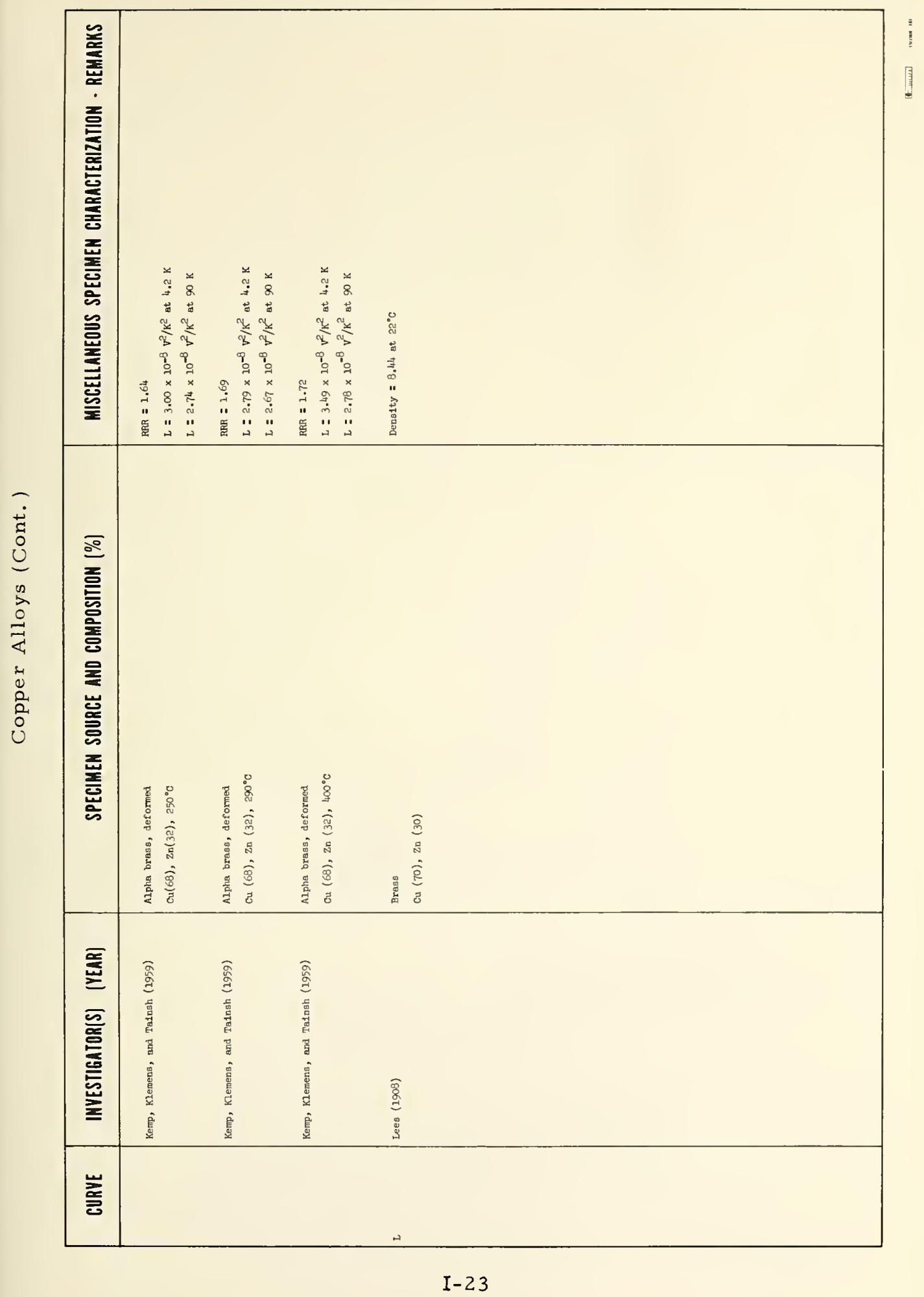




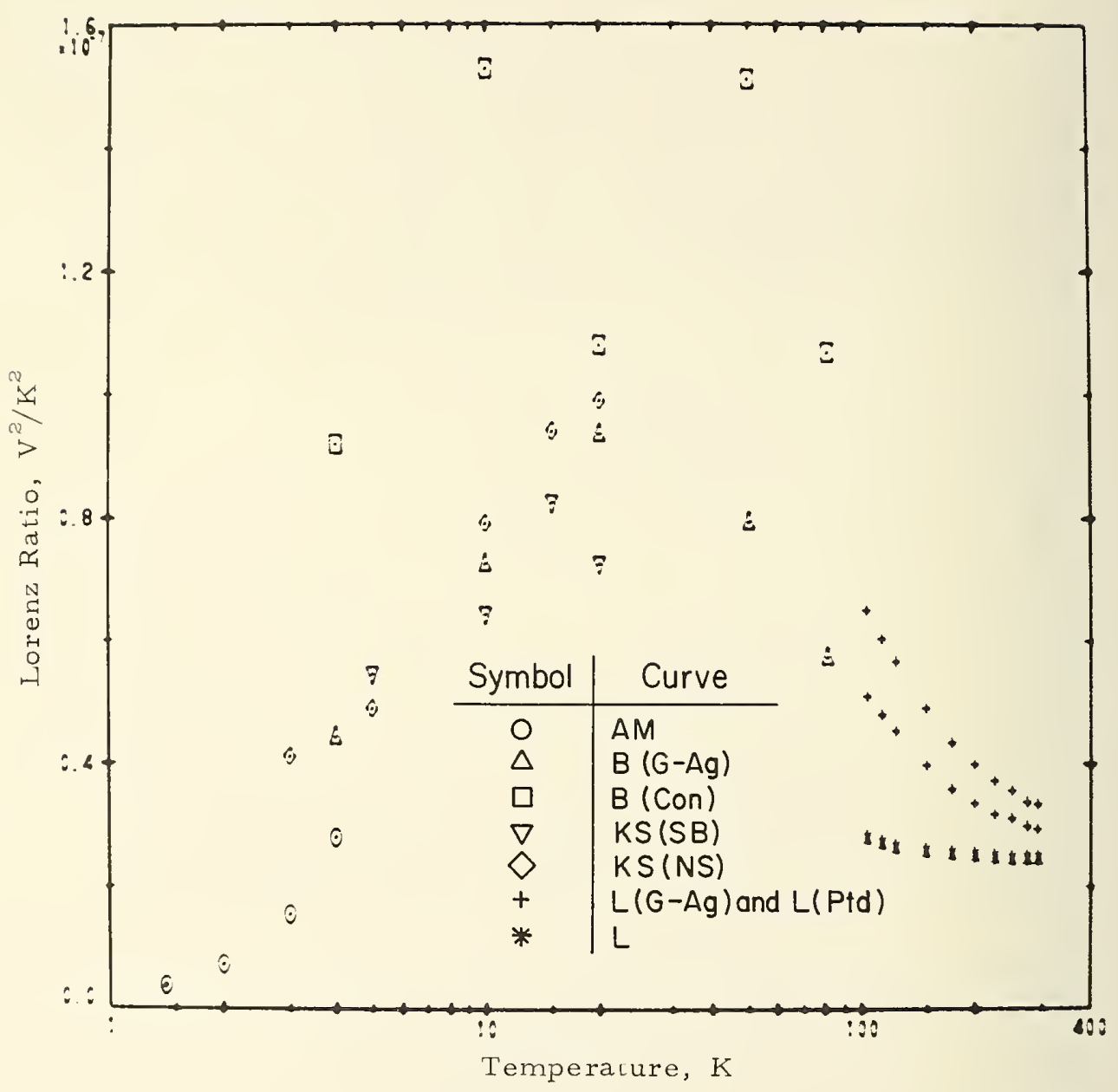

Figure 9. Lorenz ratio of copper alloys (German silver and brass) 


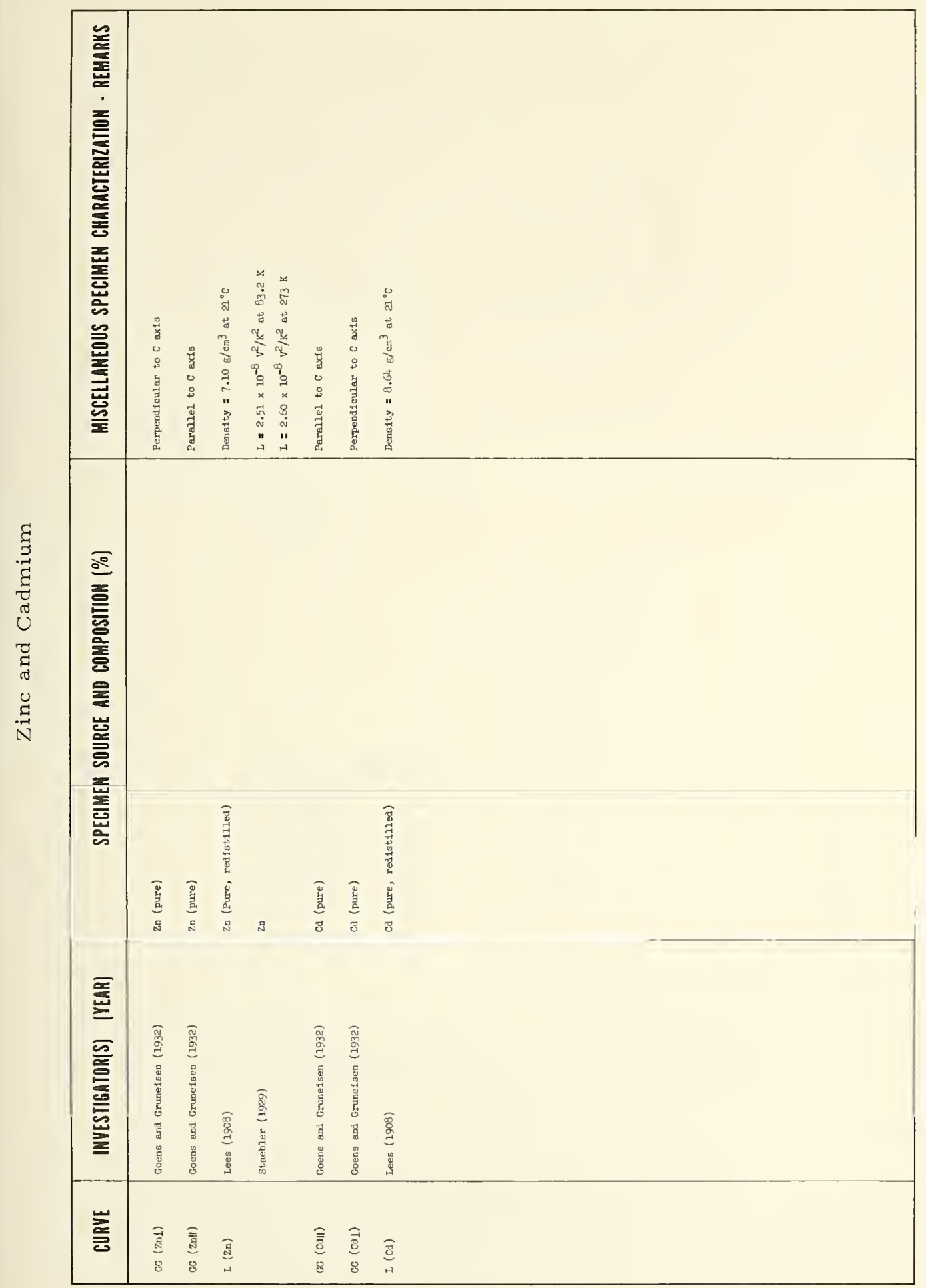




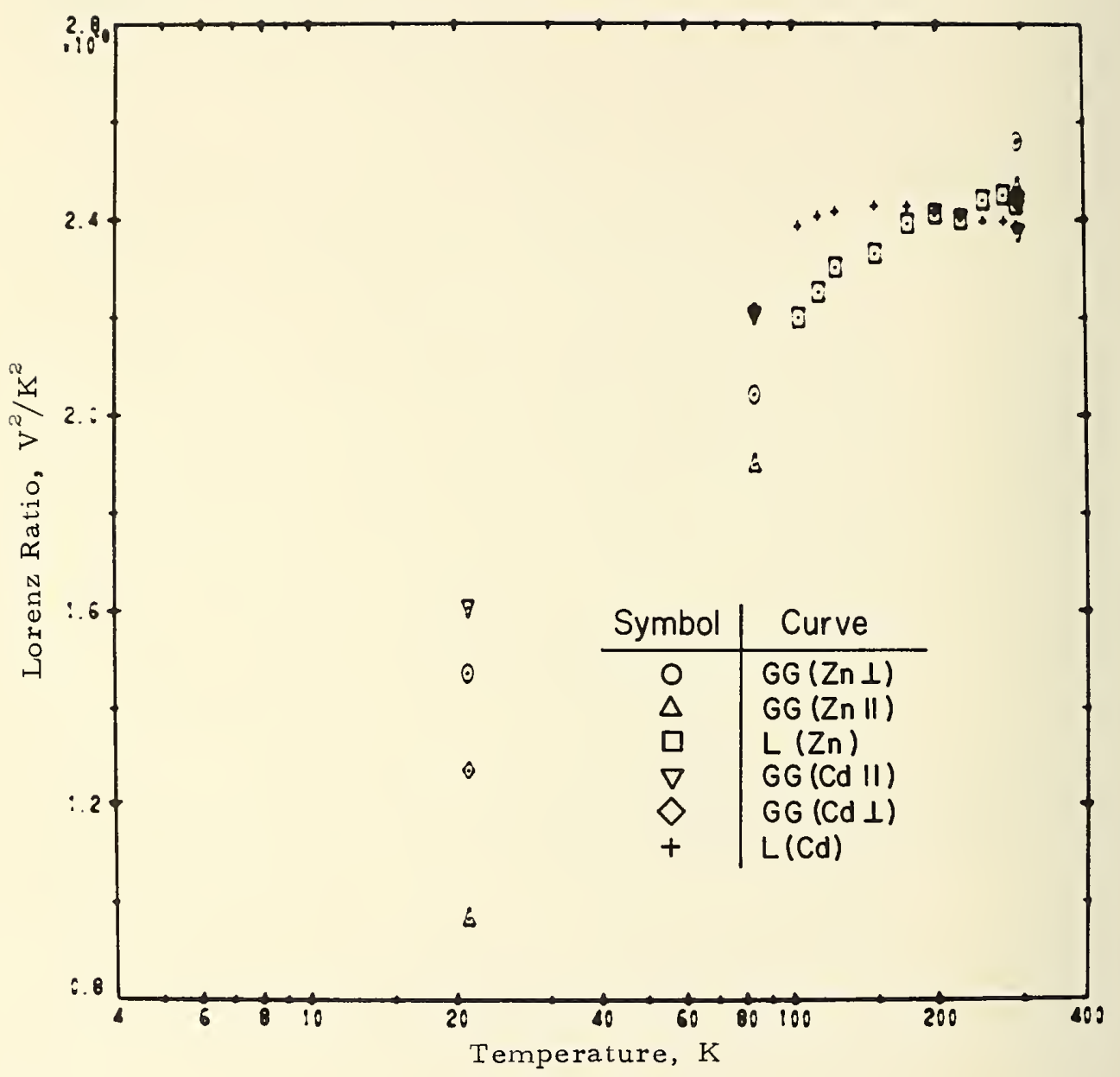

Figure 10. Lorenz ratio of zinc and cadmium 


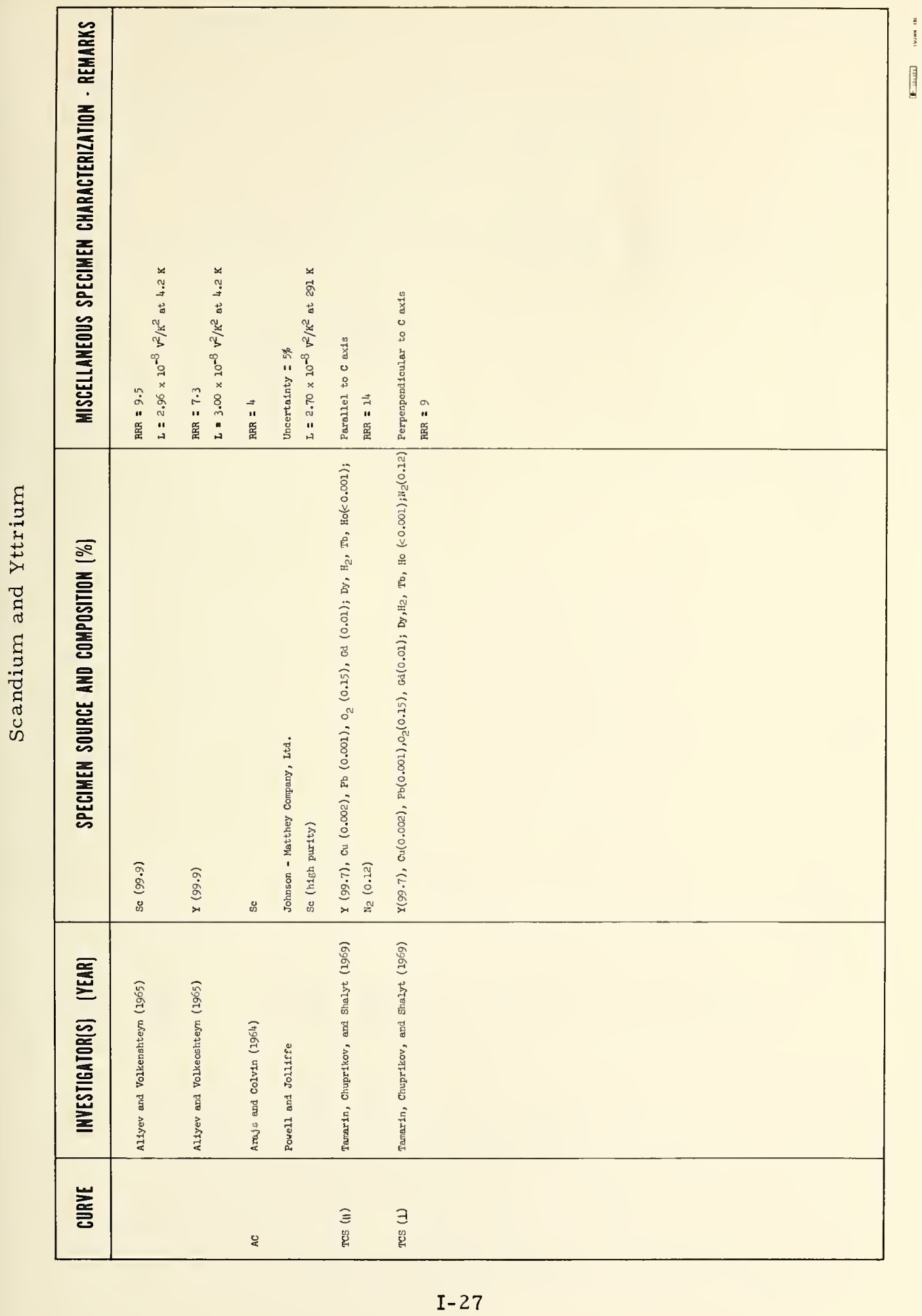




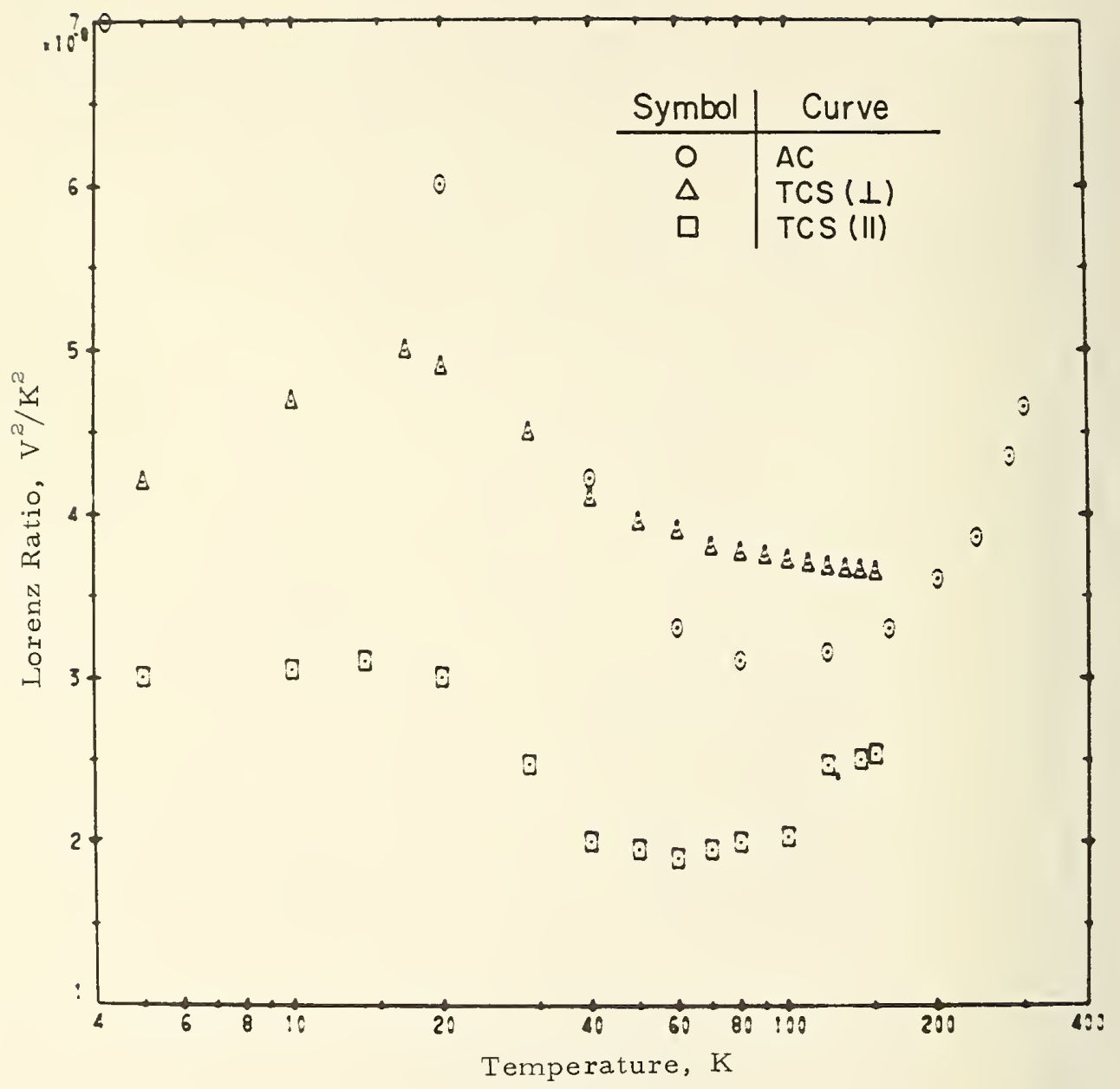

Figure 11. Lorenz ratio of scandium and yttrium 


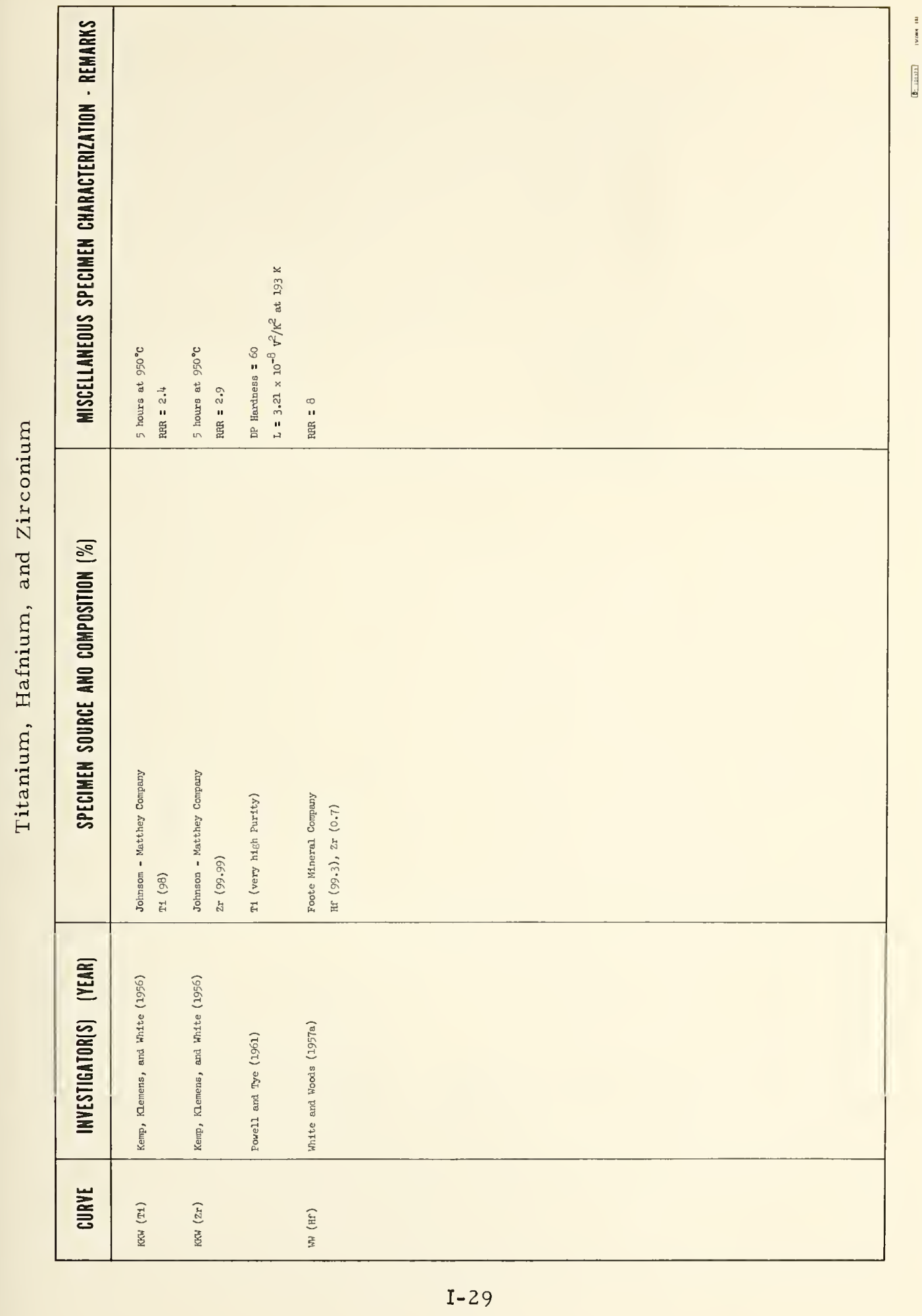




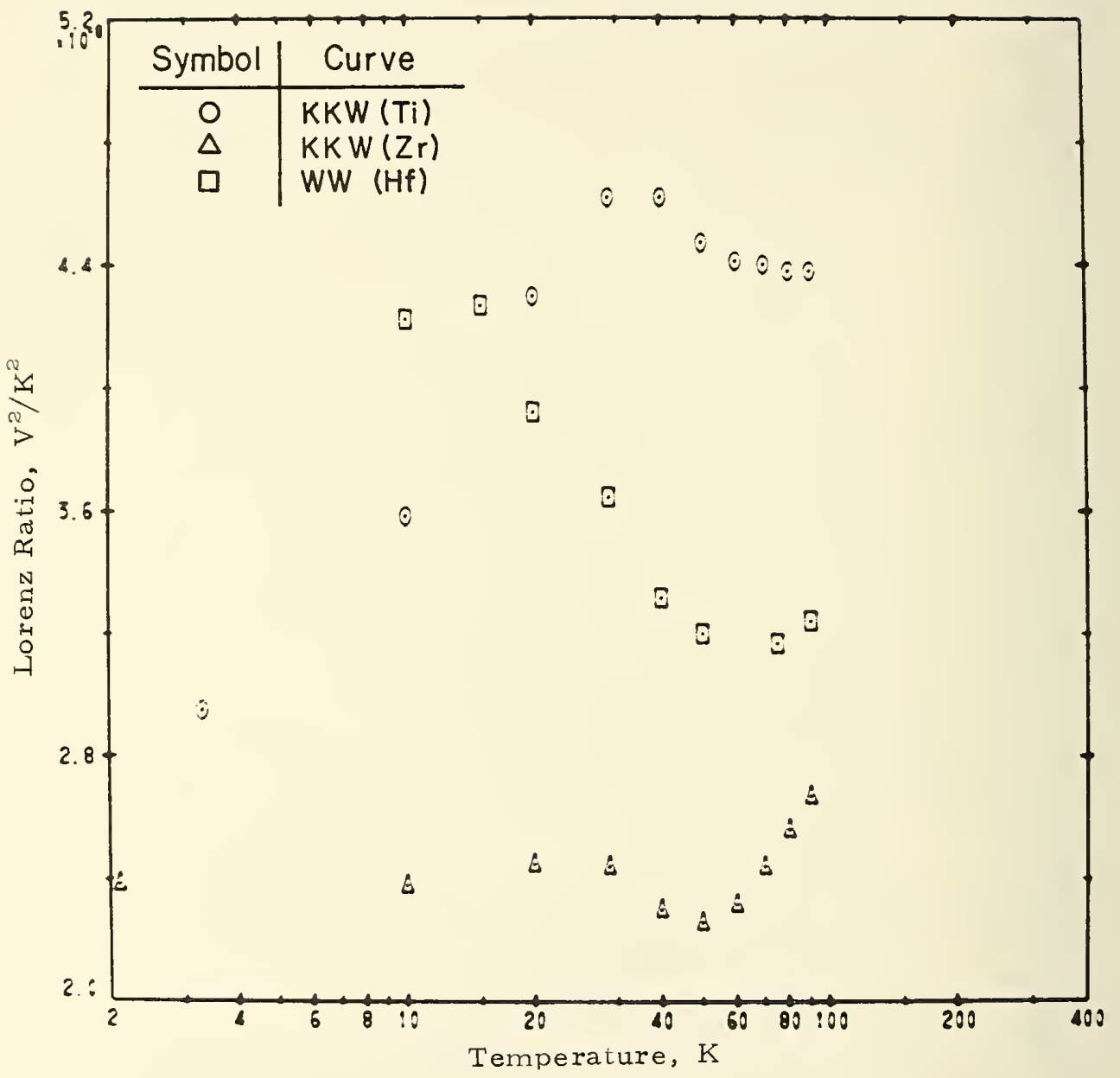

Figure 12. Lorenz ratio of titanium, hafnium, and zirconium 


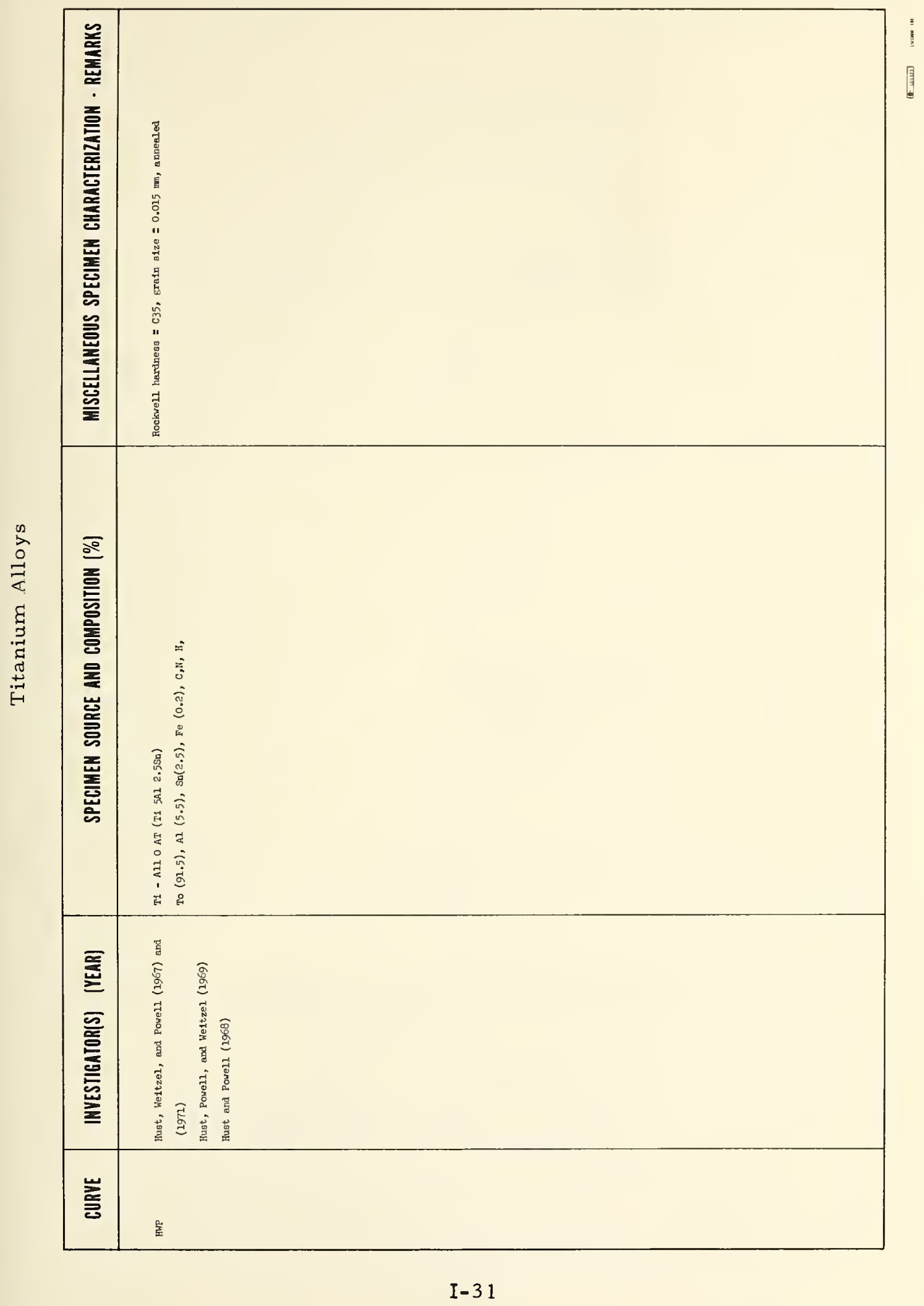




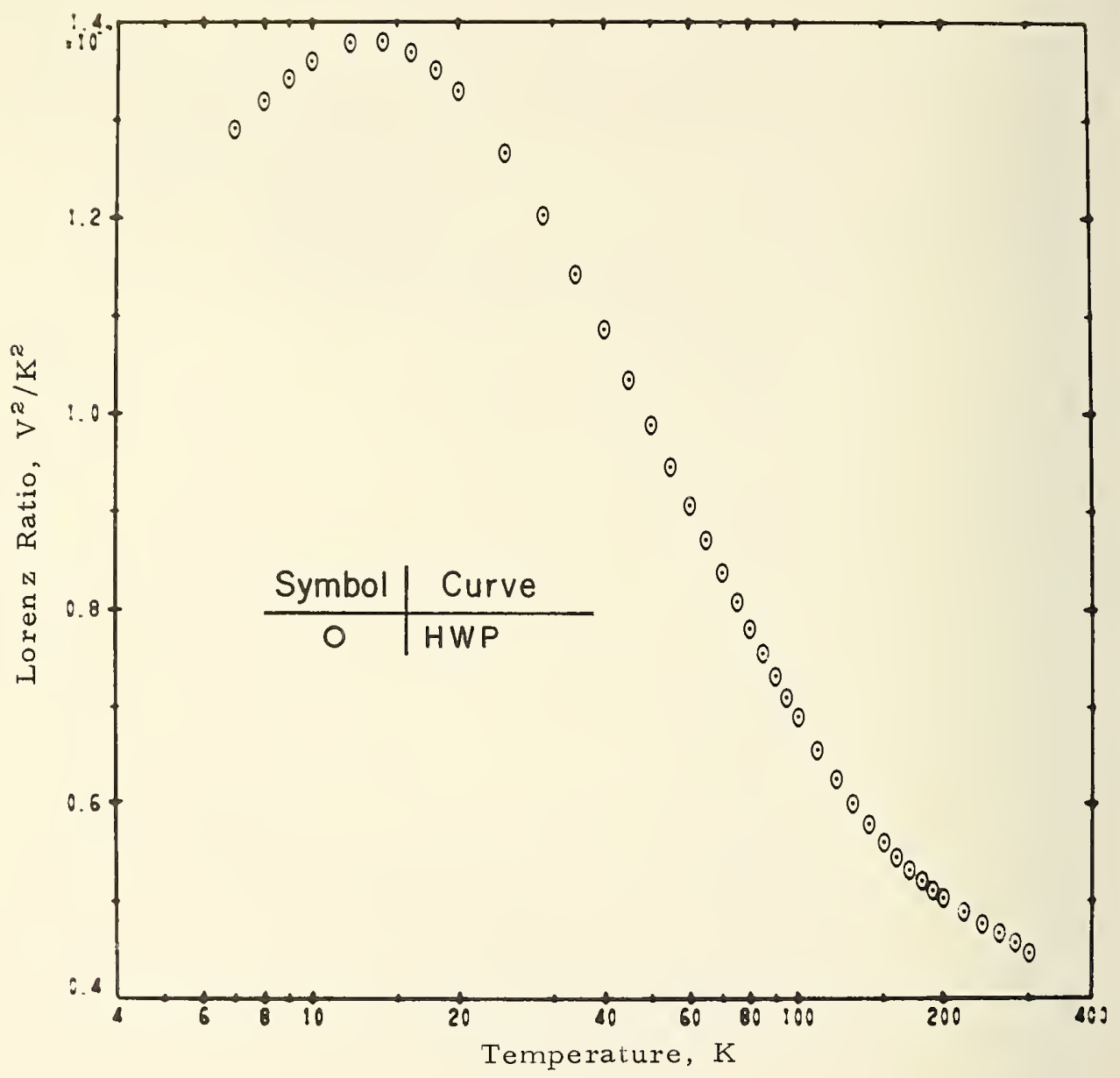

Figure 13. Lorenz ratio of titanium alloys 


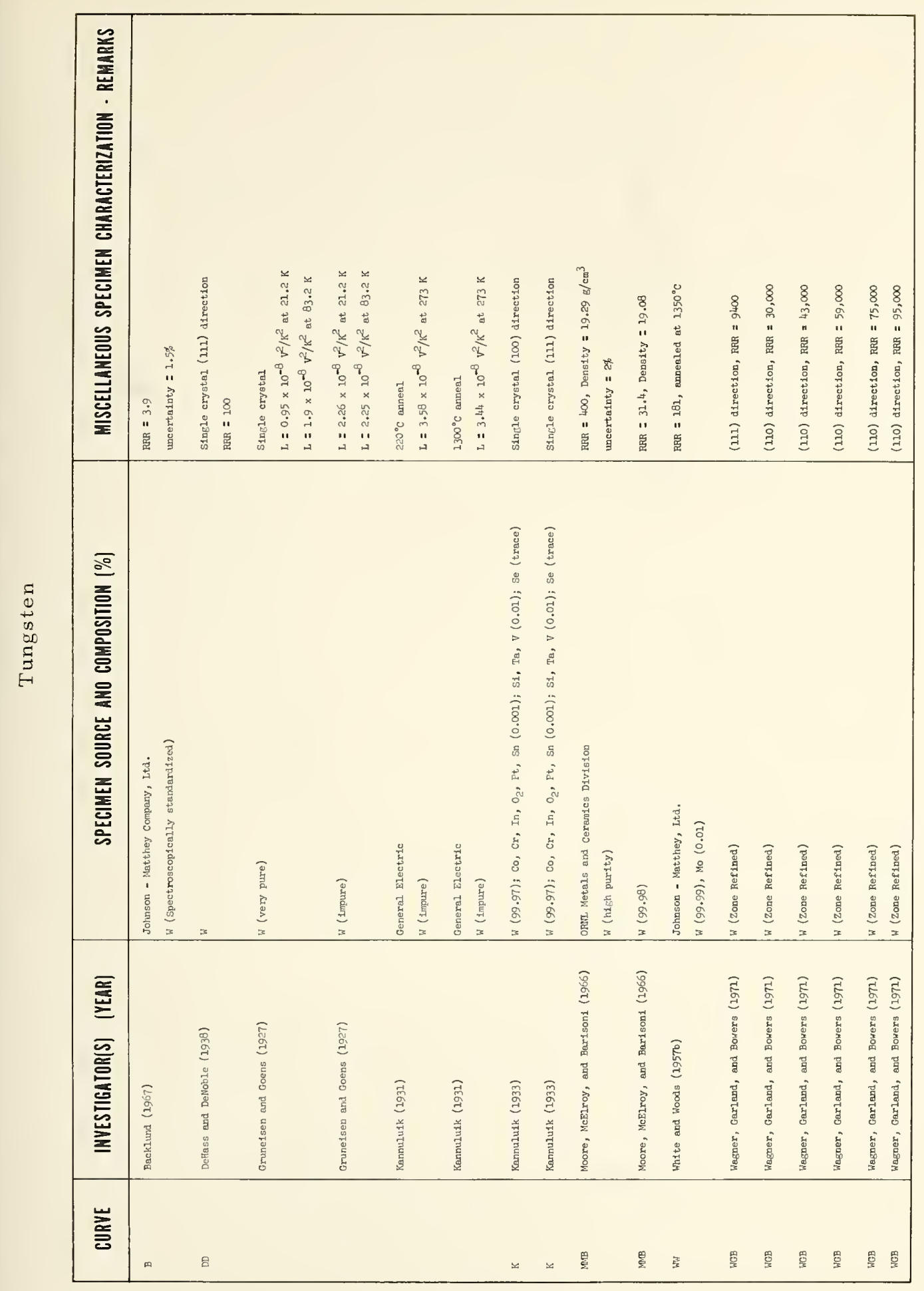




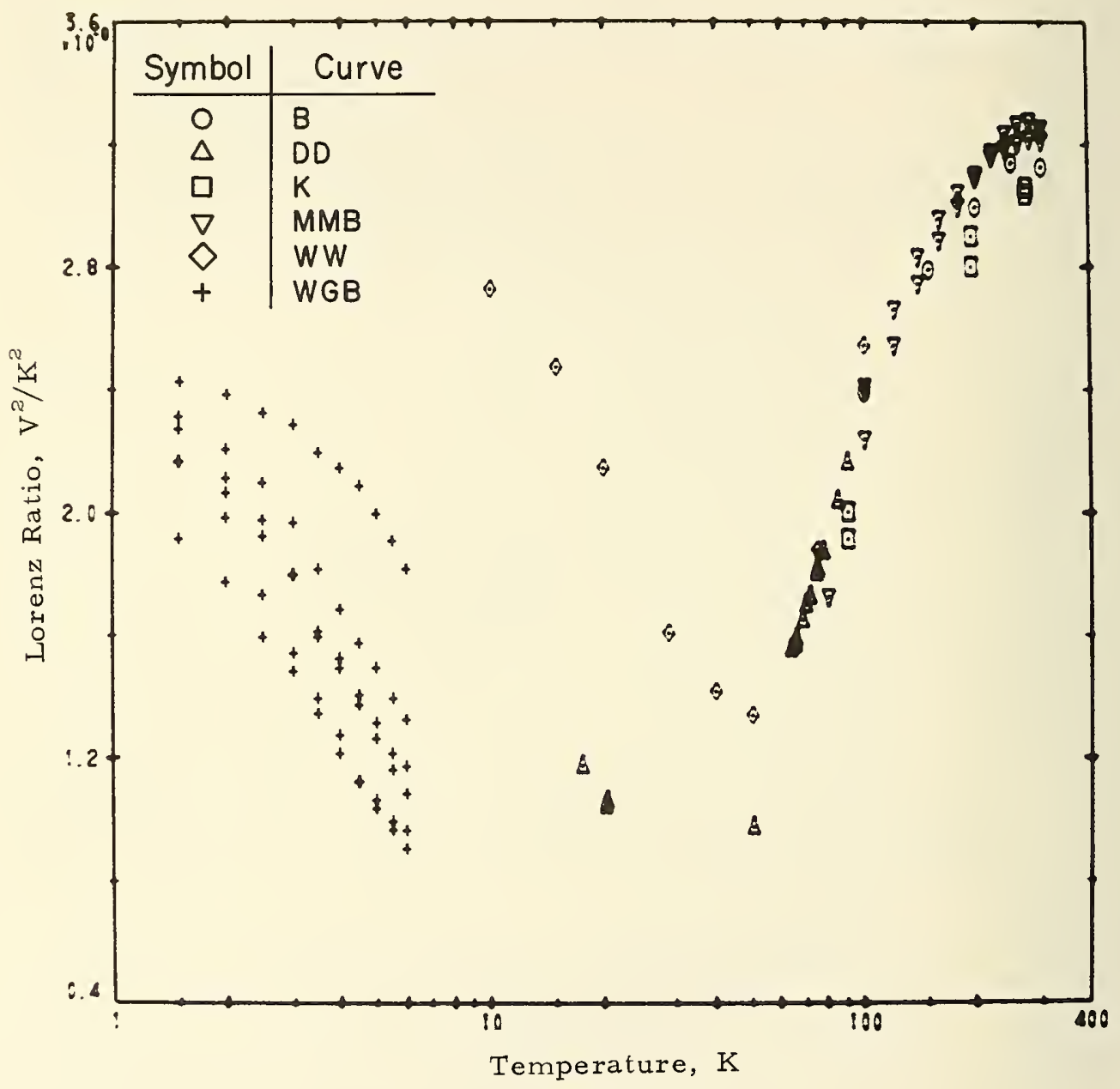

Figure 14. Lorenz ratio of tungsten 


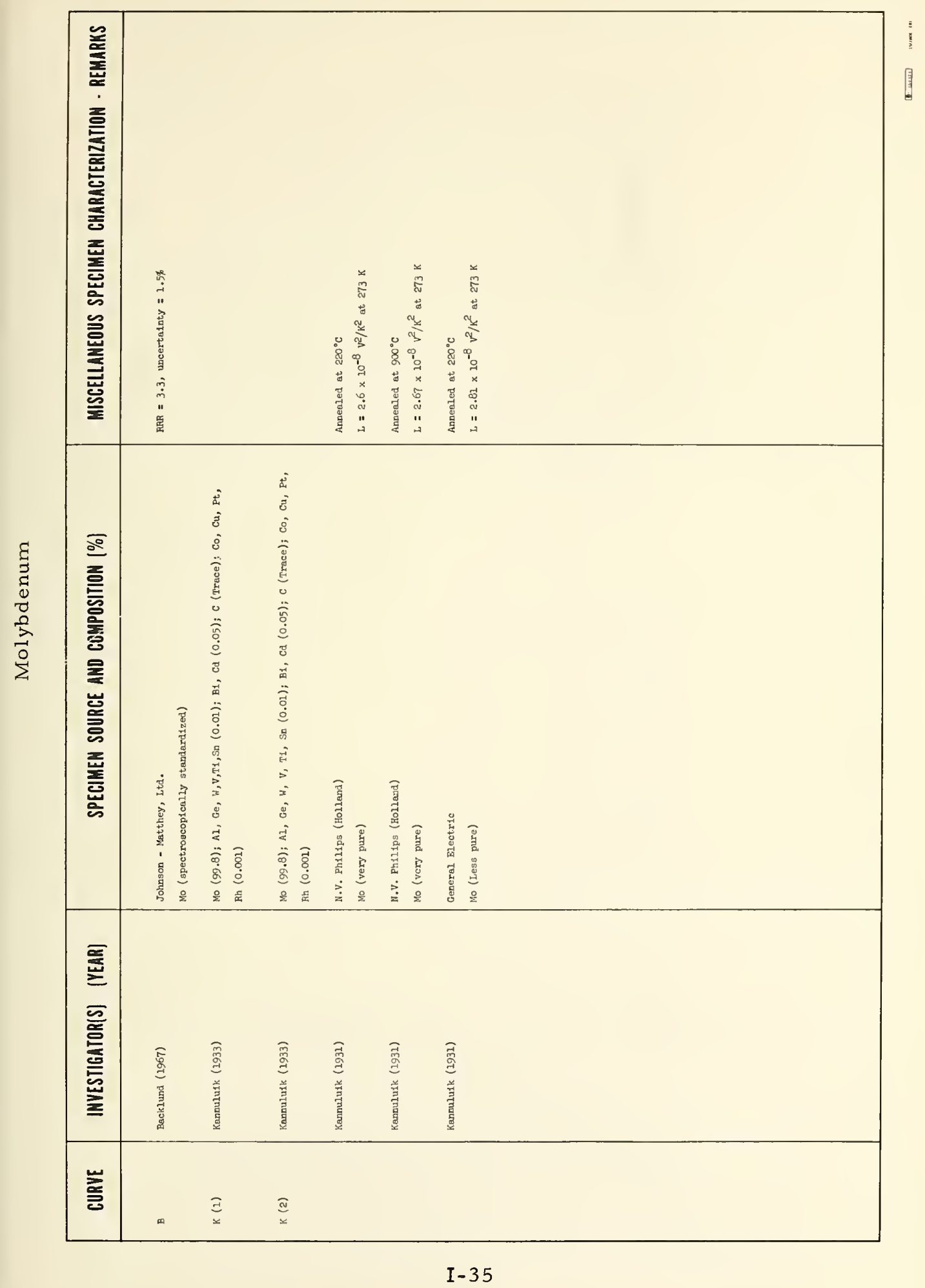




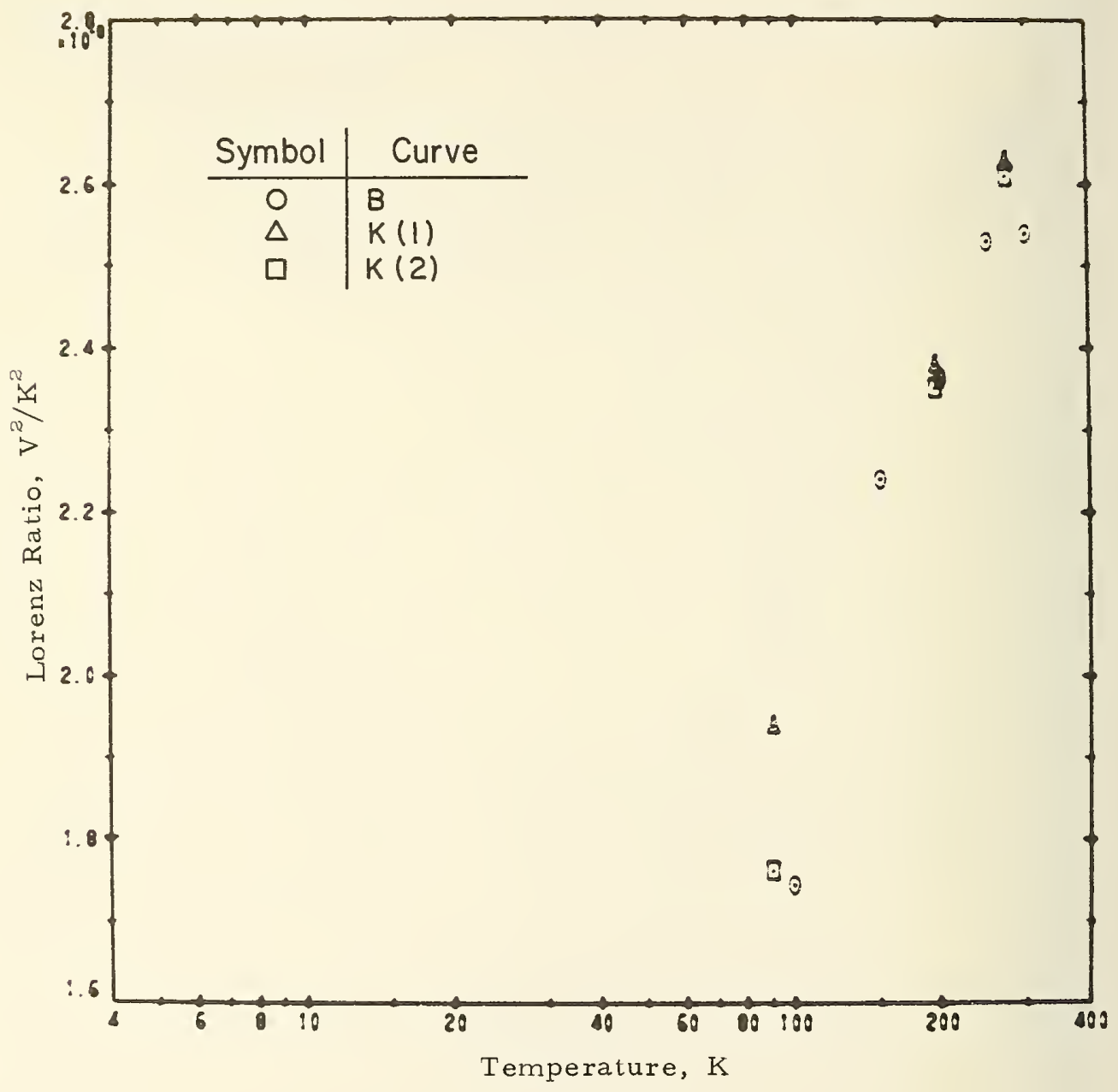

Figure 15. Lorenz ratio of molybdenum 


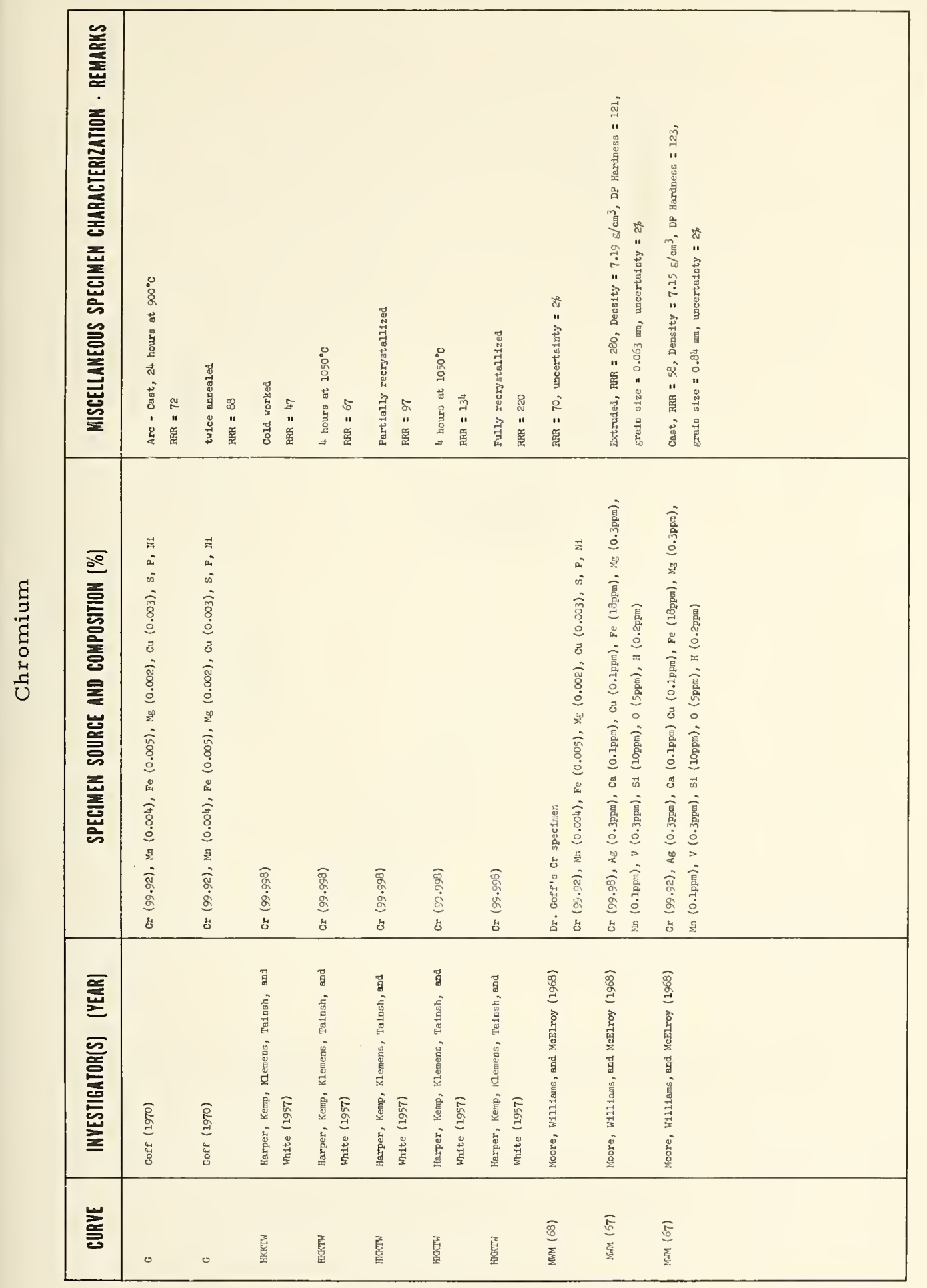




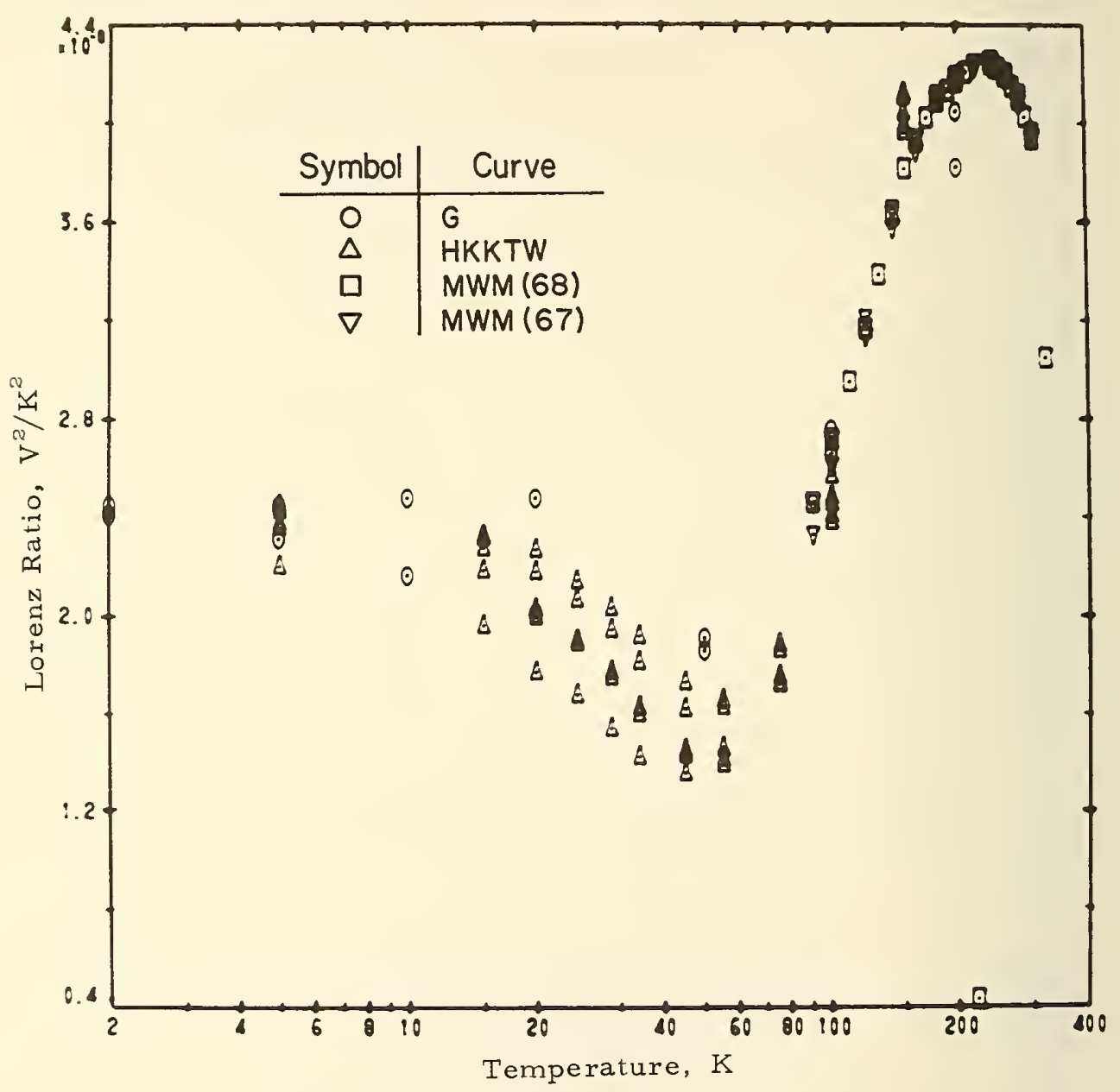

Figure 16. Lorenz ratio of chromium 


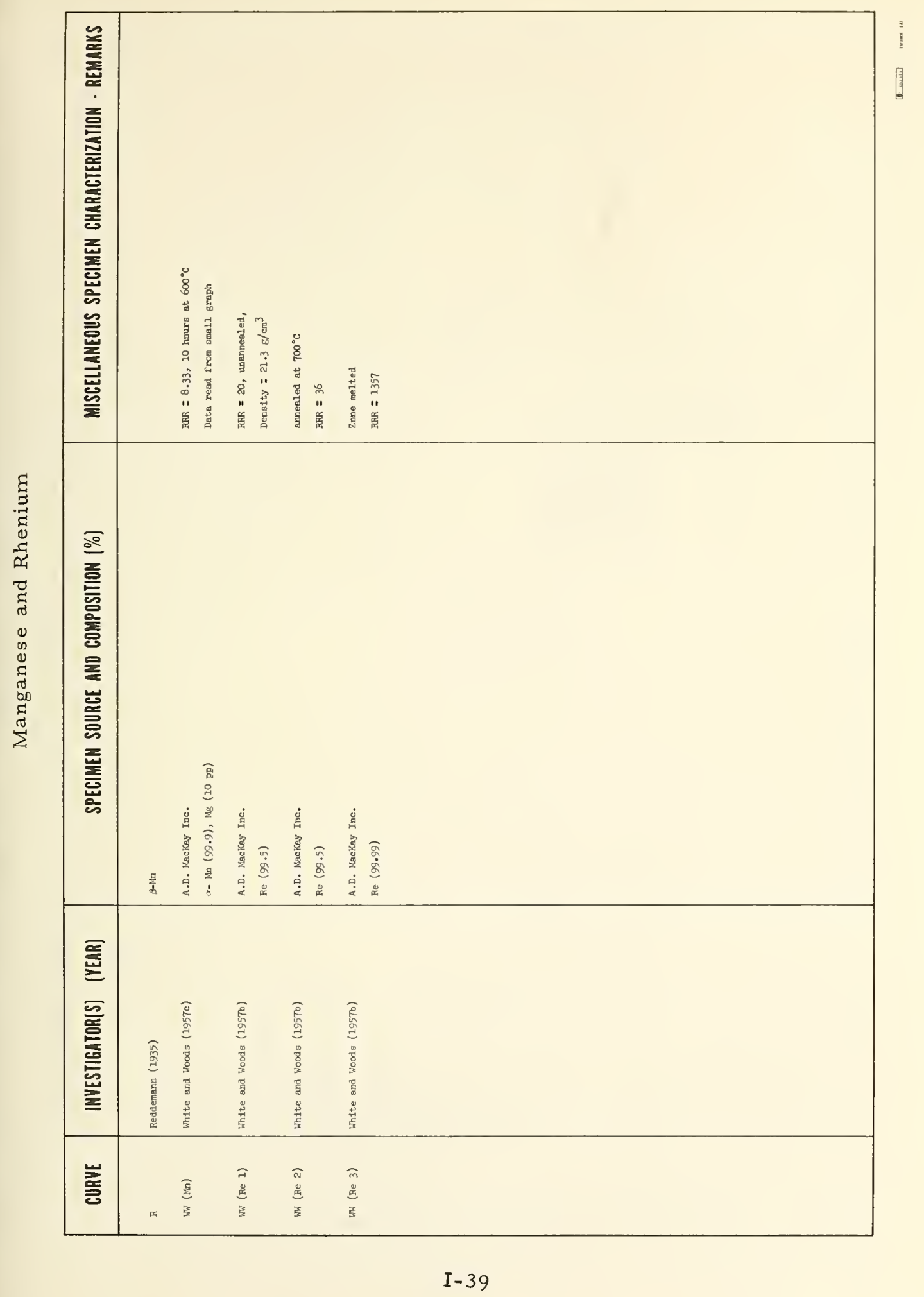




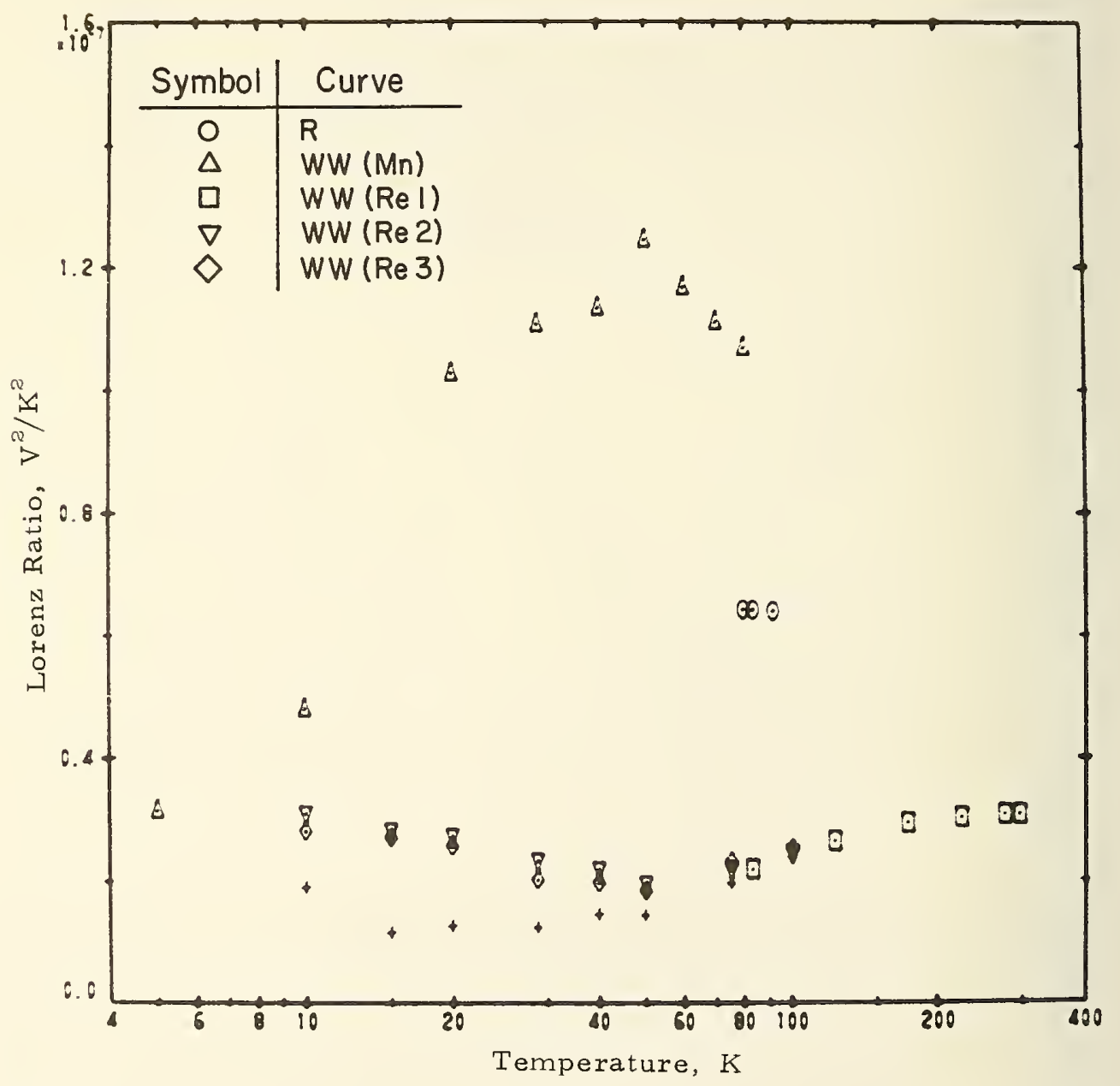

Figure 17. Lorenz ratio of manganese and rhenium 


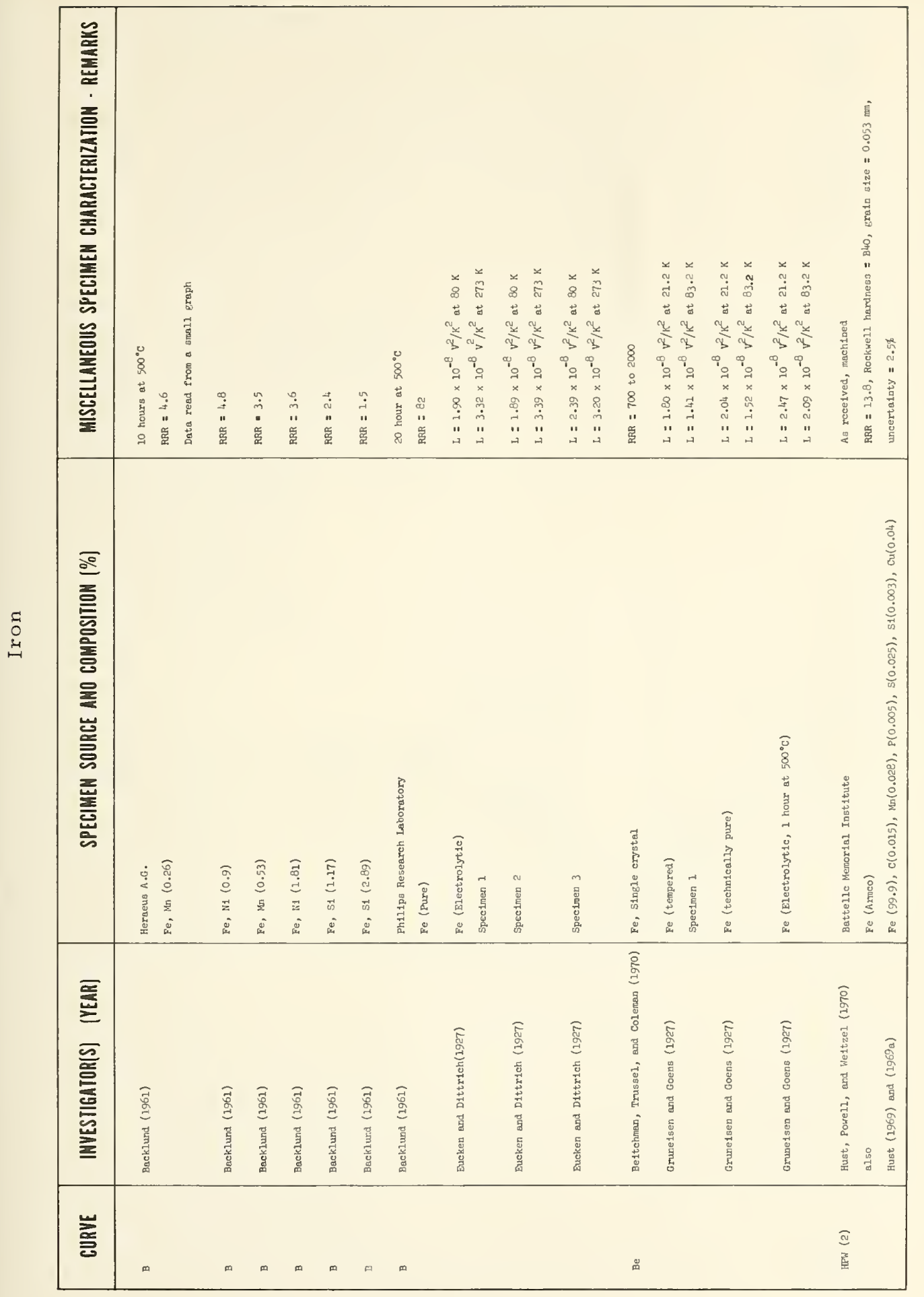




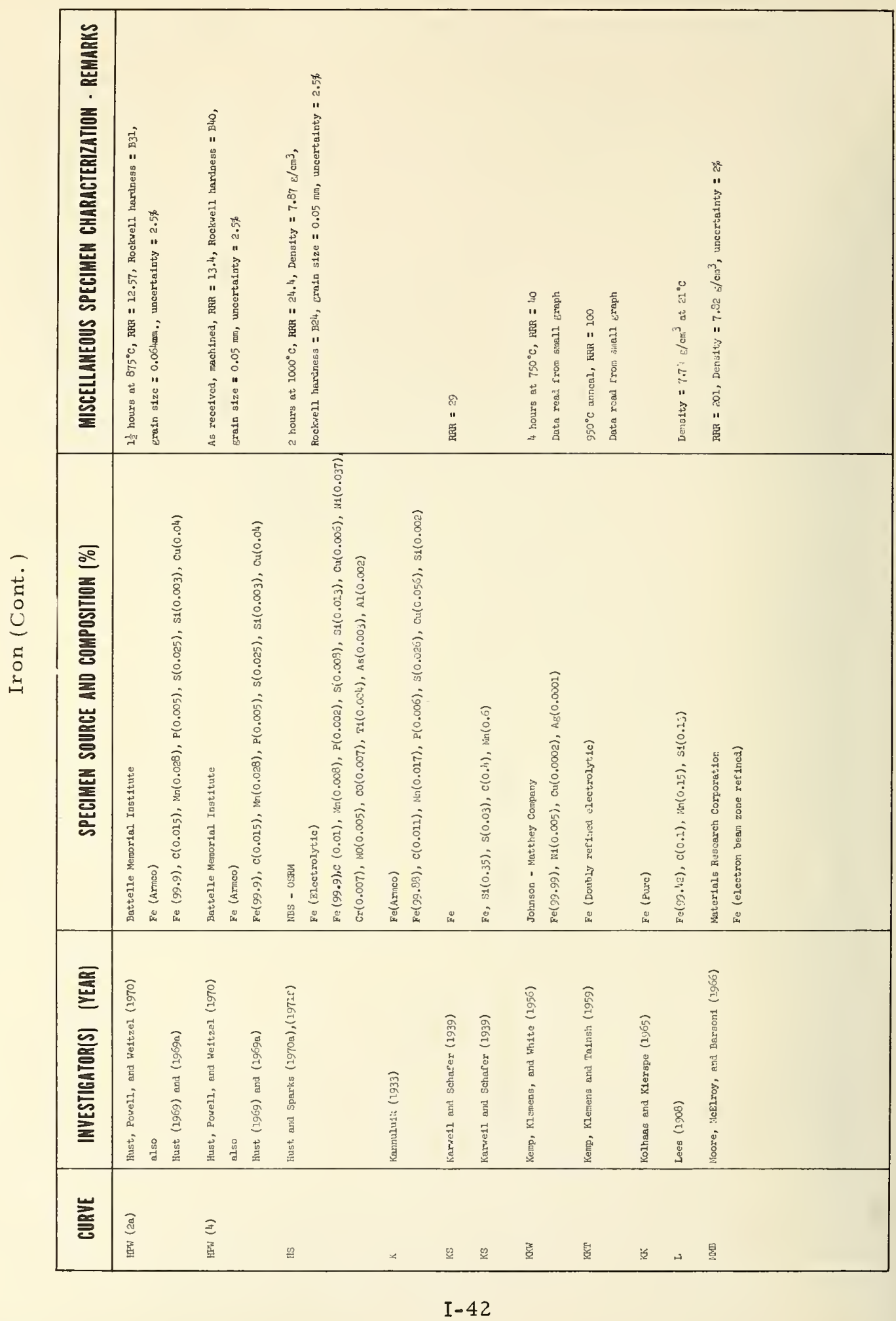




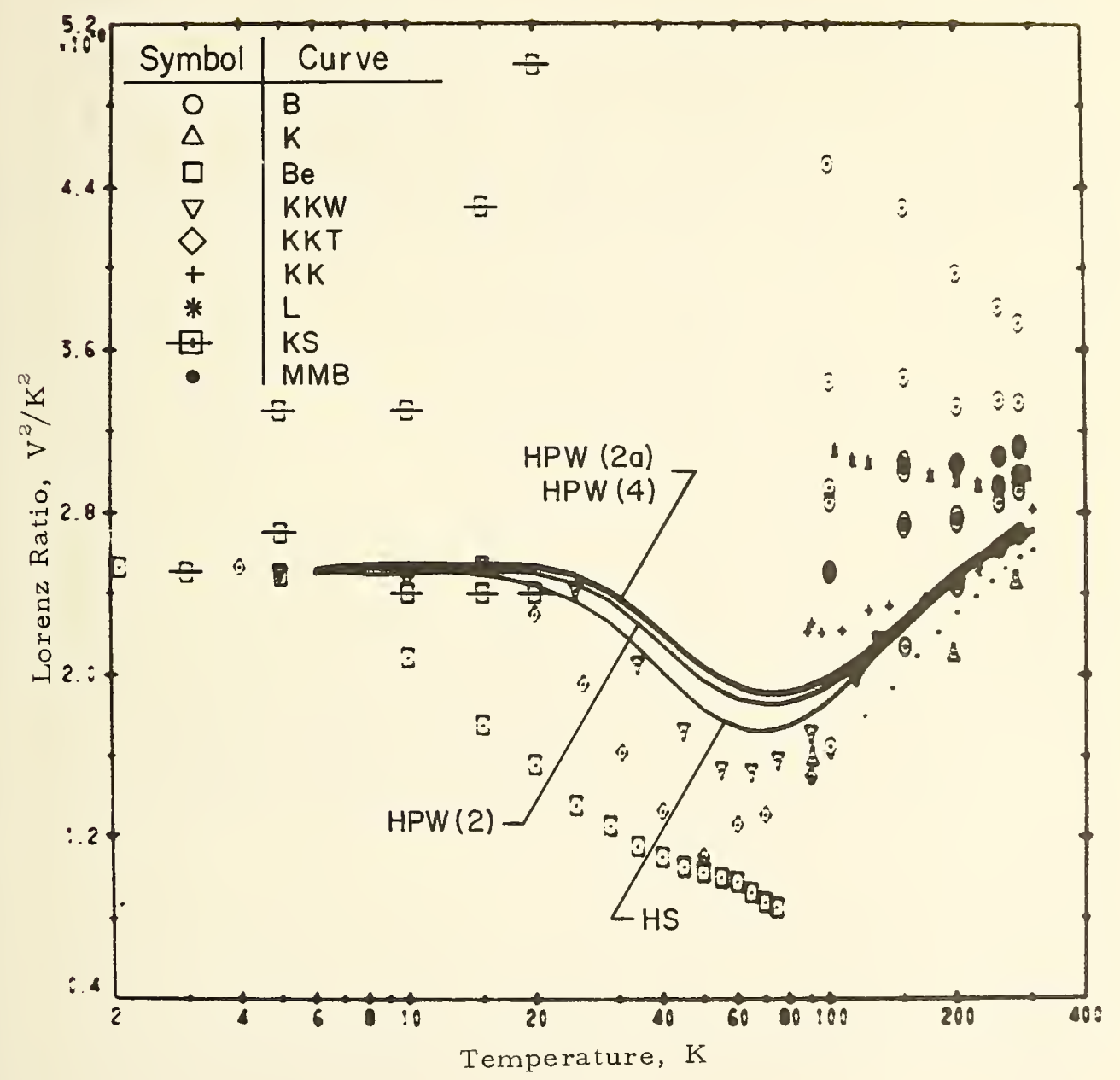

Figure 18. Lorenz ratio of iron 


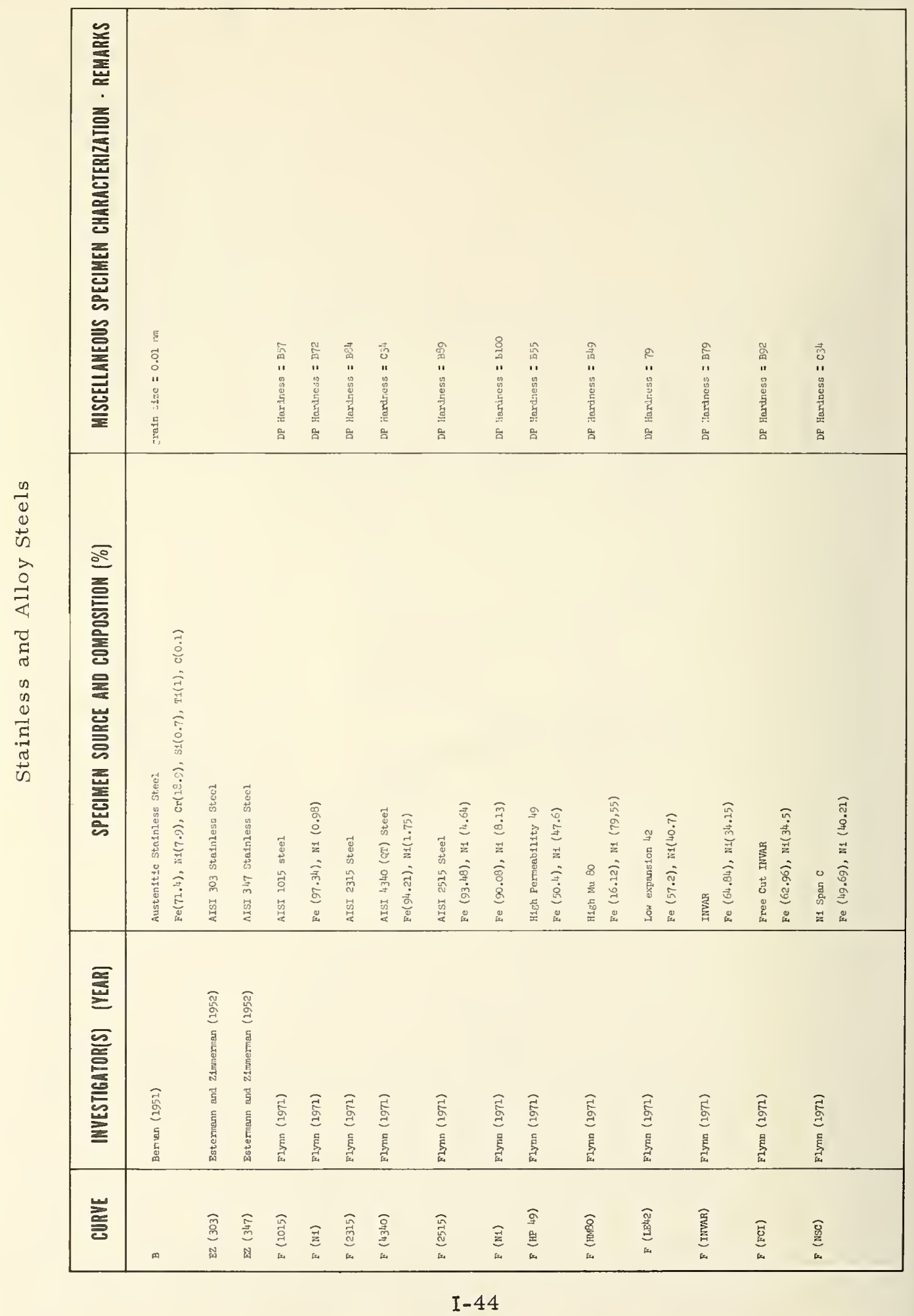




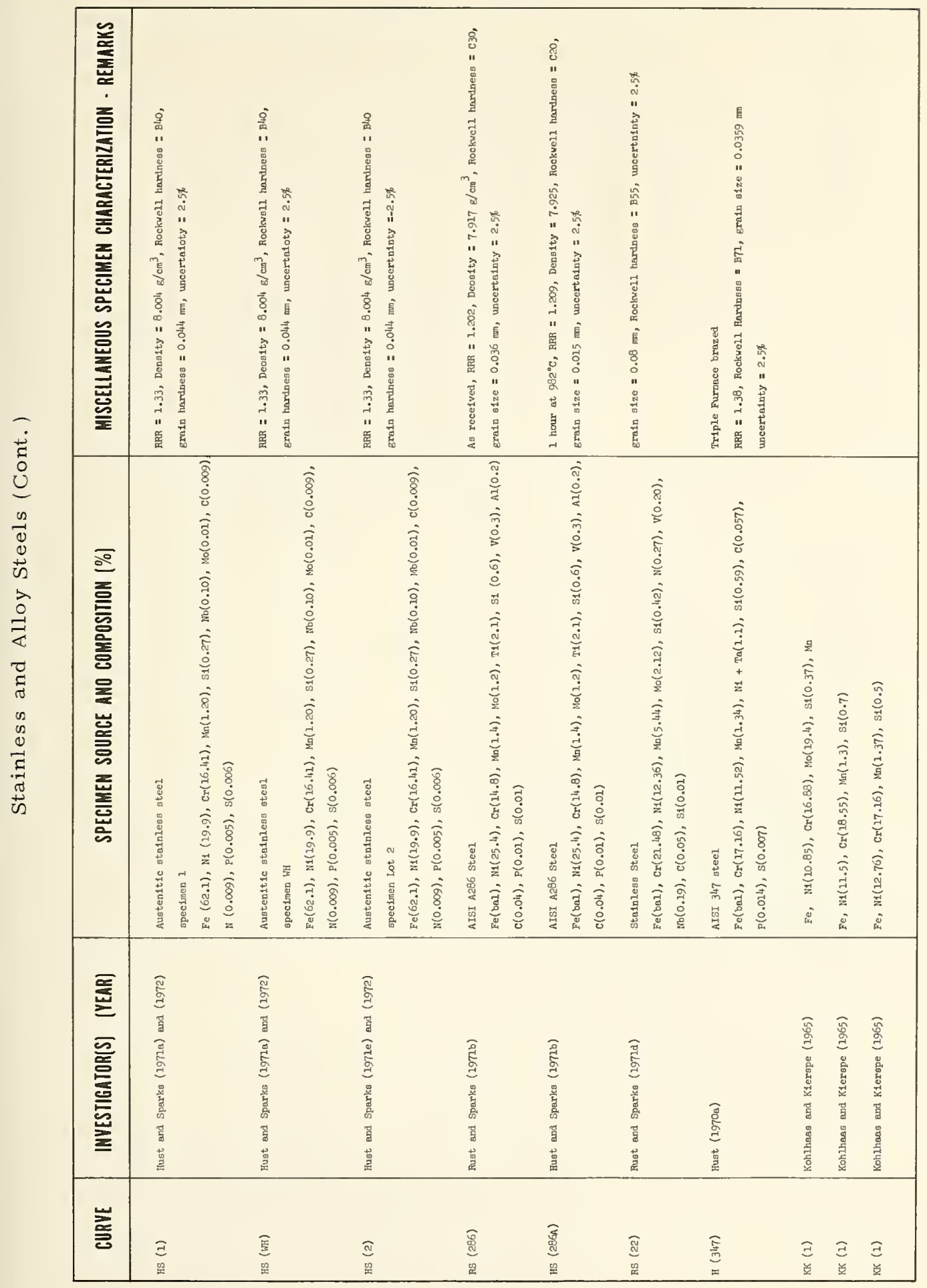




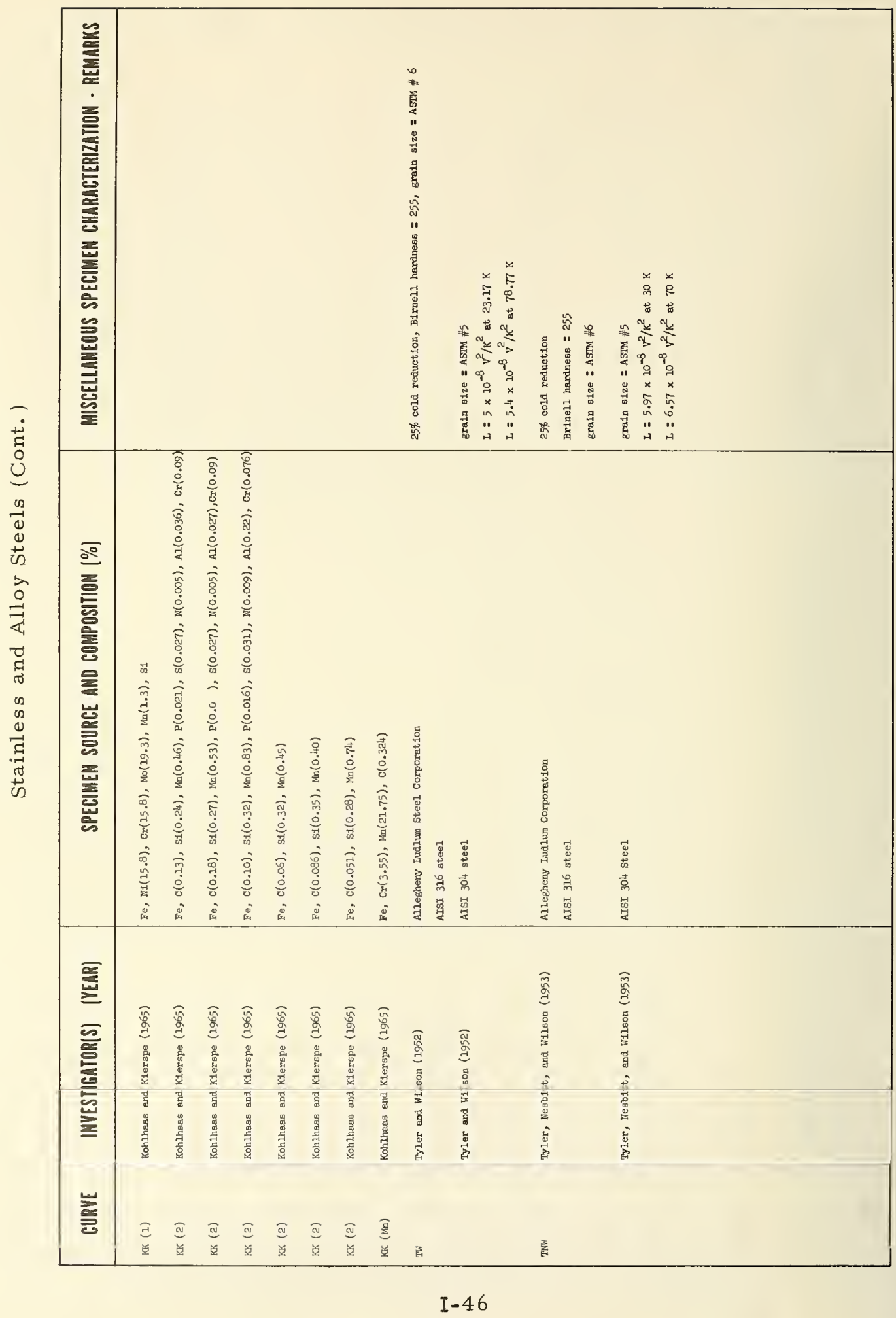




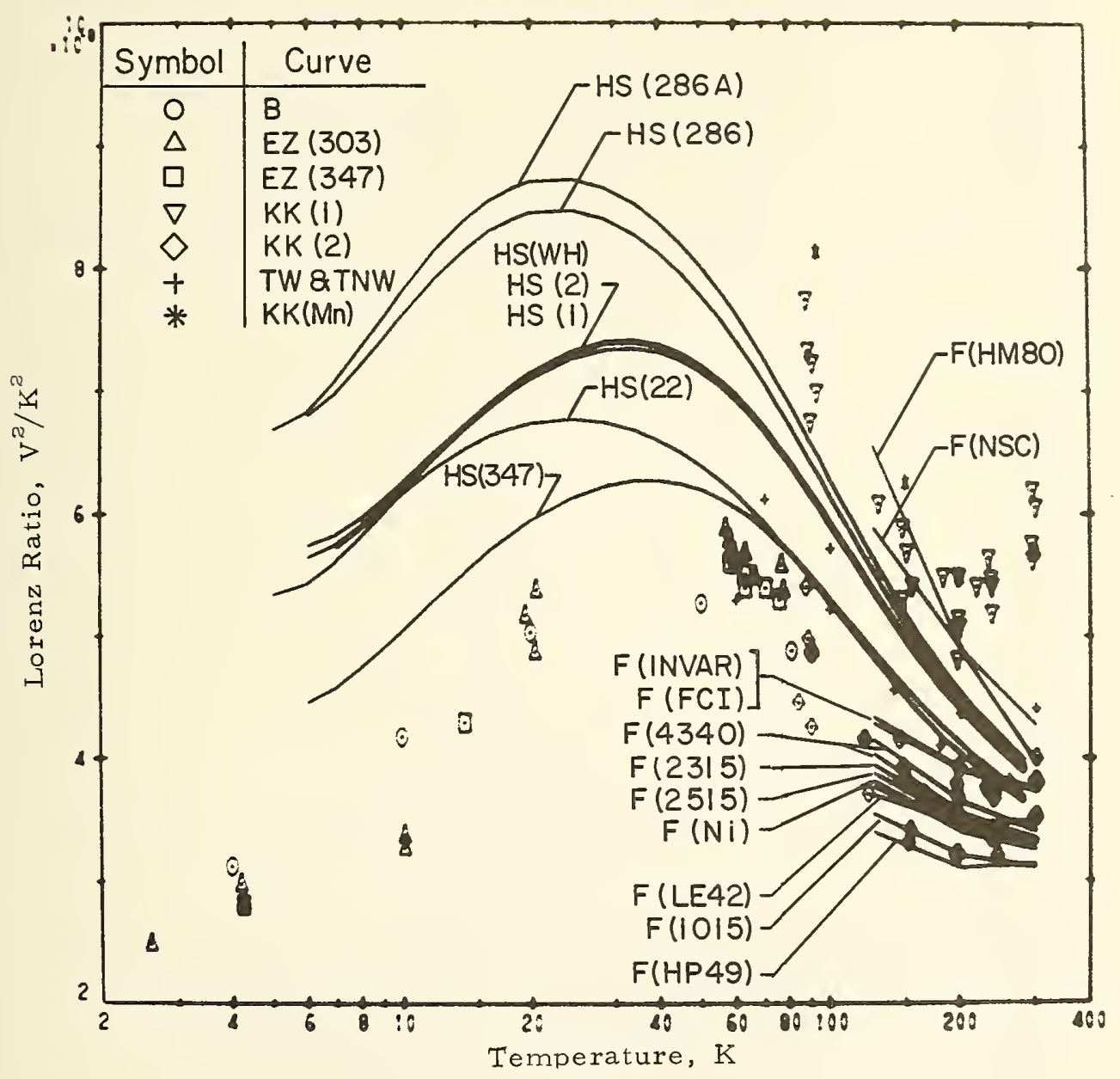

Figure 19. Lorenz ratio of stainless and alloy steels 


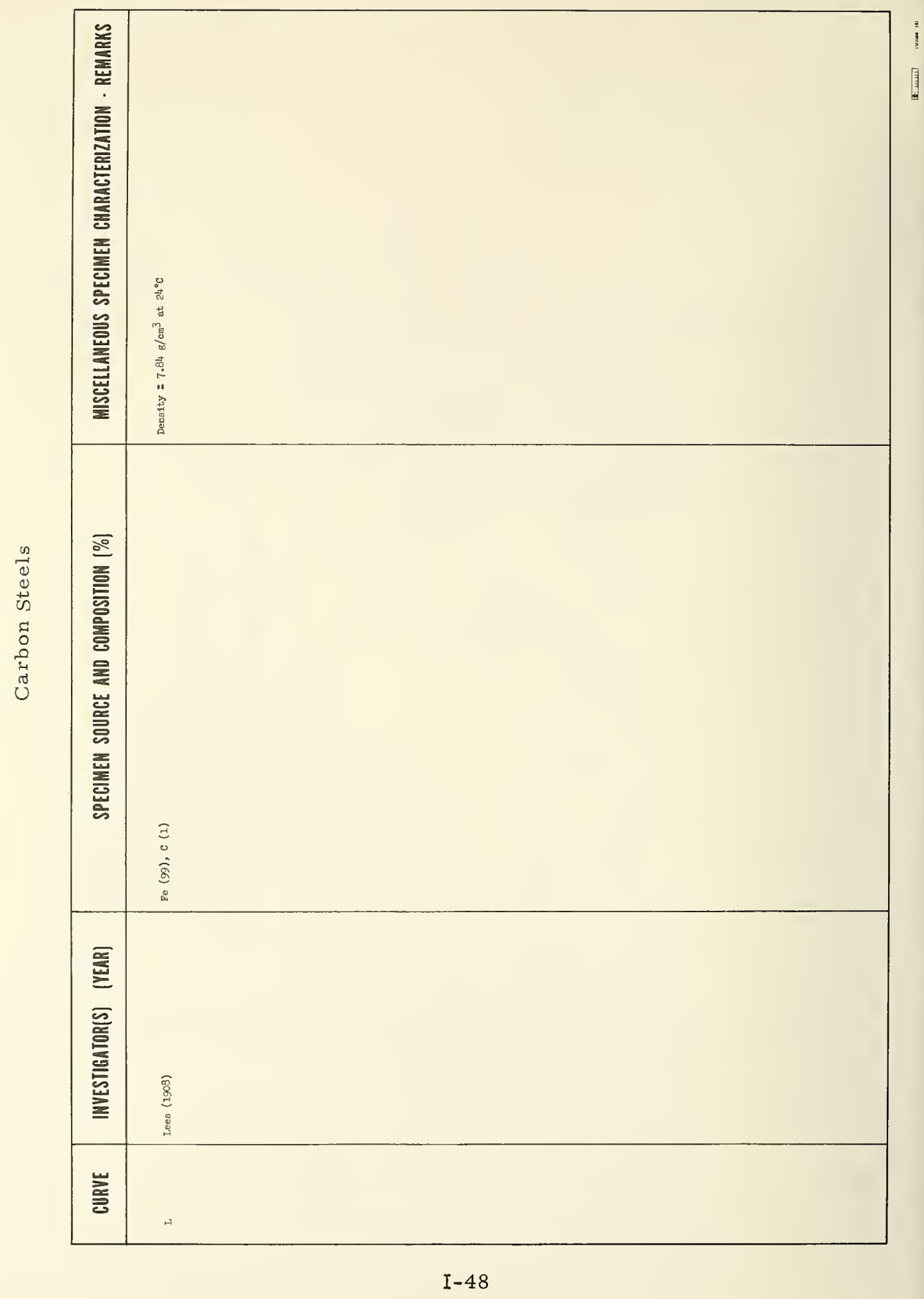




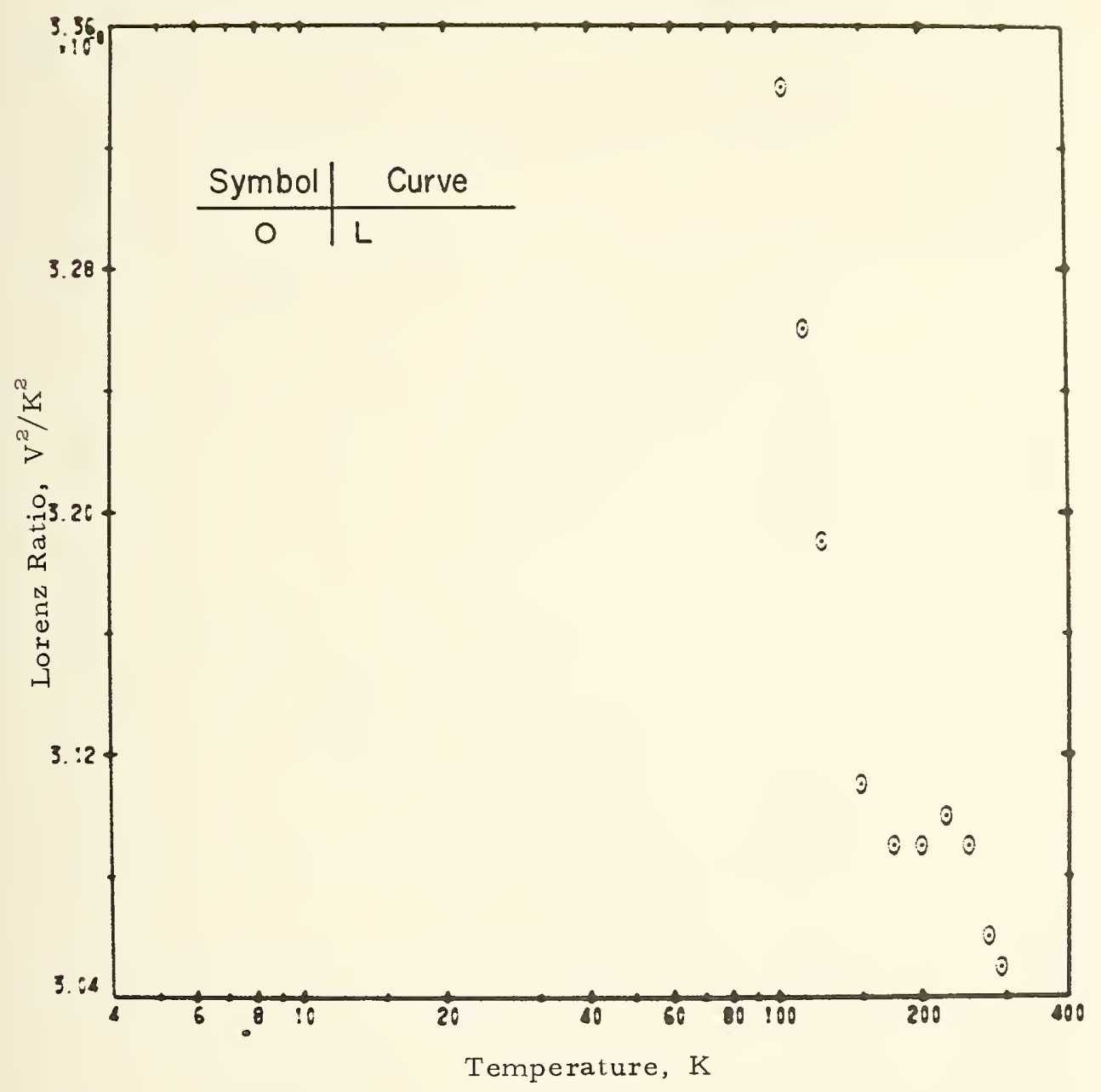

Figure 20. Lorenz ratio of carbon steels 


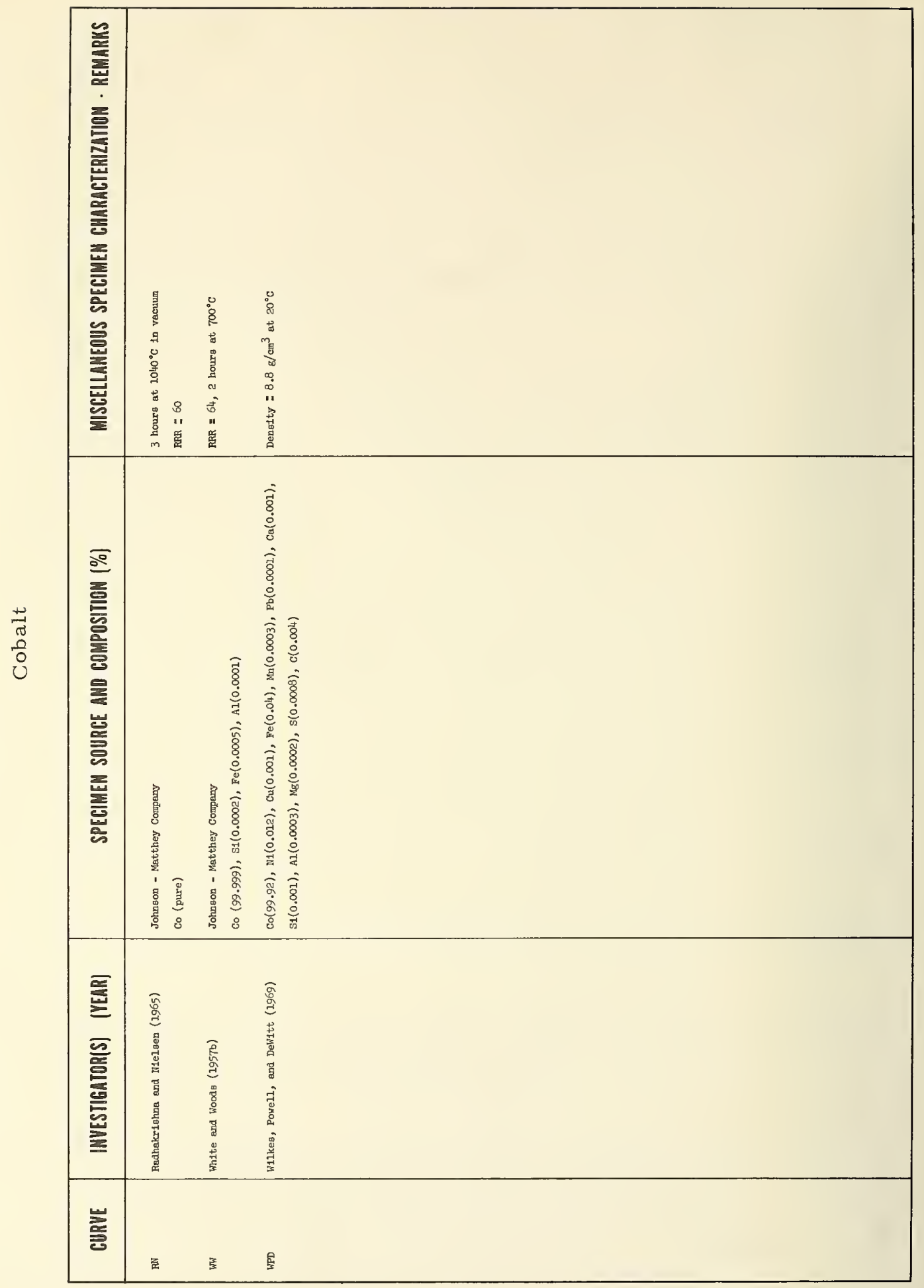




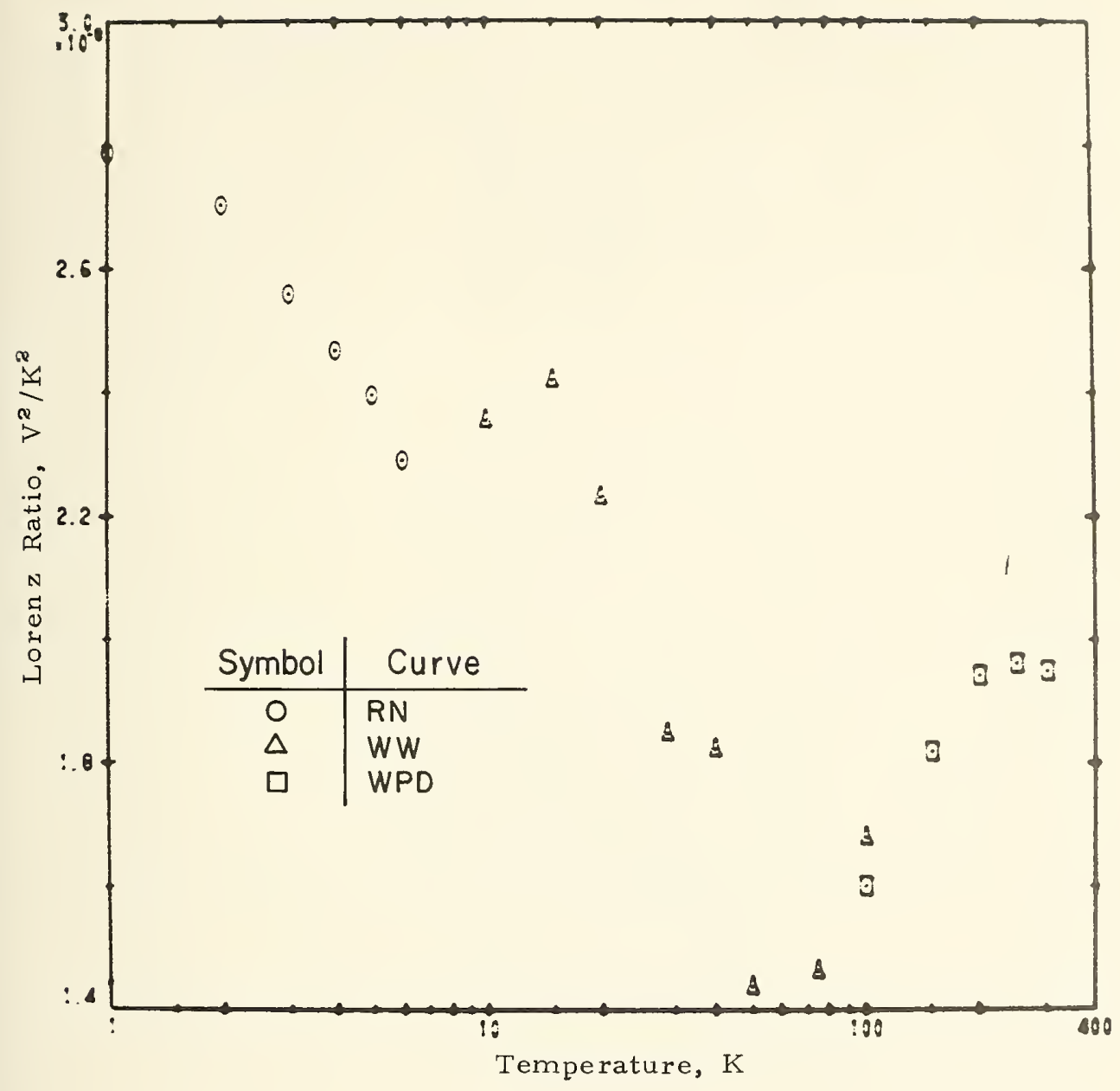

Figure 21. Lorenz ratio of cobalt 


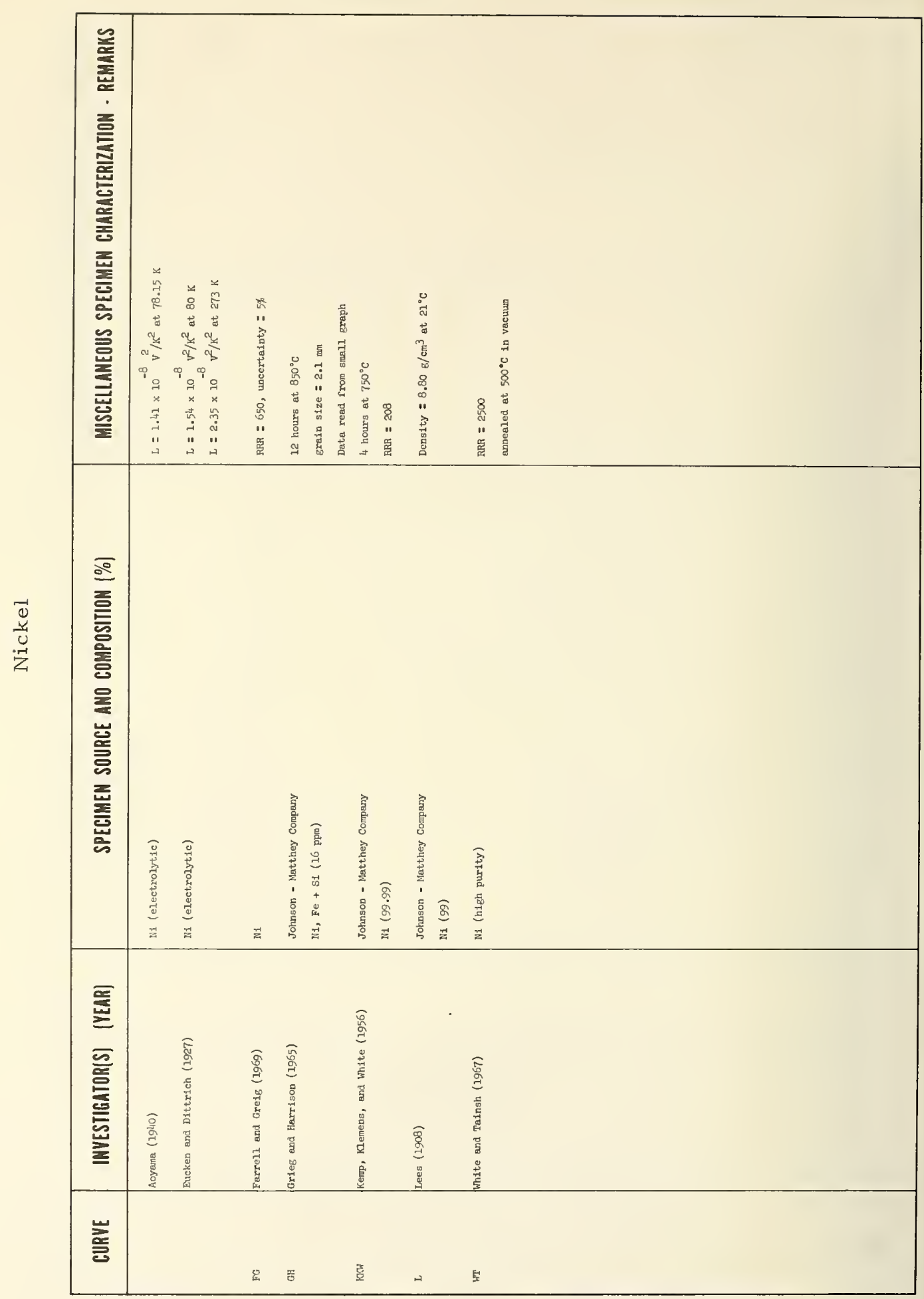

I- 52 


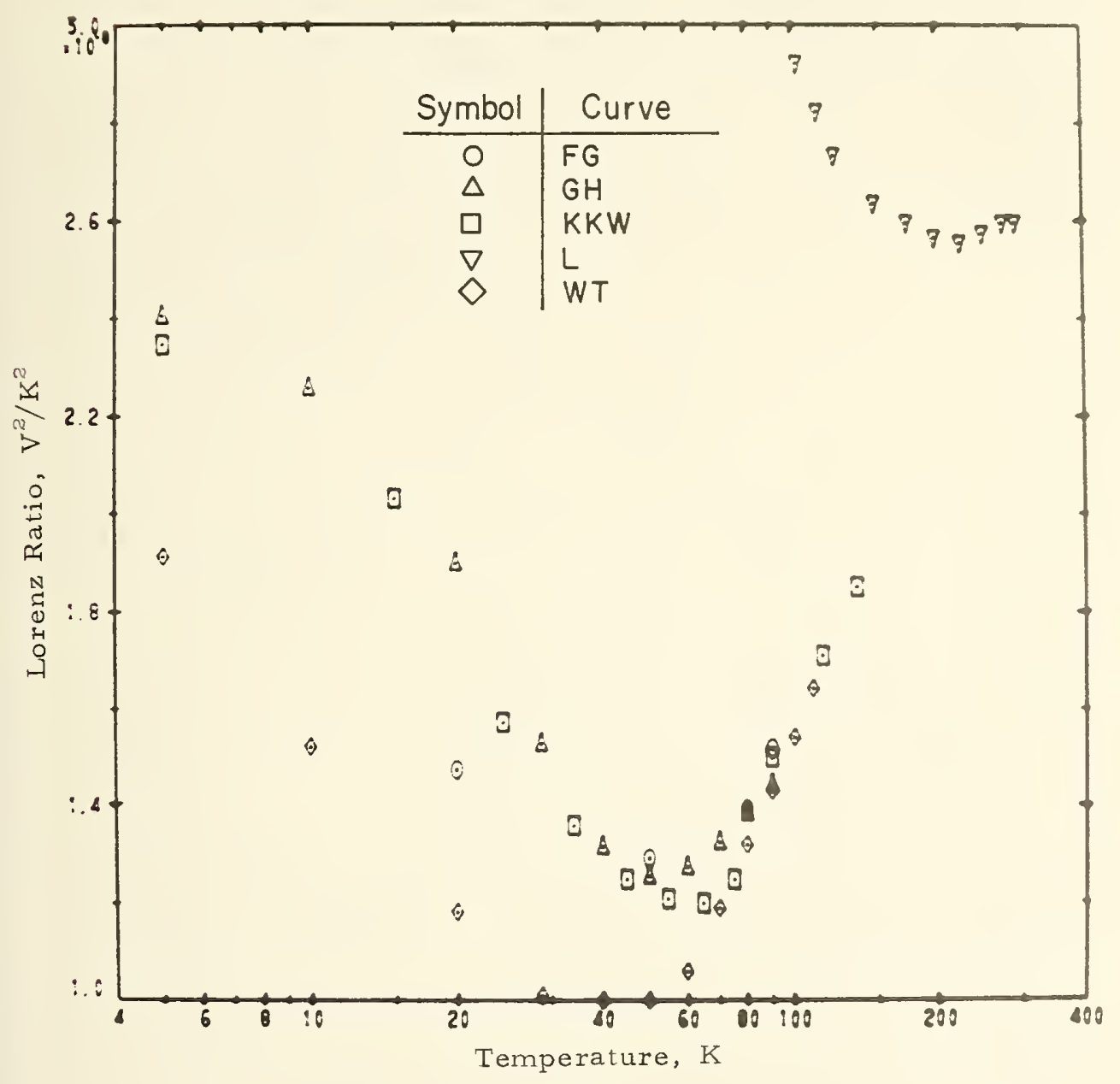

Figure 22. Lorenz ratio of nickel 


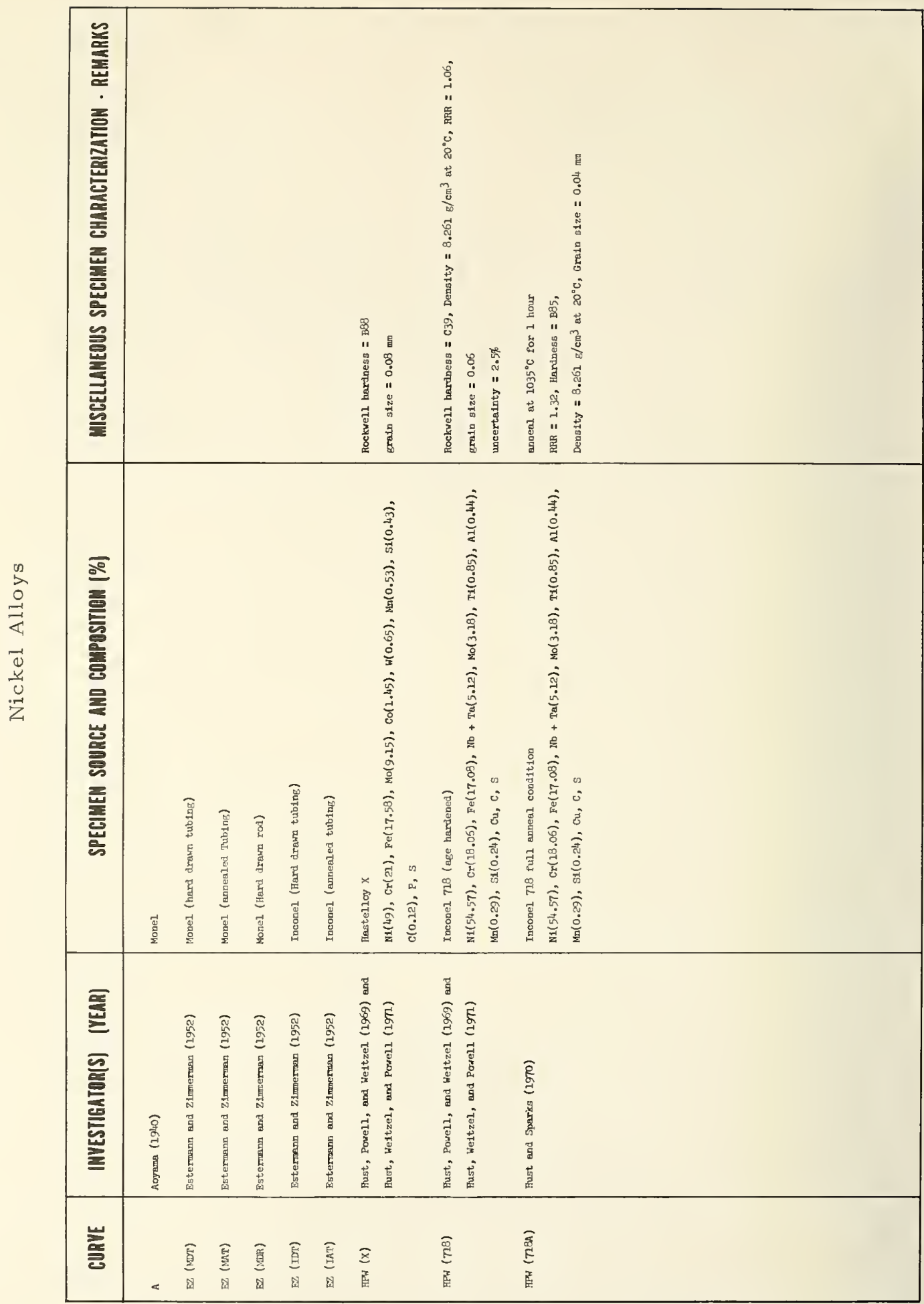




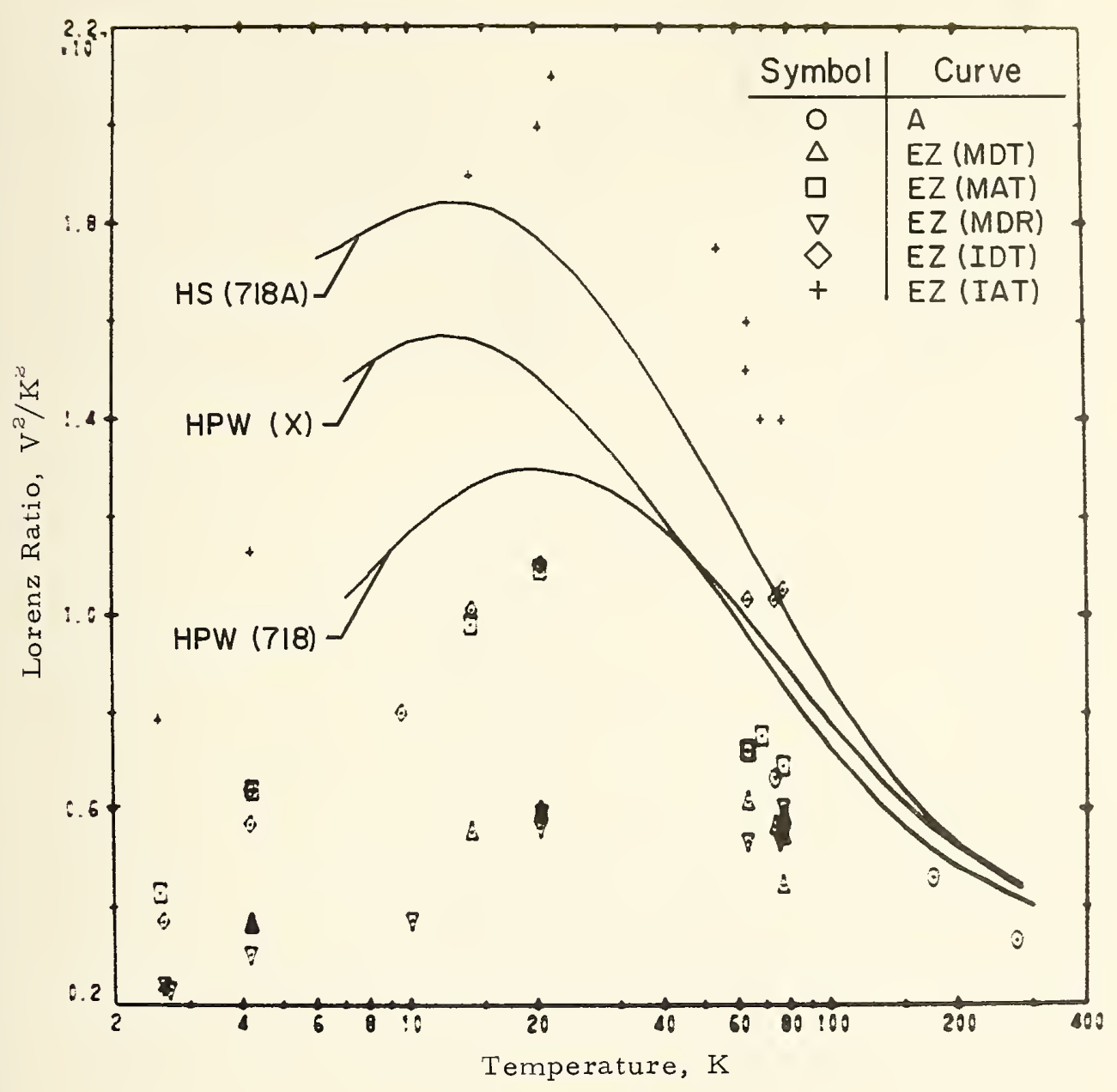

Figure 23. Lorenz ratio of nickel alloys 


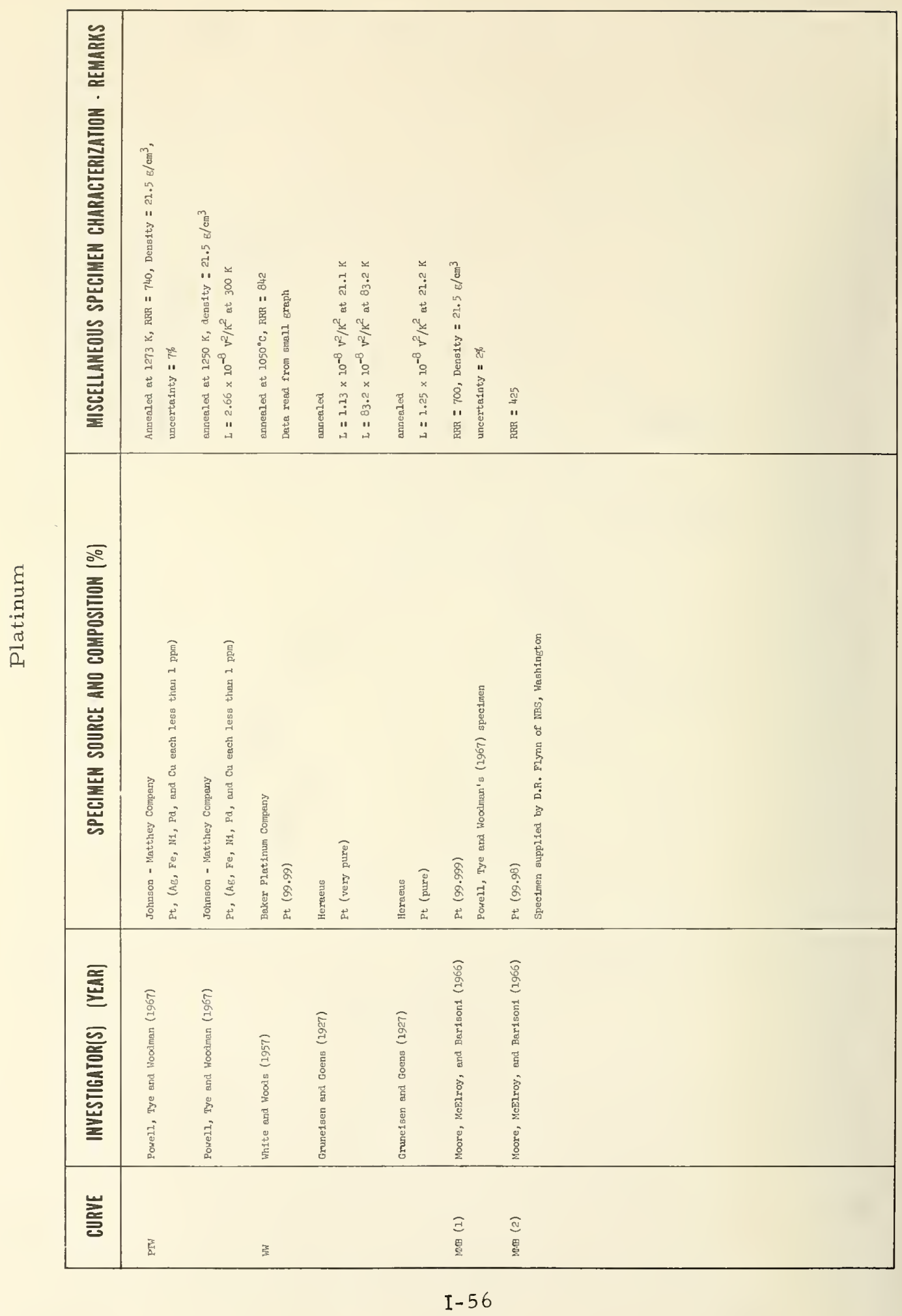




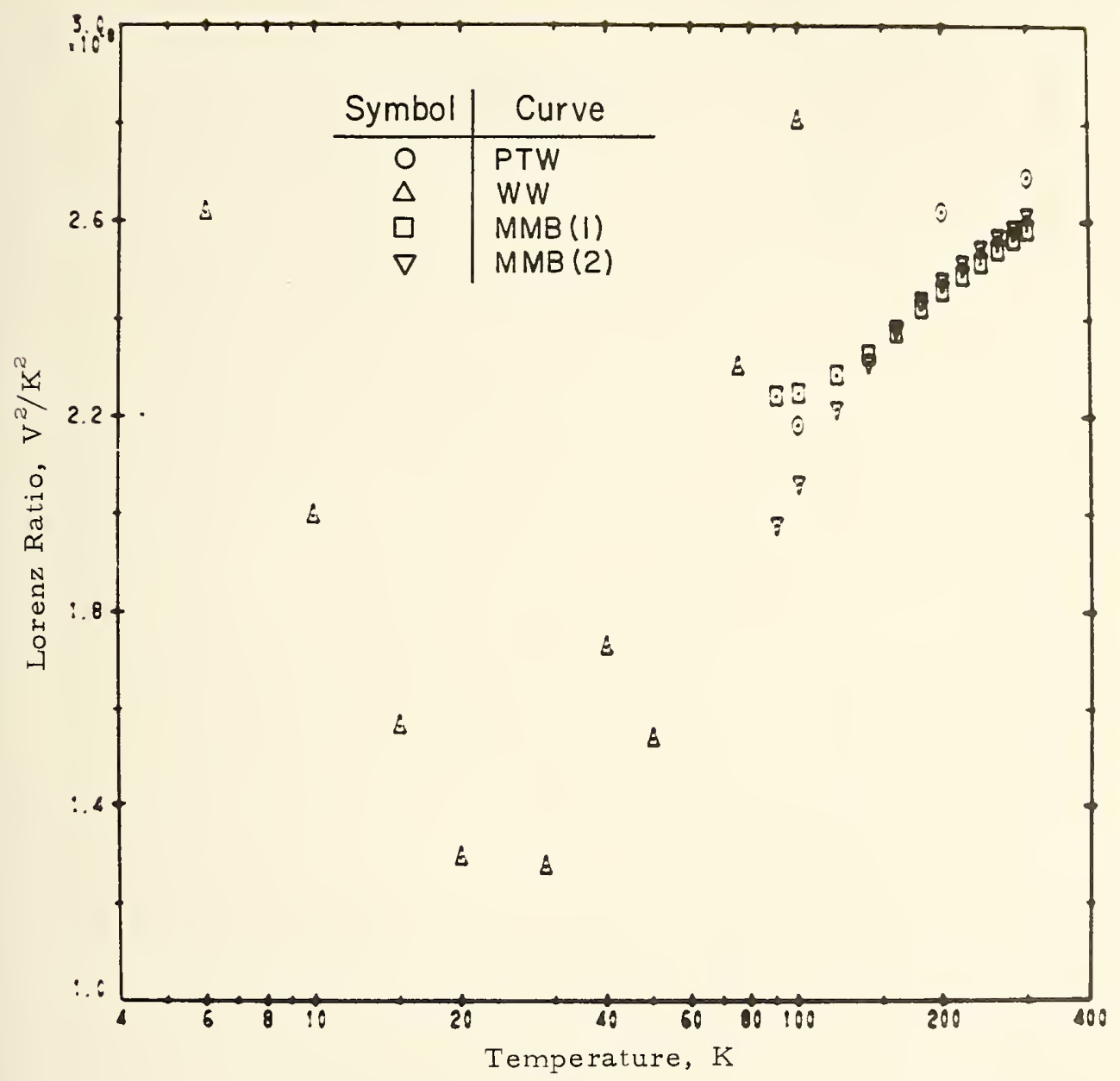

Figure 24. Lorenz ratio of platinum 


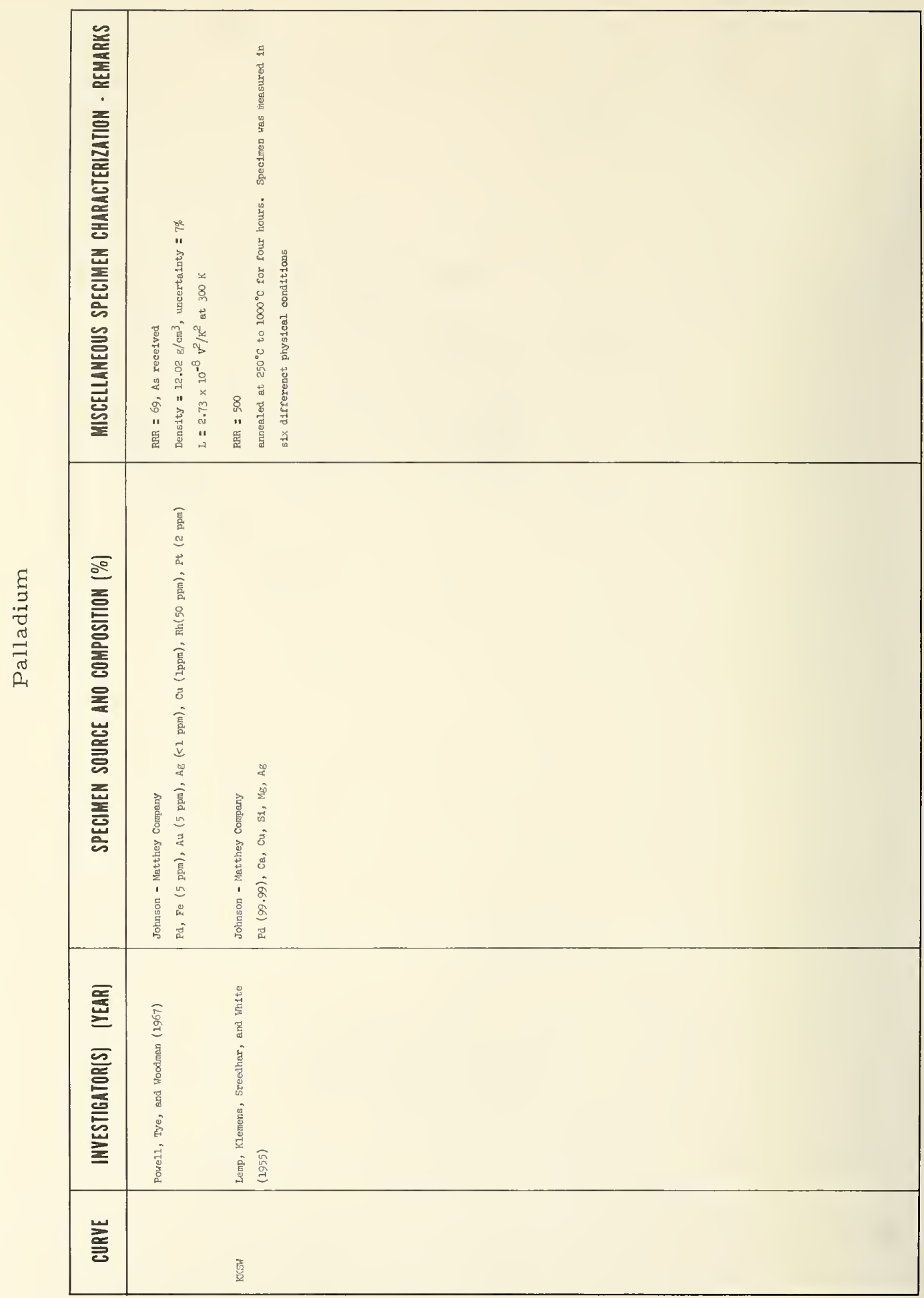




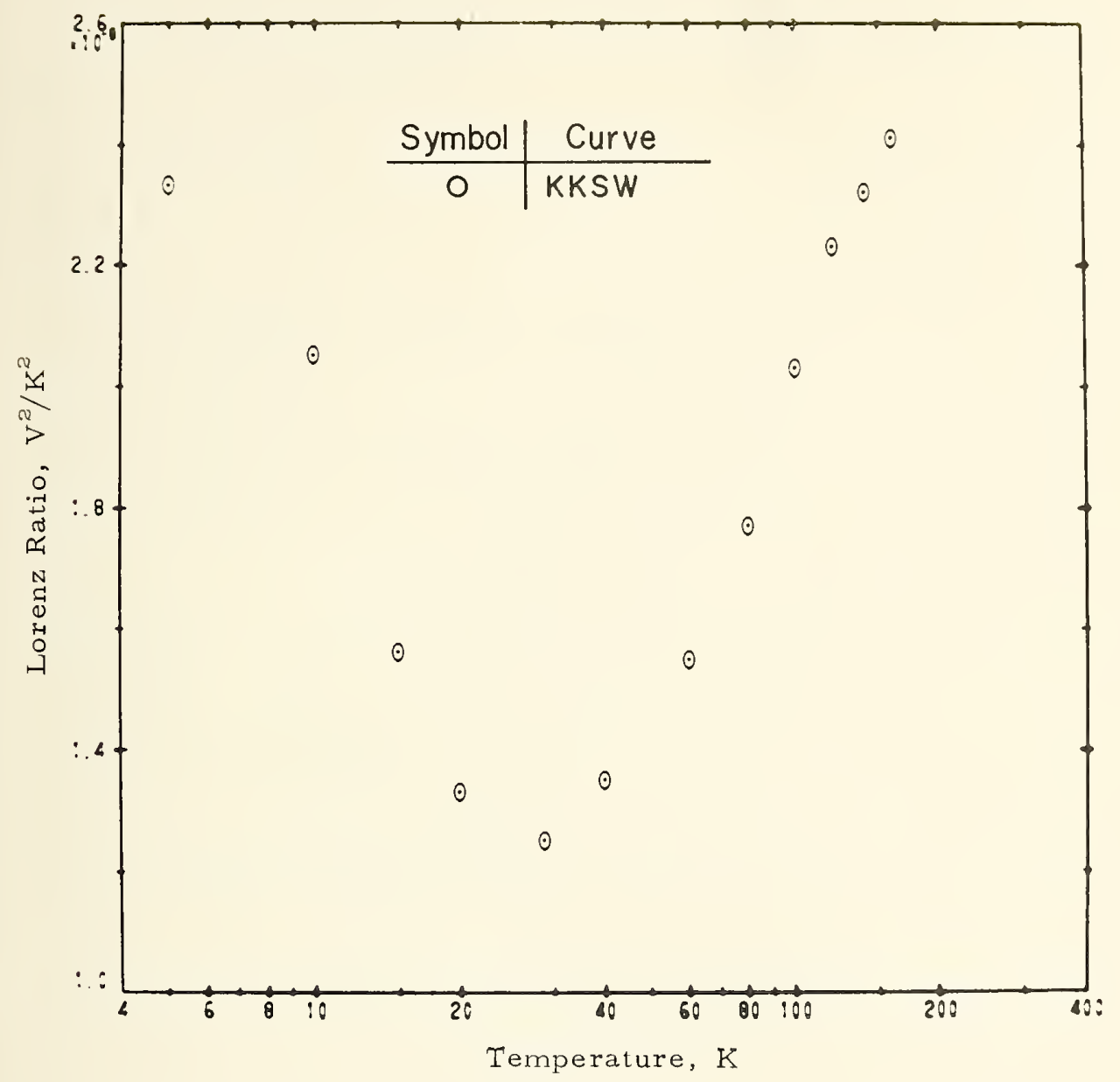

Figure 25. Lorenz ratio of palladium 
Beryllium . . . . . . . . . . . . . II- 2

Magnesium ................. . . . . . . 3

Aluminum . . . . . . . . . . . . . . II- 4

Aluminum Alloys . . . . . . . . . . . . II- 6

Lead and Tin ............ . . . . . II- 8

Gold . . . . . . . . . . . . . . . . . II- 9

Gold Cobalt Alloy . . . . . . . . . . . . . II-11

Silver . . . . . . . . . . . . II-12

Copper . . . . . . . . . . . . . . II-14

Copper Alloys (German Silver and Brass). . . . . II-15

Zinc and Cadmium . . . . . . . . . . . II-16

Scandium and Yttrium . . . . . . . . . II-17

Titanium, Hafnium, and Zirconium ........ II-18

Titanium Alloys............. . . II- 19

Tungsten ................... . . . . . 20

Molybdenum . . . . . . . . . . . II-22

Chromium . . . . . . . . . . . II-23

Manganese and Rhenium . . . . . . . . . II-25

Iron . . . . . . . . . . . . . . II-26

Stainless and Alloy Steels .............. II-30

Carbon Steels . . . . . . . . . . . II-37

Cobalt . . . . . . . . . . . . . II-37

Nickel . . . . . . . . . . . . . . II-38

Nickel Alloys ....... . . . . . . . II-39

Platinum . . . . . . . . . . . II-42

Palladium . . . . . . . . . . . II-42 


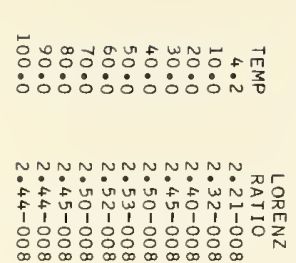

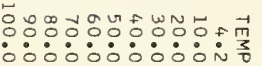

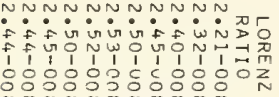

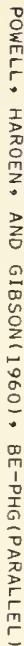

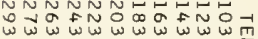

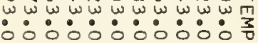

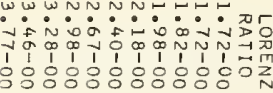

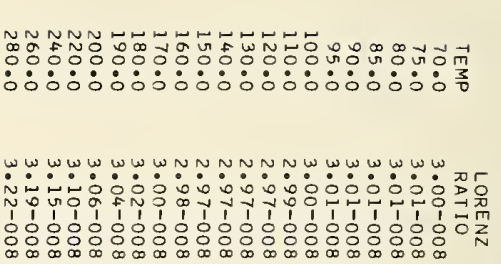

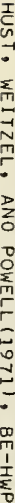

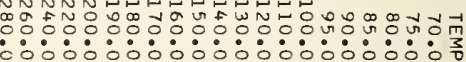

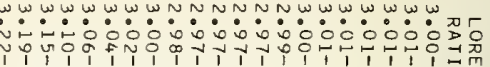

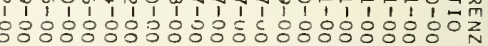

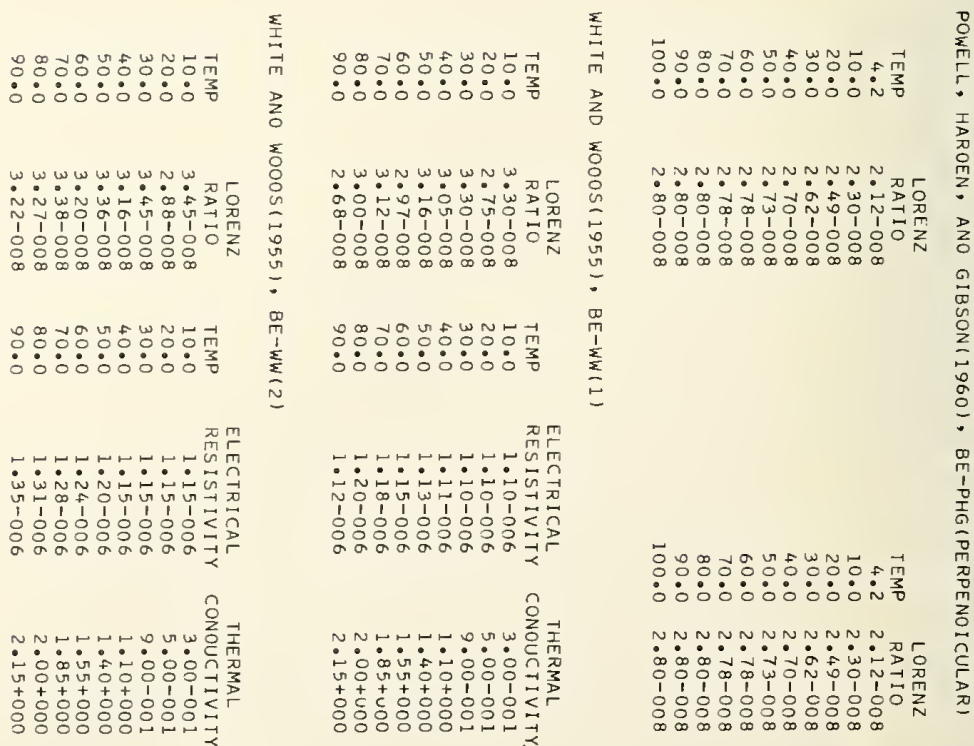


MAGNESIUM

SEE APPENDIX I - MAGNESIUM

II - 3 


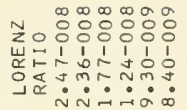

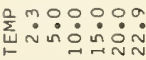

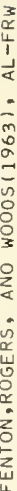

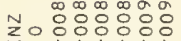

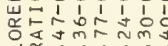

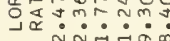

虽

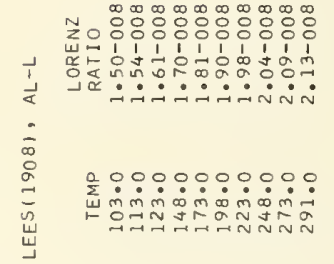

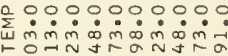
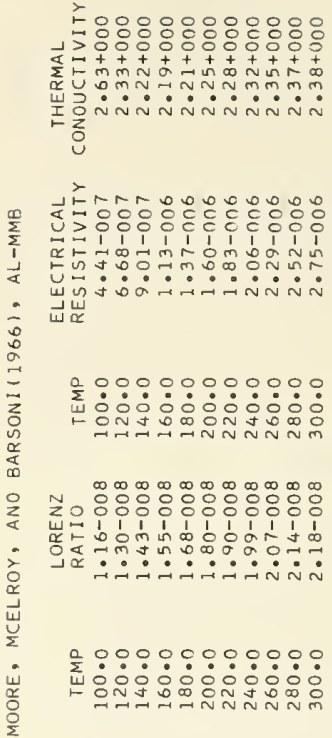

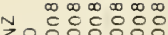

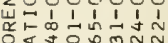
品:

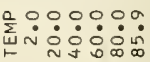

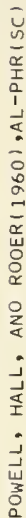

N

zo이인

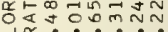

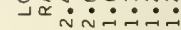

$\frac{2}{2}: 90909$

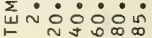

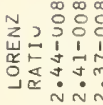

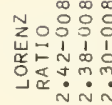

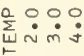

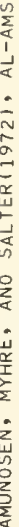

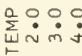

\section{Z̃o \\ 然安称 \\ 禹定}

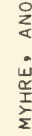

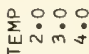

Z

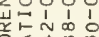

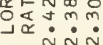

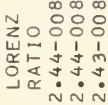

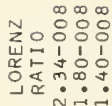

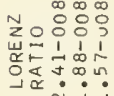

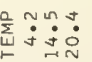

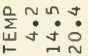

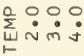

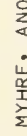

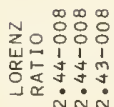

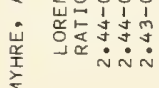

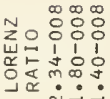

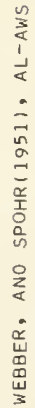

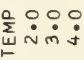

구에

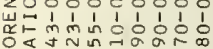

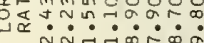

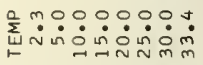

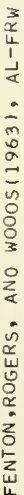

N $\infty^{\infty} \infty$ ara

zo 0000880

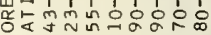

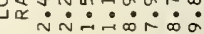

产 
$\underset{\square}{3}$

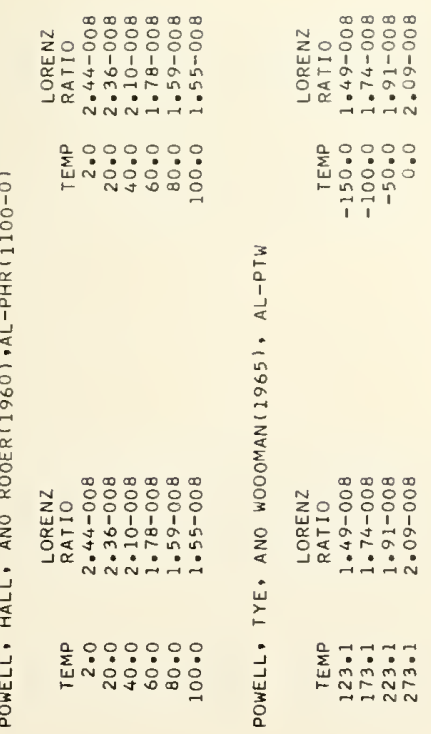


N L

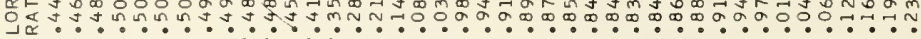

0

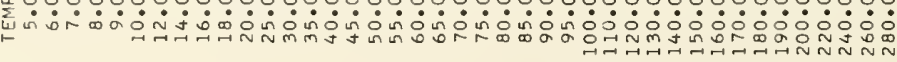

N Thipipipipipipipipipipipipip

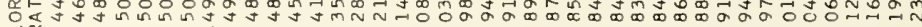

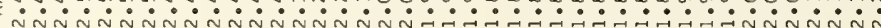

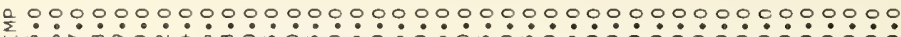

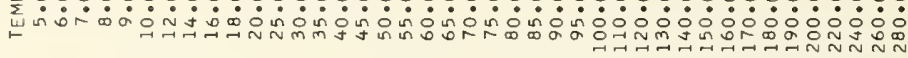

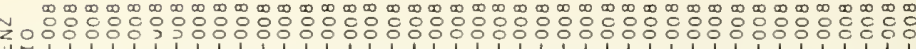

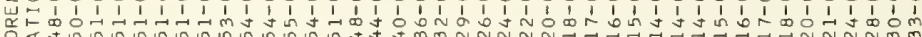

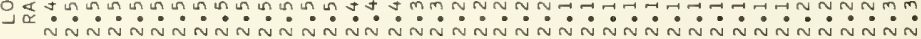

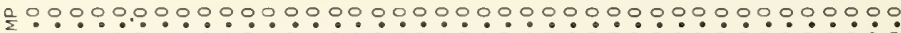

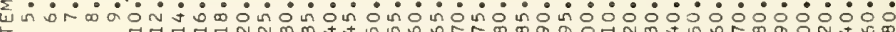
웅

No:

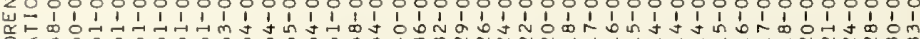

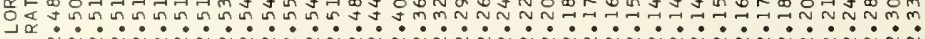

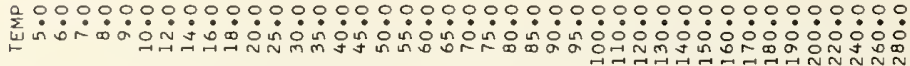




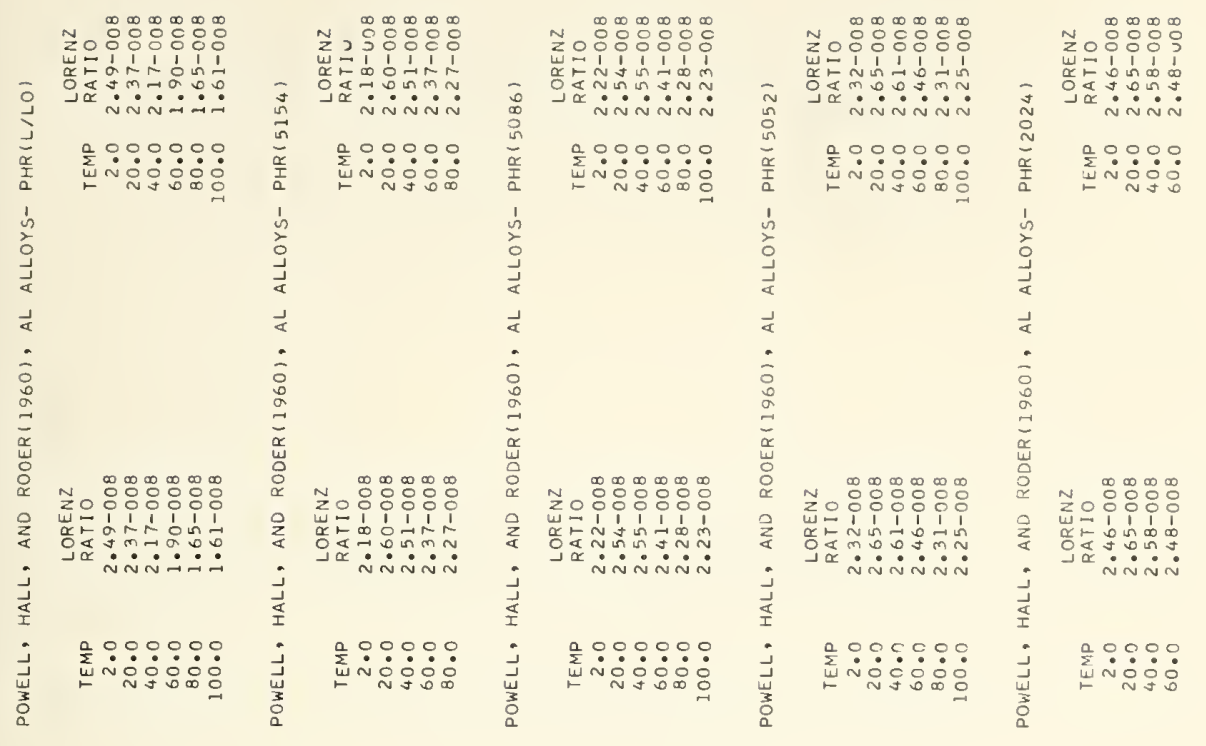

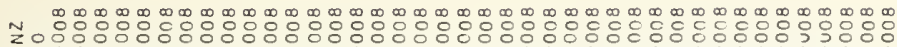

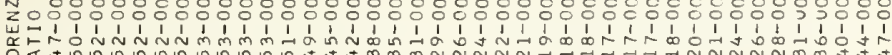

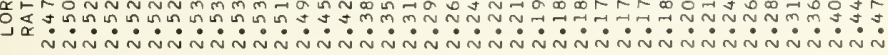

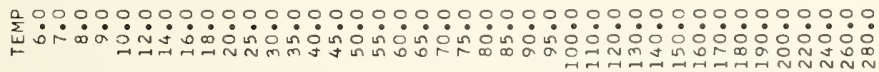

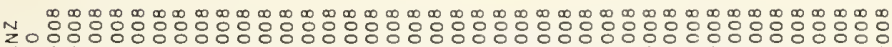

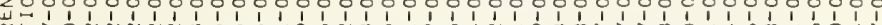

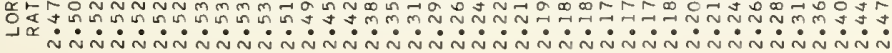

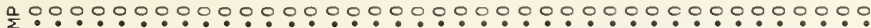

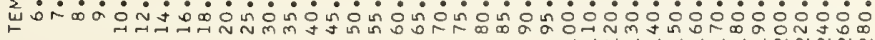




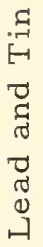

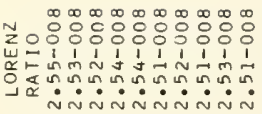

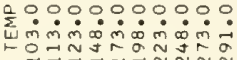

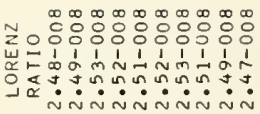

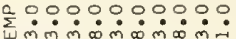

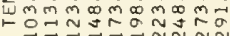

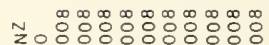

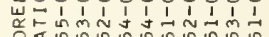

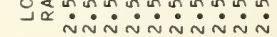

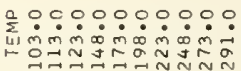

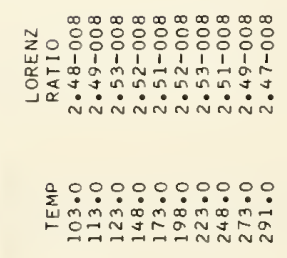


N

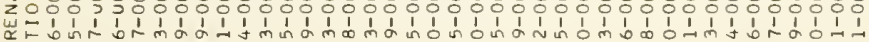

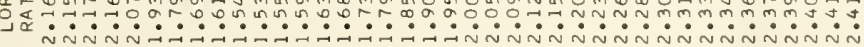

\section{a 0.0090000000090000000090090900000000000

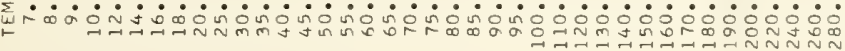

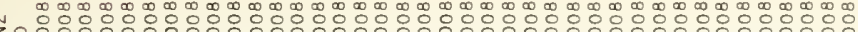

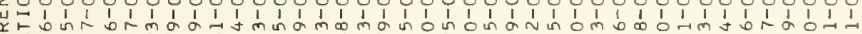
$\underset{\alpha}{\alpha}$

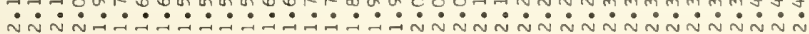

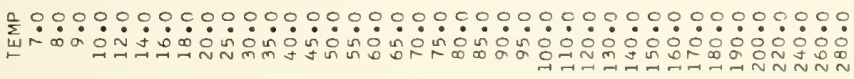

$N \quad 0^{\infty} 0^{\infty} 0^{\infty}$

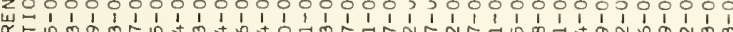

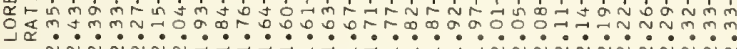

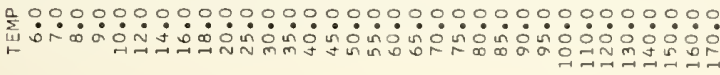

$\begin{array}{lll}\infty & \infty & \infty\end{array}$

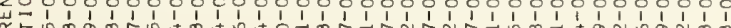

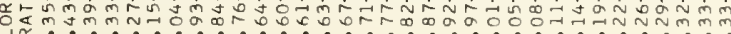

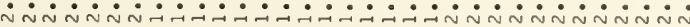

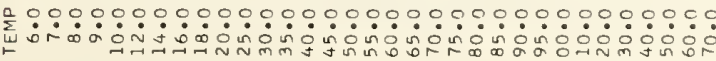



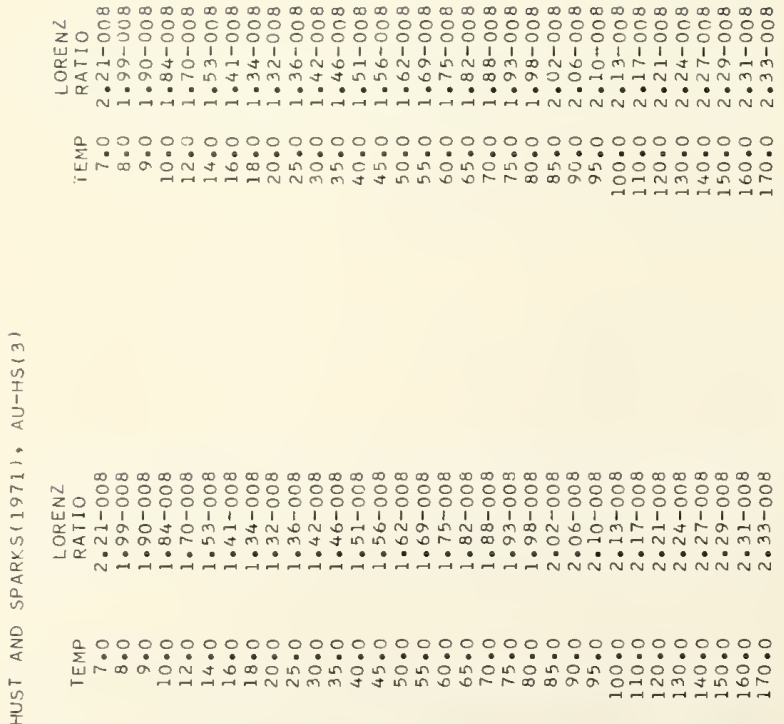


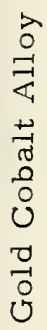

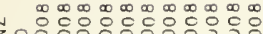

U.

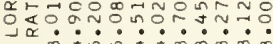

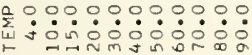

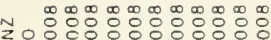

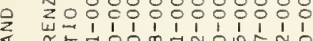

-

茨

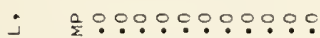

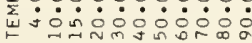




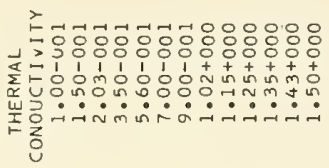

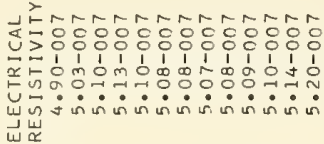

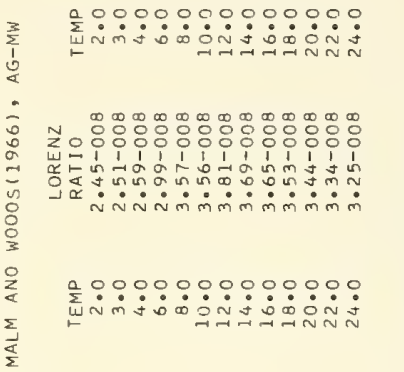

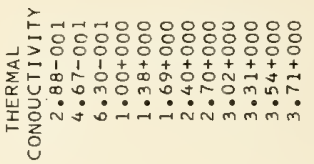

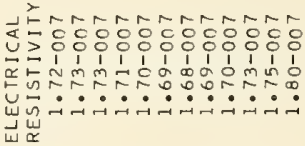

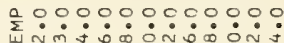

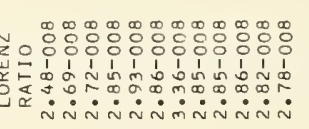

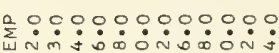

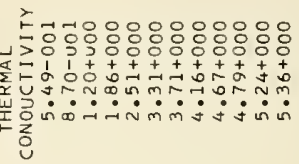

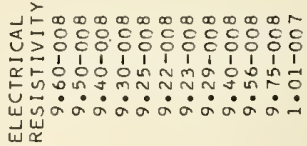

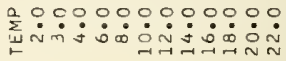

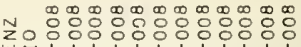

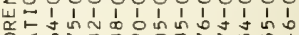
西:

$$
\text { nnninmininin }
$$

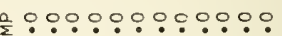

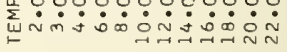

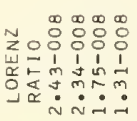

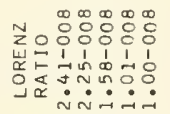

$\sum_{i=1}^{a} \sim: \because: 0:$$$
\frac{1}{\frac{1}{2}}
$$$$
\stackrel{1}{4}
$$$$
\begin{aligned}
& 3 \\
& 2 \\
& 5 \\
& -1 \\
& 0 \\
& 0 \\
& 0
\end{aligned}
$$

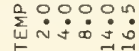

3

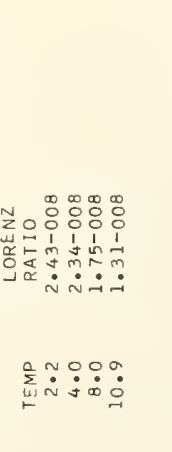

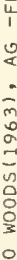

\section{N}

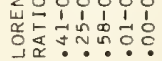

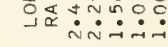

$\sum_{\text {แ }}^{\circ} \dot{\sim}: \dot{0} \dot{0}: \dot{0}$

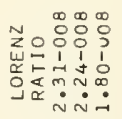

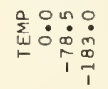
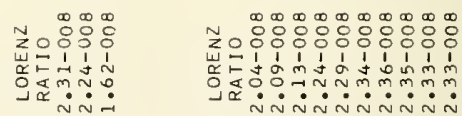

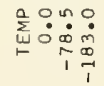

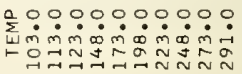

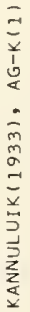

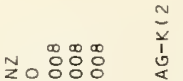

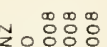

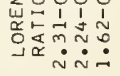

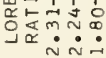

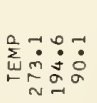

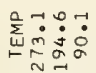

స̌

W

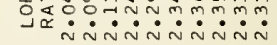

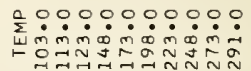


ن.

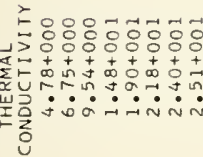

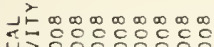

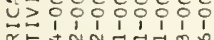

的ง

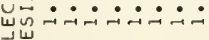

岀崫

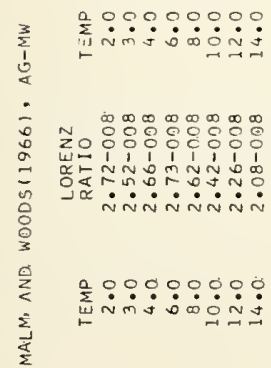




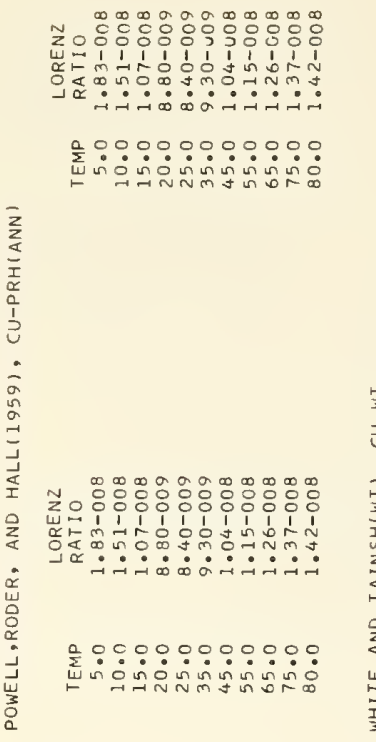

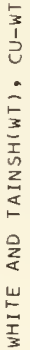

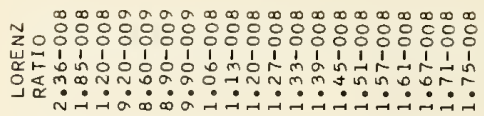

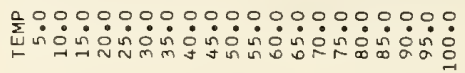
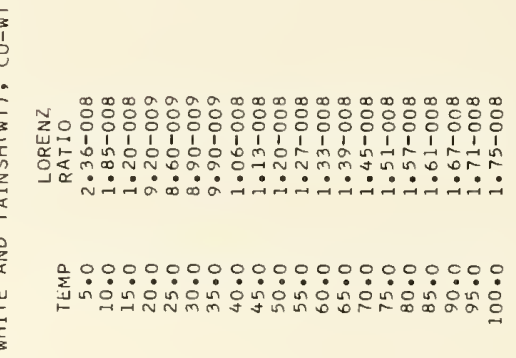

$\stackrel{1}{0}$
0
$\Omega_{1}$
0
0
0

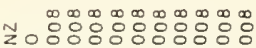

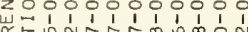

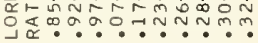

a $000: 000.00$

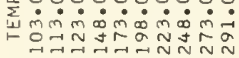

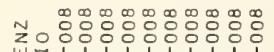

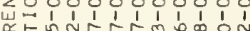

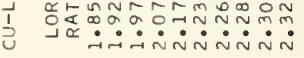

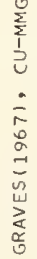

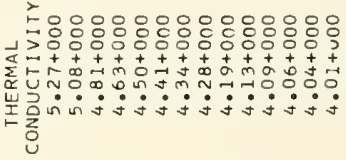

ح̃o̊

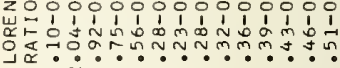

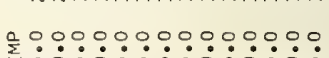

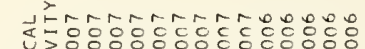

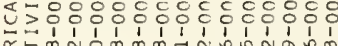

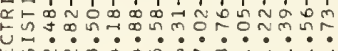

岀出

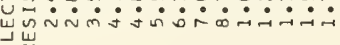

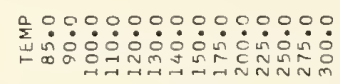

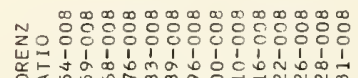

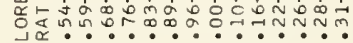

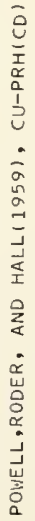

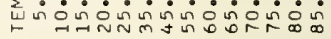

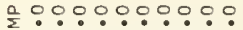

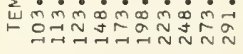

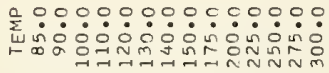

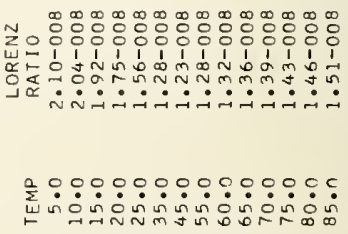


ㄴ.

$\psi$

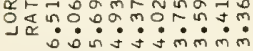

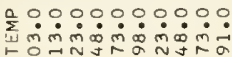

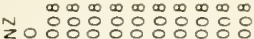

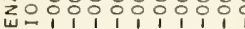

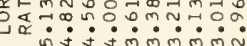

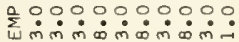

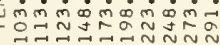

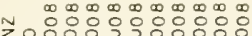

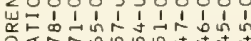

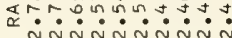

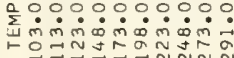

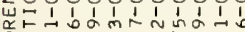

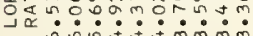

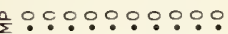

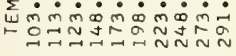

N

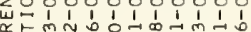

每

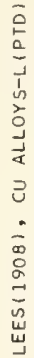

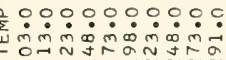

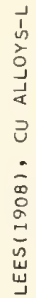

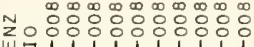

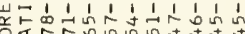
व

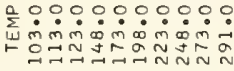

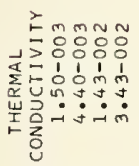

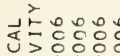

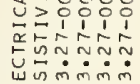

山ै山

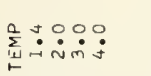

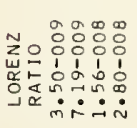

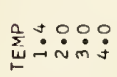

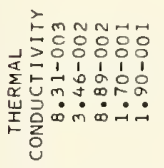

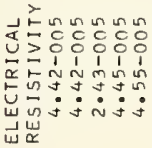

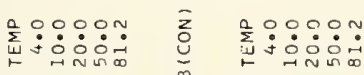

is

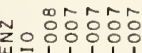

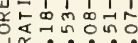

ฯ

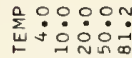

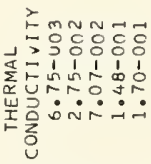

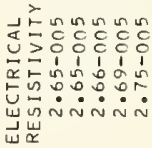

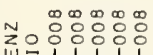

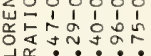

这地

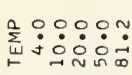

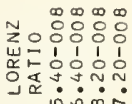

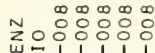

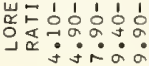

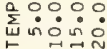

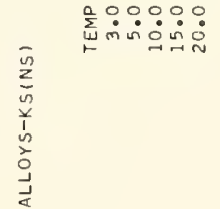

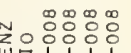

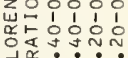

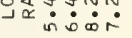

शे

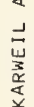

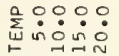

उ

衣

그용융요융

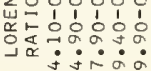

赵

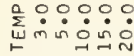




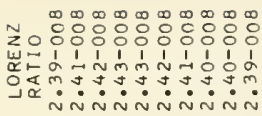

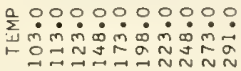

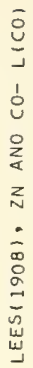

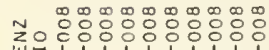

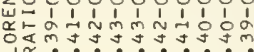

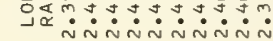

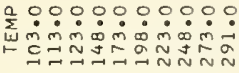

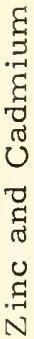

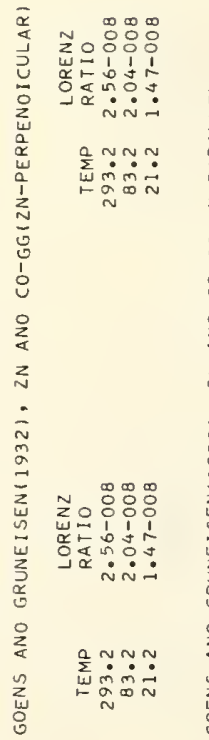

그용요유

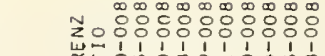

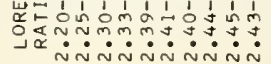

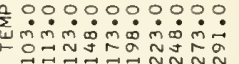

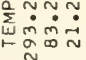

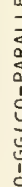

i

$\dot{N}$

a

ㄱ요요

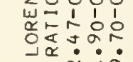

No

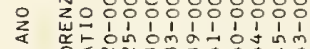

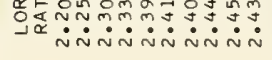

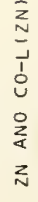

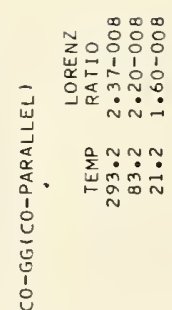

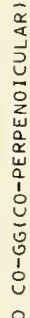

No

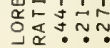

$\frac{a}{\Sigma} \simeq$ ?

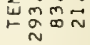

운

z

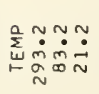

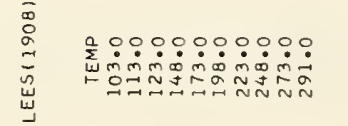

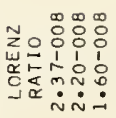

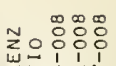

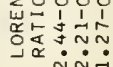

व

$\stackrel{0}{\stackrel{1}{*}}$

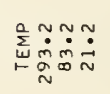

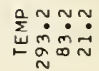


Scandium and Yttrium

ARAJS ANO COLVIN(1964), SC ANO Y-AC

\begin{tabular}{|c|c|c|c|}
\hline & LORENZ & & LORENZ \\
\hline TEMP & RATIO & TEMP & RATIO \\
\hline $4 \cdot 2$ & $7.00-008$ & $4 \cdot 2$ & $7.00-0 \cap 8$ \\
\hline $20 \cdot 0$ & $6.00-008$ & $20 \cdot 0$ & $6.00-008$ \\
\hline 40.0 & $4.20-008$ & 40.0 & $4.20-\cup 08$ \\
\hline $60 \cdot 0$ & $3 \cdot 30-008$ & 60.0 & $3.30-408$ \\
\hline $80 \cdot 0$ & $3 \cdot 10-008$ & $80 \cdot 0$ & $3.10-008$ \\
\hline $120 \cdot 0$ & $3.15-008$ & 120.0 & $3.15-008$ \\
\hline 160.0 & $3 \cdot 30-008$ & 160.0 & $3.30-008$ \\
\hline $200 \cdot 0$ & $3.60-008$ & 200.0 & $3.60-008$ \\
\hline 240.0 & $3.85-008$ & 240.0 & $3.85-008$ \\
\hline 280.0 & $4.35-008$ & 280.0 & $4 \cdot 35-008$ \\
\hline $300 \cdot 0$ & $4.65-008$ & 300.0 & $4.65-008$ \\
\hline
\end{tabular}

TAMARIN, CHUPRIKOV, ANO SHALYT(1969), SC ANO Y- TCS(PARALLEL)

\begin{tabular}{|c|c|c|c|}
\hline & LORENZ & & LORENZ \\
\hline TEMP & RATIO & TEMP & RATIO \\
\hline $5 \cdot 0$ & $4.20-008$ & 5.0 & $4 \cdot 20-008$ \\
\hline 10.0 & $4.70-008$ & 10.0 & $4.70-008$ \\
\hline 17.0 & $5.00-008$ & 17.0 & $5.00-008$ \\
\hline $20 \cdot 0$ & $4.90-008$ & 20.0 & $4.90-008$ \\
\hline $30 \cdot 0$ & $4.50-008$ & 30.0 & $4.50-008$ \\
\hline 40.0 & $4.10-008$ & 40.0 & $4.10-008$ \\
\hline 50.0 & $3.95-008$ & 50.0 & $3.95-008$ \\
\hline $60 \cdot 0$ & $3.90-008$ & 60.0 & $3.90-008$ \\
\hline $70 \cdot 0$ & $3 \cdot 80-008$ & $70 \cdot 0$ & $3.80-008$ \\
\hline 80.0 & $3.77-008$ & $80 \cdot 0$ & $3.77-008$ \\
\hline 90.0 & $3.75-008$ & 90.0 & $3.75-008$ \\
\hline $100 \cdot 0$ & $3 \cdot 72-008$ & $100 \cdot 0$ & $3.72-008$ \\
\hline 110.0 & $3.70-008$ & 110.0 & $3.70-008$ \\
\hline $120 \cdot 0$ & $3.68-008$ & 120.0 & $3.68-008$ \\
\hline $130 \cdot 0$ & $3 \cdot 67-008$ & 130.0 & $3.67-008$ \\
\hline 140.0 & $3.66-008$ & 140.0 & $3.66-008$ \\
\hline $150 \cdot 0$ & $3.65-008$ & 150.0 & $3.65-008$ \\
\hline
\end{tabular}

TAMARIN, SHUPRIKOV, ANO SHALYT(1969), SC ANO Y- TCS PERPENOICULAR)

$\begin{array}{rlrc} & \text { LORENZ } & & \text { LORENZ } \\ \text { TEMP } & \text { RATIO } & \text { TEMP } & \text { RATIO } \\ 5.0 & 3.00-008 & 5.0 & 3.00-008 \\ 10.0 & 3.05-008 & 10.0 & 3.05-\cup 08 \\ 14.0 & 3.10-008 & 14.0 & 3.10-008 \\ 20.0 & 3.00-008 & 20.0 & 3.00-008 \\ 30.0 & 2.47-008 & 30.0 & 2.47-008 \\ 40.0 & 2.00-008 & 40.0 & 2.00-008 \\ 50.0 & 1.95-008 & 50.0 & 1.95-008 \\ 60.0 & 1.90-008 & 60.0 & 1.90-008 \\ 70.0 & 1.95-008 & 70.0 & 1.95-008 \\ 80.0 & 2.00-008 & 80.0 & 2.00-008 \\ 100.0 & 2.03-008 & 100.0 & 2.03-008 \\ 120.0 & 2.47-008 & 120.0 & 2.47-008 \\ 140.0 & 2.50-008 & 140.0 & 2.50-008 \\ 150.0 & 2.53-008 & 150.0 & 2.53-008\end{array}$




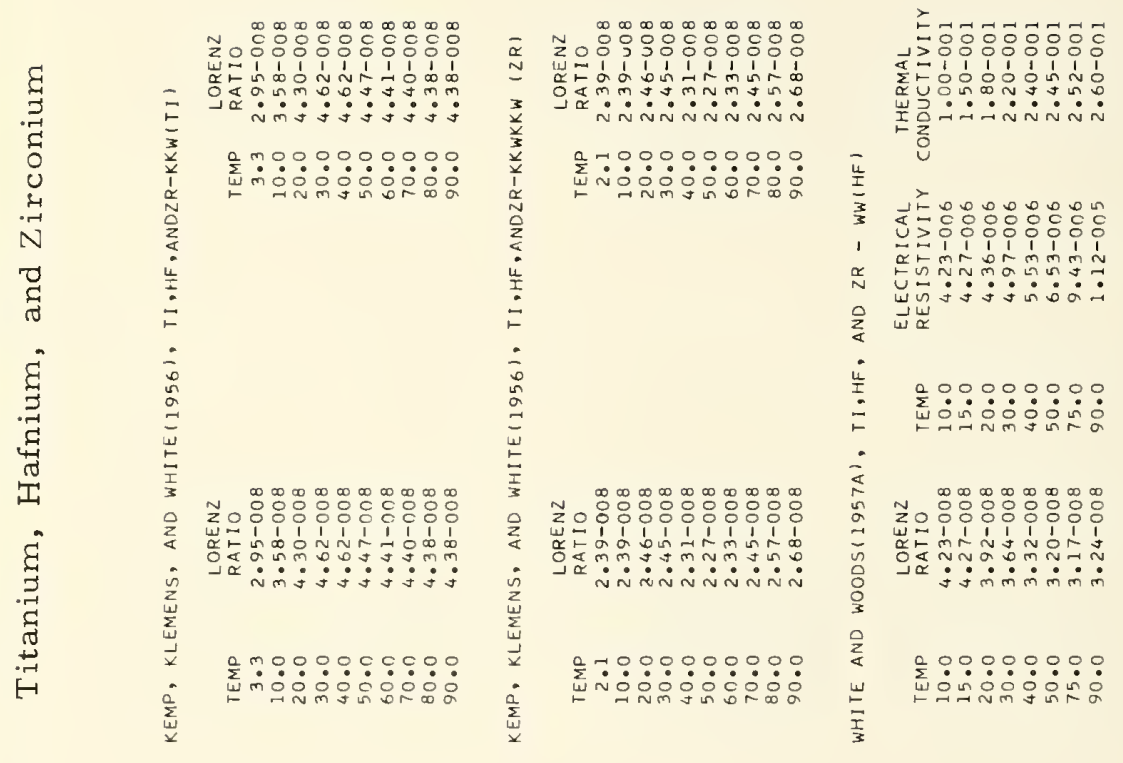


a

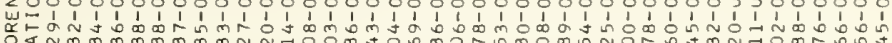

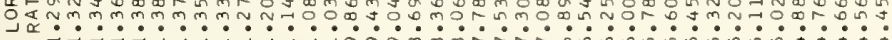

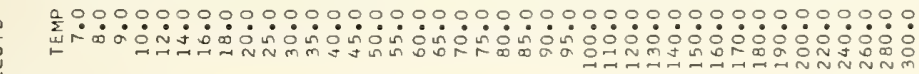

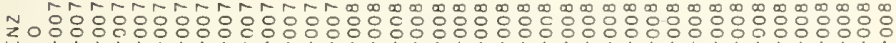
ZOM

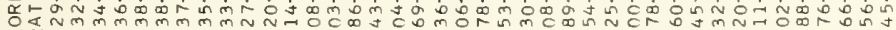

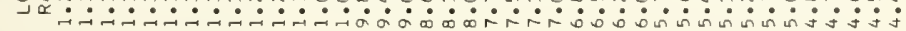

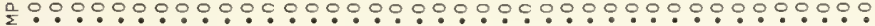

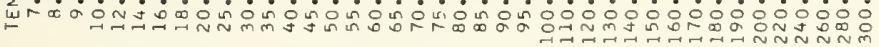




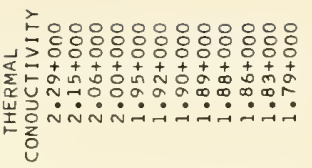

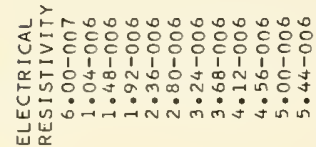

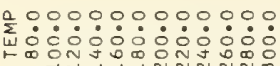

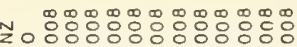

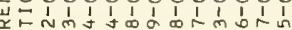

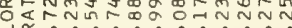

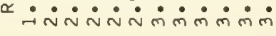

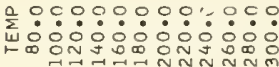

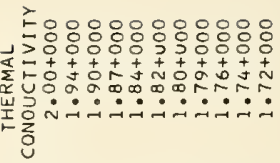

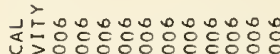

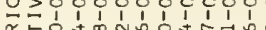

는ำ 岃出 $山 \propto$

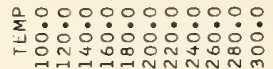

N

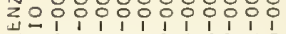

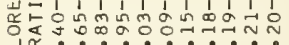

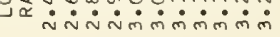

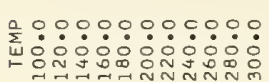

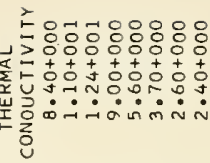

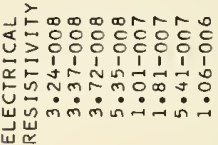

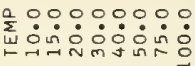
No

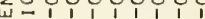

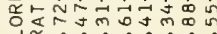

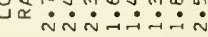

产.0.0.0.:.

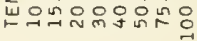

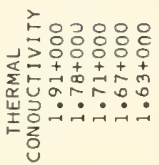

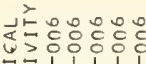

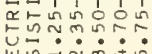

山ै山 또

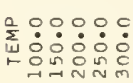

ㄱo:요요요

피눈

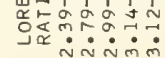

$r$

a

욱

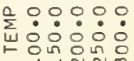

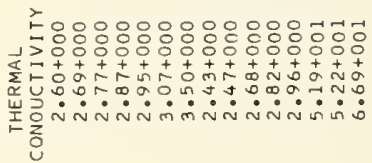

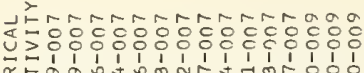

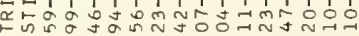

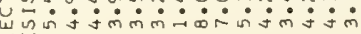

山ै

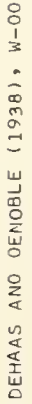

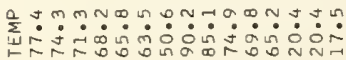

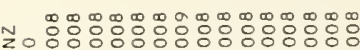

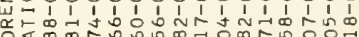

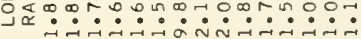

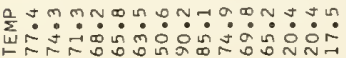

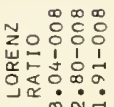

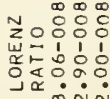

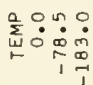

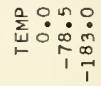

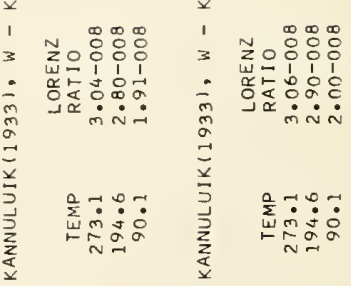




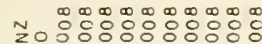

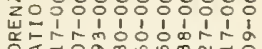

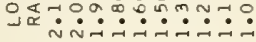

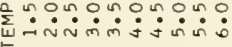

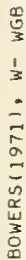

웅

¿

$\frac{\vec{x}}{8}$

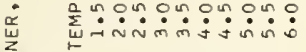

₹

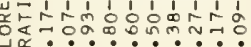

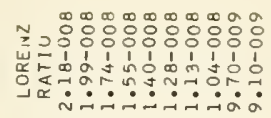

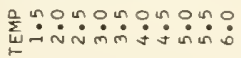

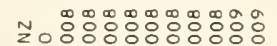

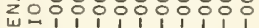

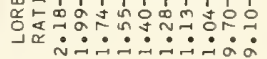

离

$\frac{1}{3}$

立

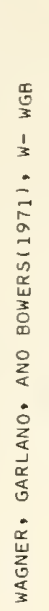

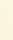

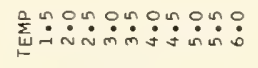

حै

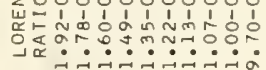

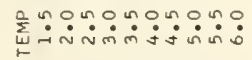

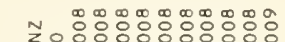

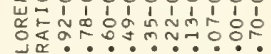

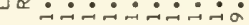

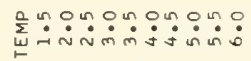

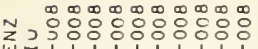

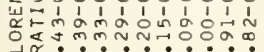

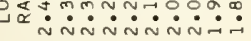

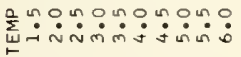

$\frac{0}{3}$

$\frac{1}{3}$

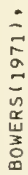

올

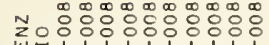

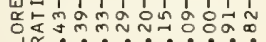

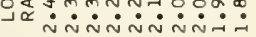

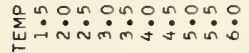

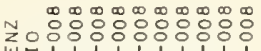

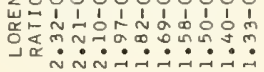

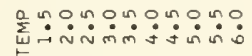

Z̃.

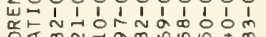

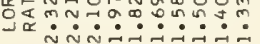
(1)

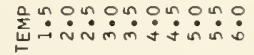

\begin{tabular}{l}
$\infty$ \\
N \\
Z \\
\hline
\end{tabular}

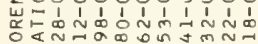

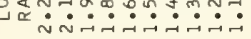

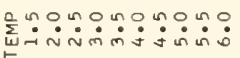

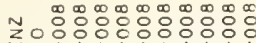
w. 范造

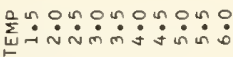


Molybdenum

BACKLUNO (1967), MO - B

$\begin{array}{rcccc}\text { IEMP } & \text { LORENZ } & \text { RLECTRICAL } & \text { THERMAL } \\ 100.0 & 1.74-008 & 100.0 & 1.05-006 & 1.66+000 \\ 150.0 & 2.24-008 & 150.0 & 2.30-006 & 1.46+000 \\ 200 \cdot 0 & 2.36-008 & 200 \cdot 0 & 3.35-006 & 1.41+000 \\ 250.0 & 2.53-008 & 250.0 & 4.55-006 & 1.39+000 \\ 300.0 & 2.54-008 & 300.0 & 5.60-006 & 1.36+000\end{array}$

KANNULUIK(1933), MO- K(1)

$\begin{array}{rl} & \text { LORENZ } \\ \text { TEMP } & \text { RAT IO } \\ 273.1 & 2.63-008 \\ 194.6 & 2.38-008 \\ 90.1 & 1.94-008\end{array}$

$\begin{array}{rl}\text { TEMP } & \text { RORENZ } \\ 0.0 & 2.63-008 \\ -78.5 & 2.38-008 \\ -183.0 & 1.94-008\end{array}$

KANNULUIK(1933), MO- K(2)

$\begin{array}{rl} & \text { LORENZ } \\ \text { TEMP } & \text { RATIO } \\ 273.1 & 2.61-008 \\ 194.6 & 2.35-008 \\ 90.1 & 1.76-008\end{array}$

$\begin{array}{rc} & \text { LORENZ } \\ \text { TEMP } & \text { RAT } 10 \\ 0.0 & 2.61-008 \\ -78.5 & 2.35-008 \\ -1.83 .0 & 1.76-008\end{array}$



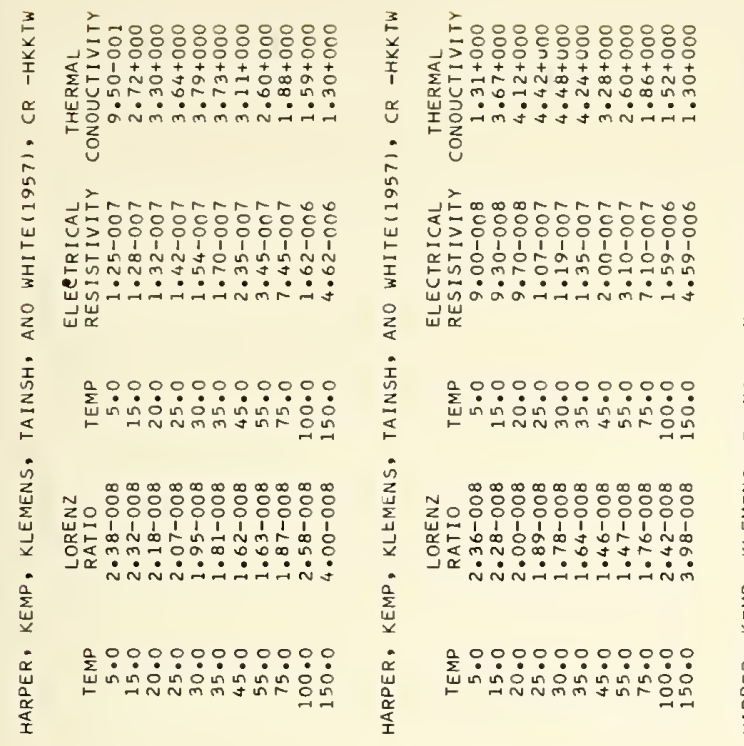

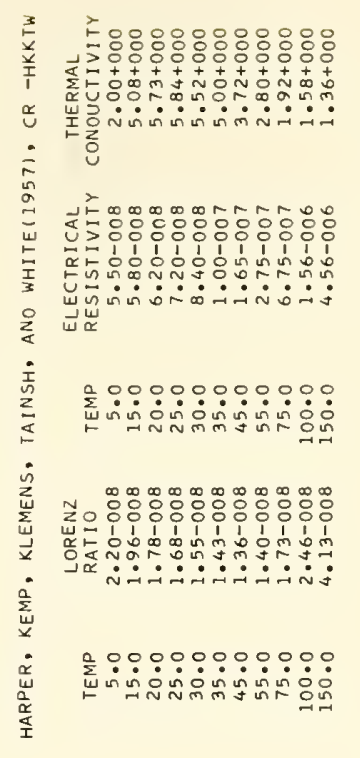

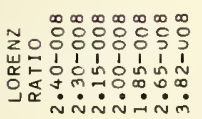

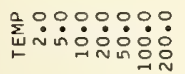

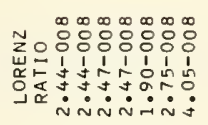

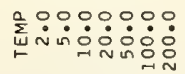

s.

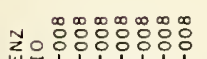

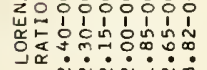

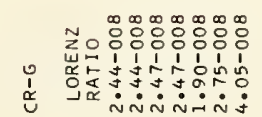

京

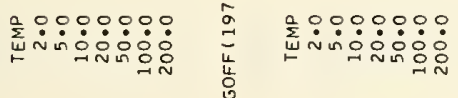

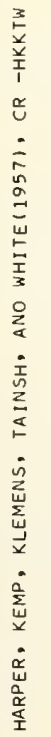

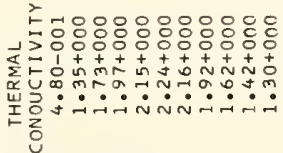

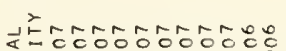

క\}

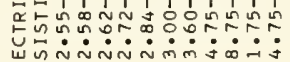
w岗

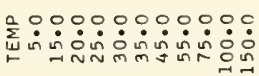

Nॅ :

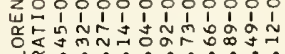

岌

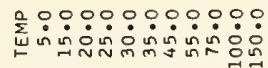

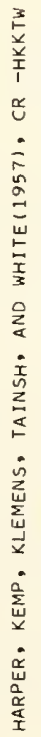

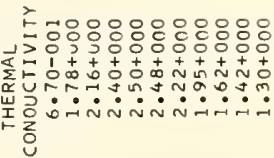

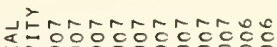

UZTiPOi

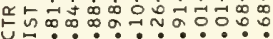

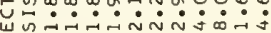
w

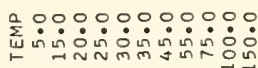

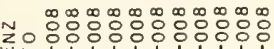

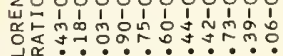

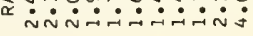

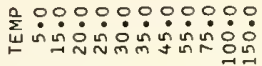




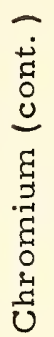
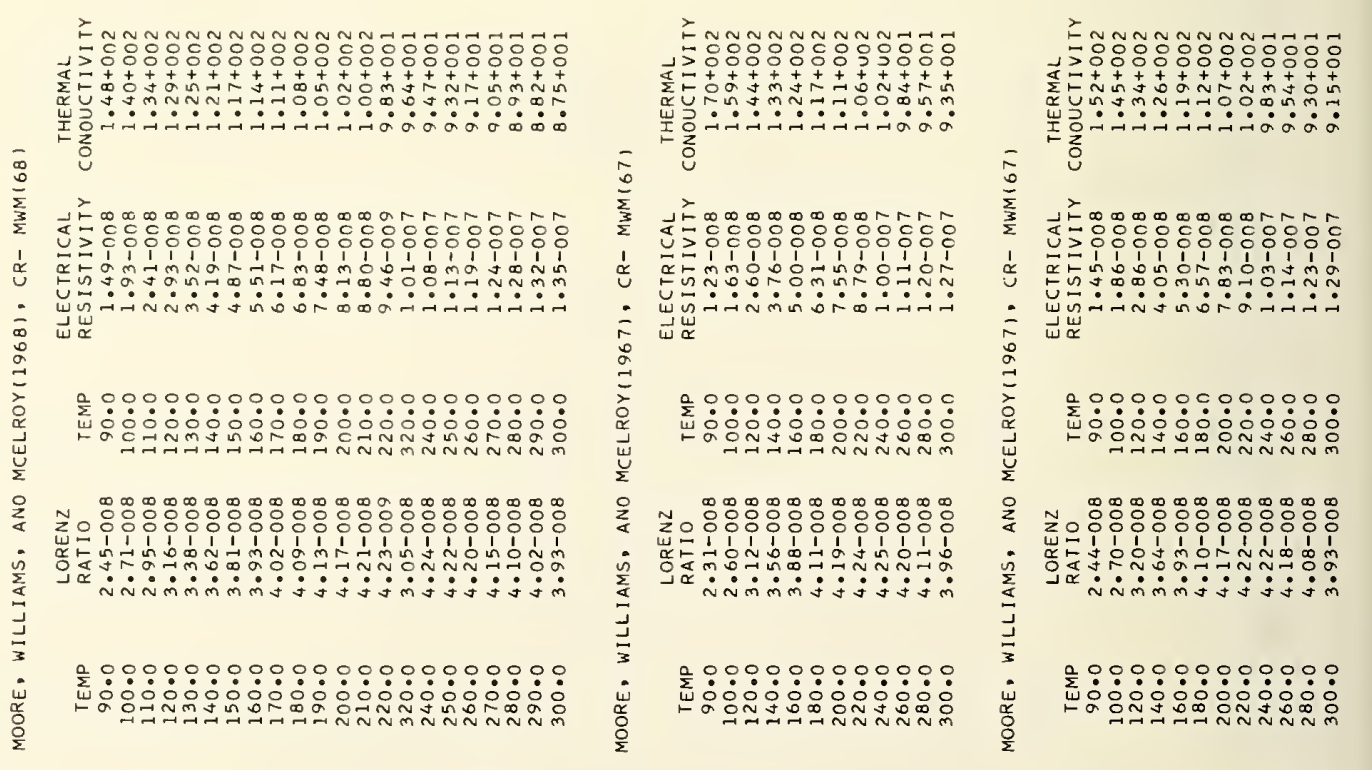


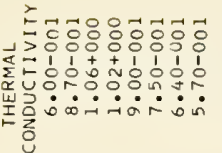

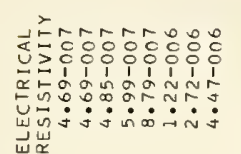

$\sum^{2}$

के

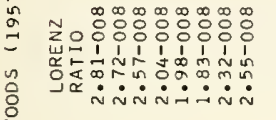

高

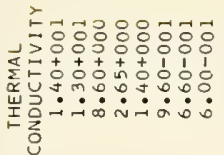

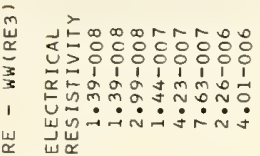

$\sum^{2}$

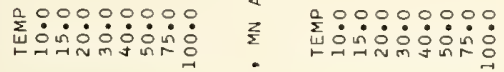

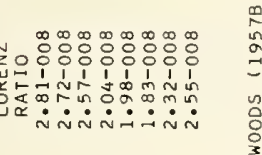

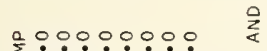

ㄱo:

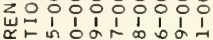

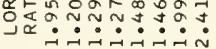

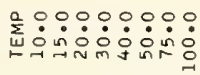

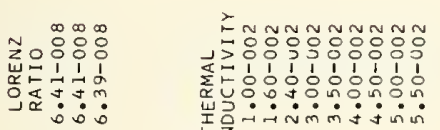

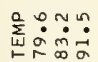

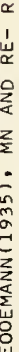

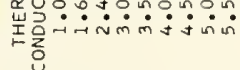

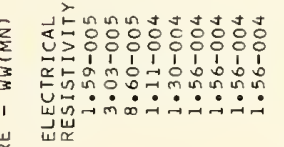

«

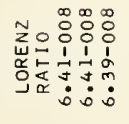

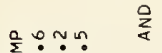

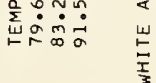

20:08:80:8:

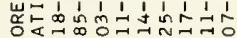

$0_{\infty}^{\infty}+\cdots$

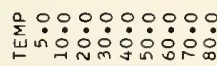

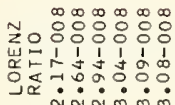

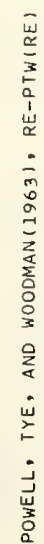

ㄱo

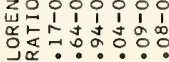

을

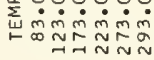

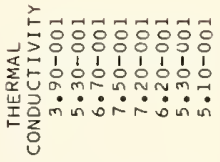

誌

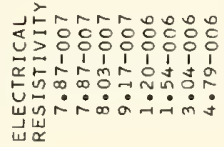



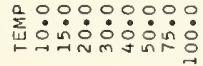

क

约

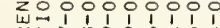

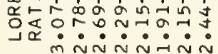

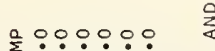

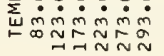

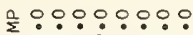

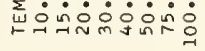




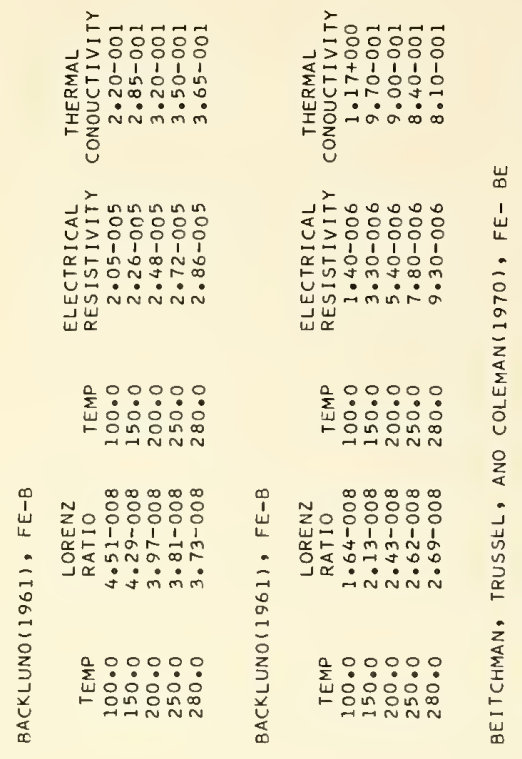

N

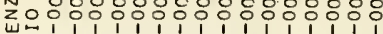

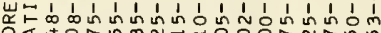

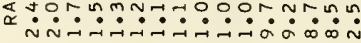

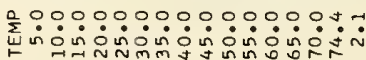

ㄴ.

1111111

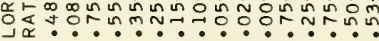

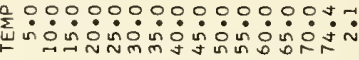

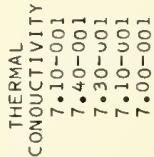

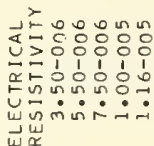

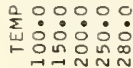

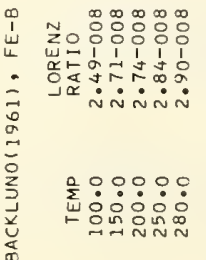

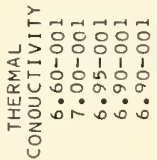

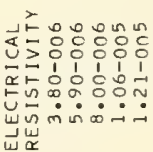

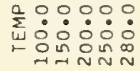

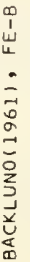

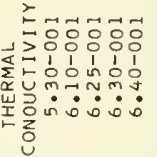

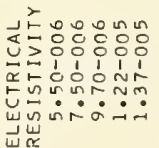

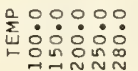

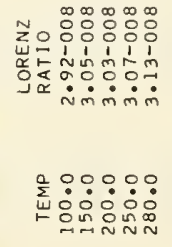

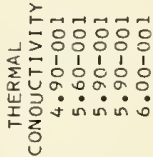

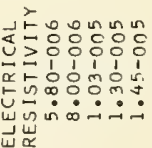

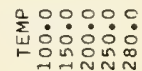

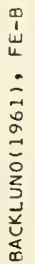

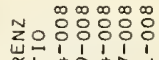

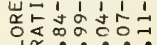
숳ำ

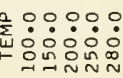
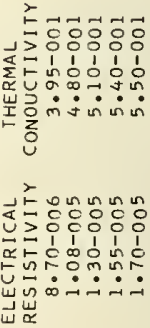

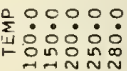

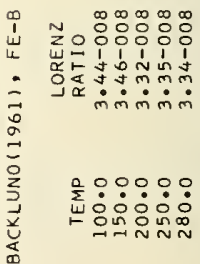




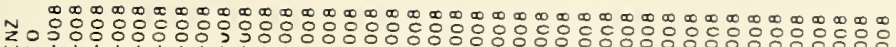

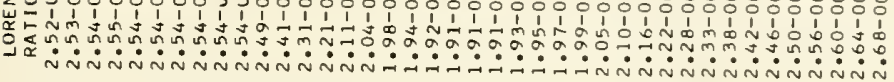

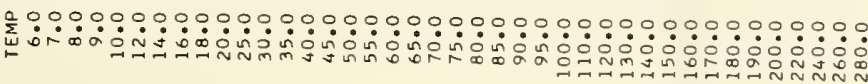

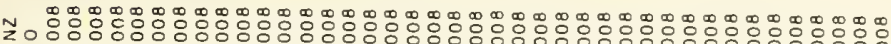

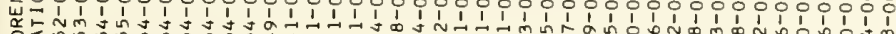

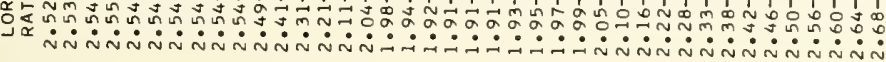

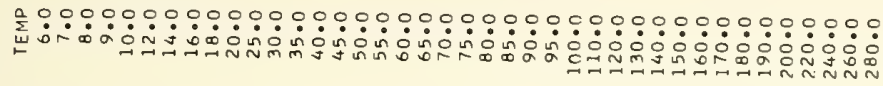

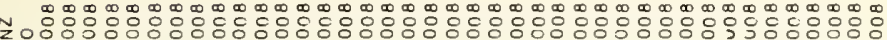

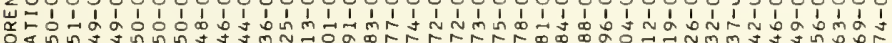

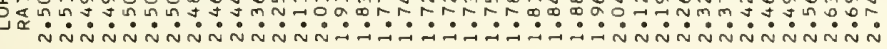

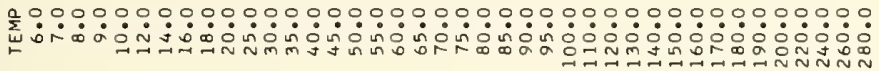

స̃.

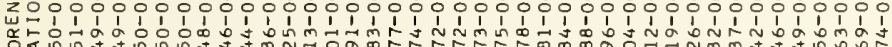

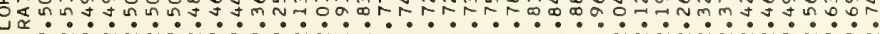

둔.

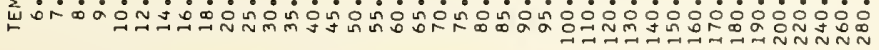




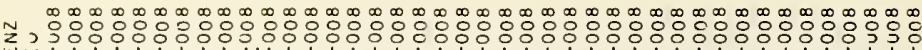

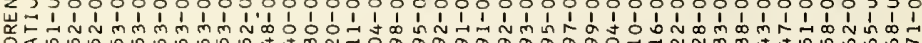

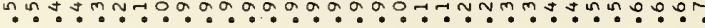

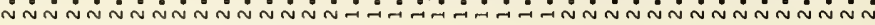

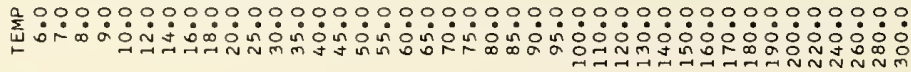

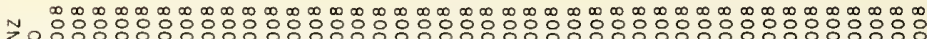
$z_{u} 0.0$

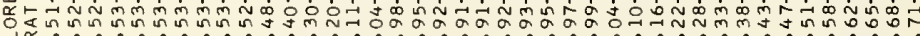

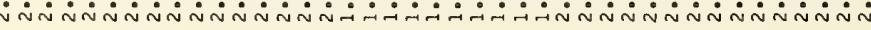

200000000000000000000000000000000000000000

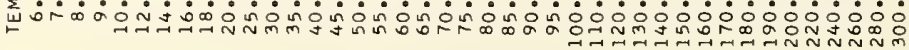

¿.

N

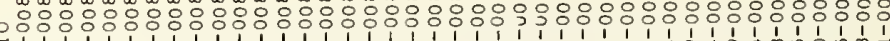

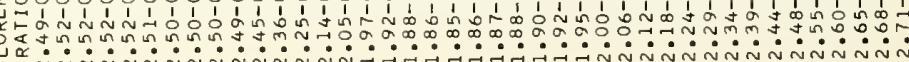
o.

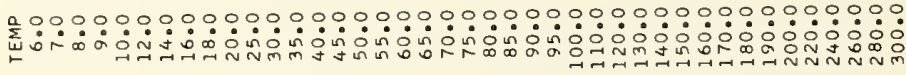

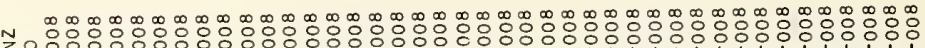

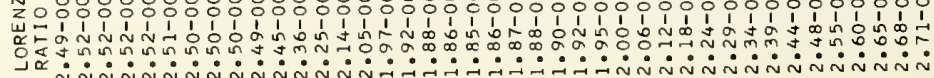

$00000000000000000000000000,00000,0,000.0000$

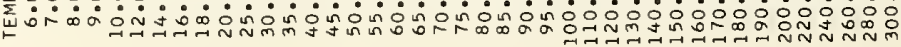




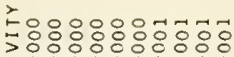

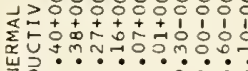

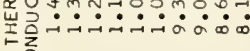

先:

$\because \geq 0$ 융ㅇㅇㅇㅛ

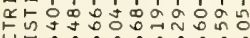

$\ddot{v}$

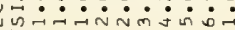

,

㟧

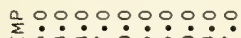

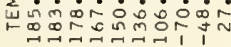

ㄱ.

zoOi i i i i i i

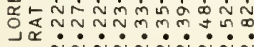

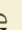

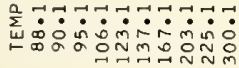
nNNNNNNNNN
N $\quad \infty \infty \infty \infty \infty \infty \infty \infty \infty \infty \infty \infty \infty$ ZOO

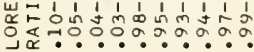

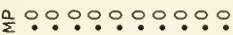

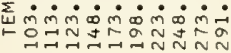

ㄱ. z

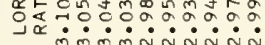

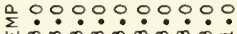
แำ

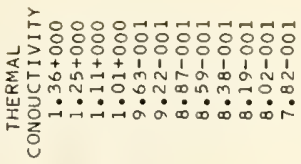

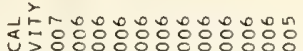

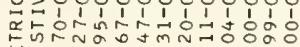

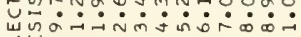
w

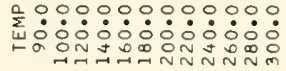

）

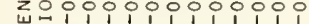

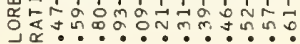

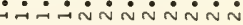

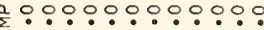

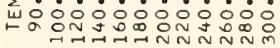

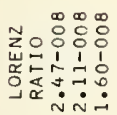

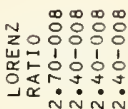

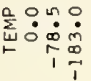

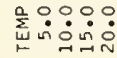

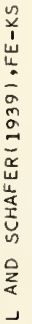

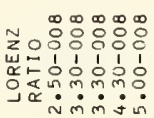

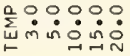

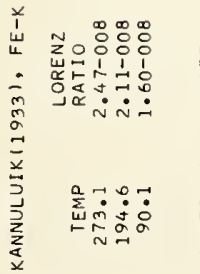

̌.

\section{ㄱo}

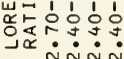

迹的品品

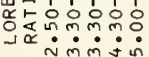

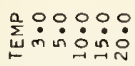

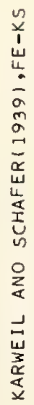

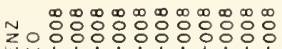

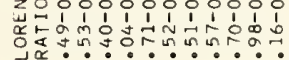

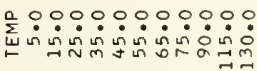

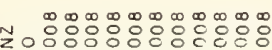

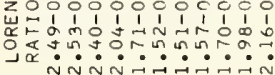

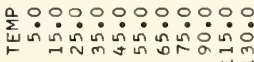

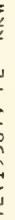

능ㅇㅇㅇㅇㅇㅇㅇㅇㅇㅇㅇㅇㅇㅇㅇ

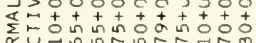

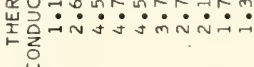

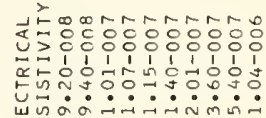
w山

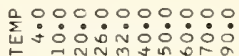

ㄱ. zo 11111111111

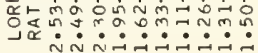

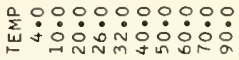




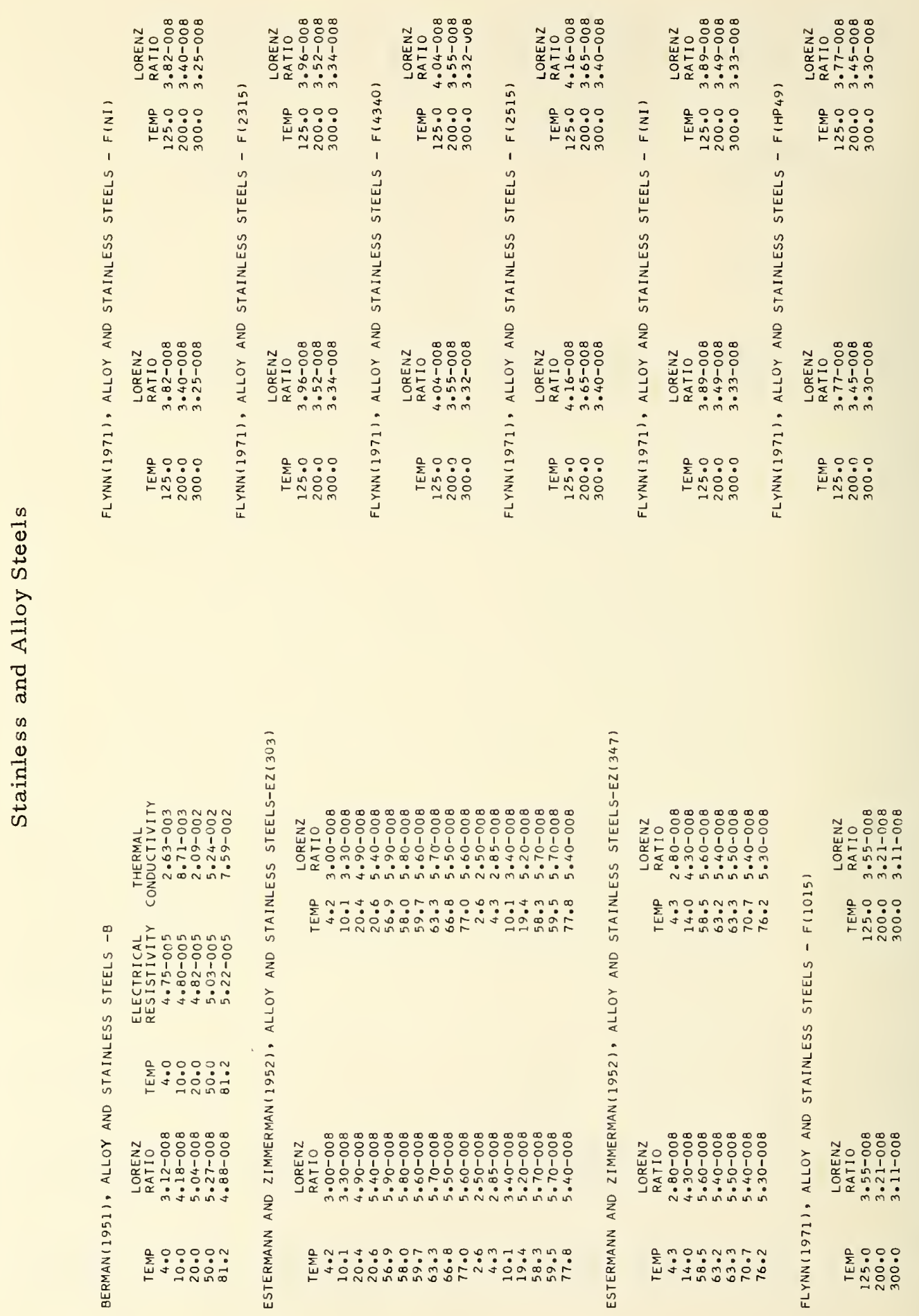


Nै

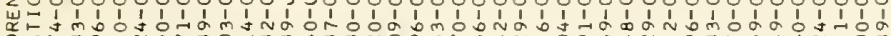

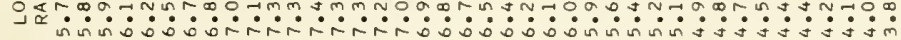

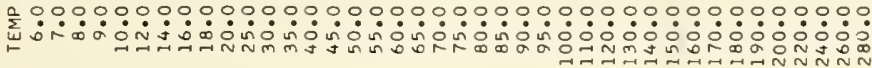

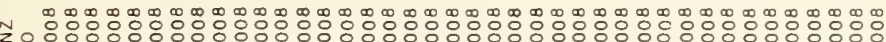

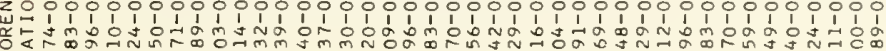

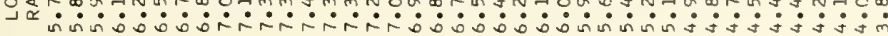

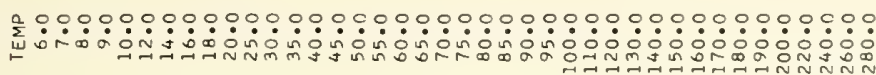

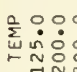
is
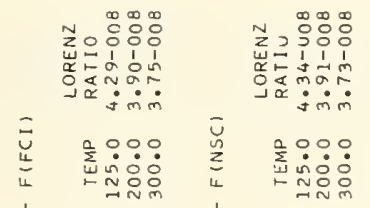

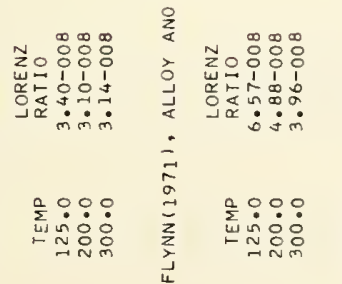


N U.

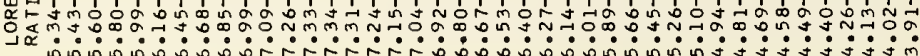

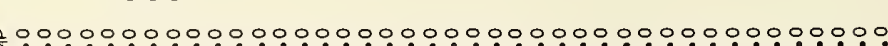

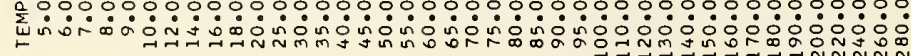

స̌

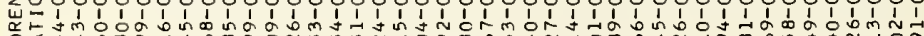

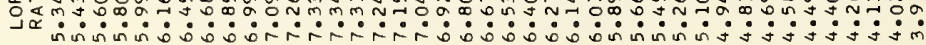

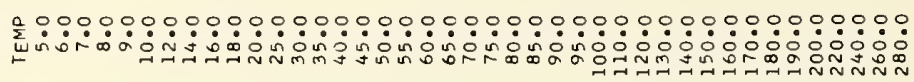

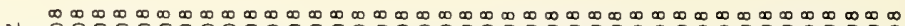

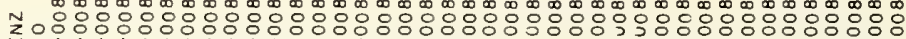

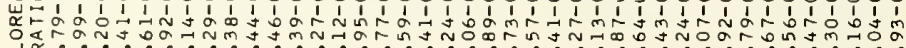

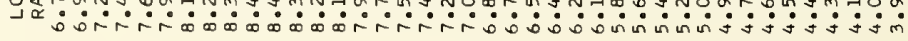

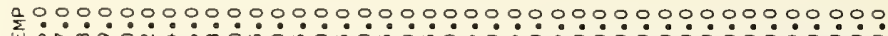

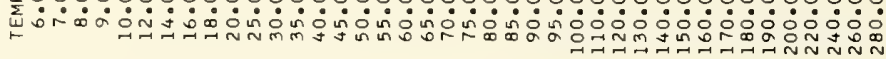

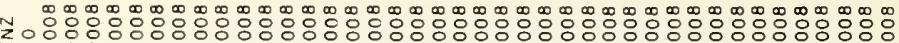

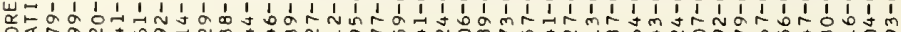

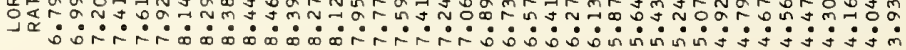

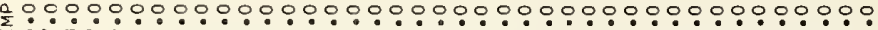

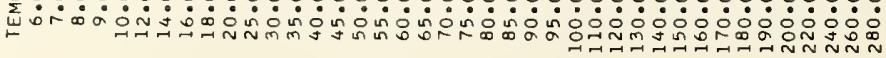


స

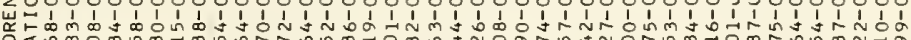

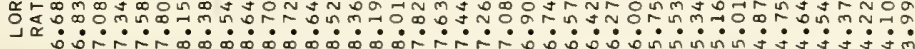

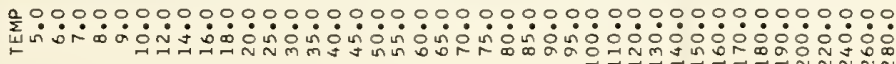

ح

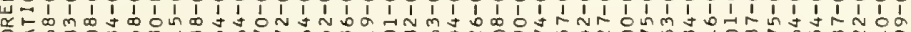

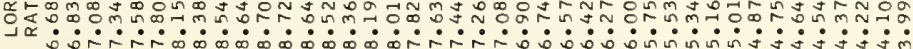

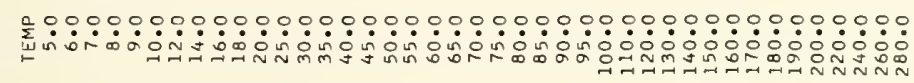

N

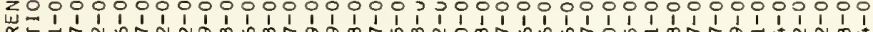

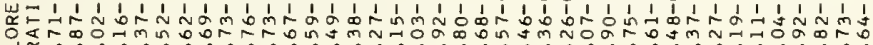

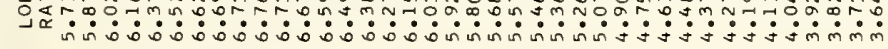

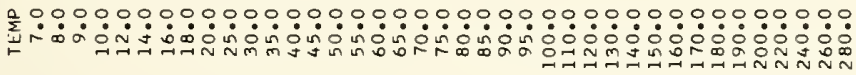

₹̌

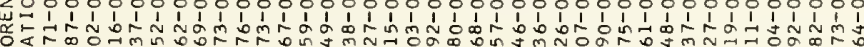

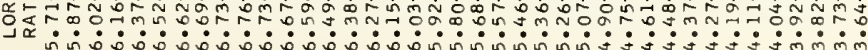




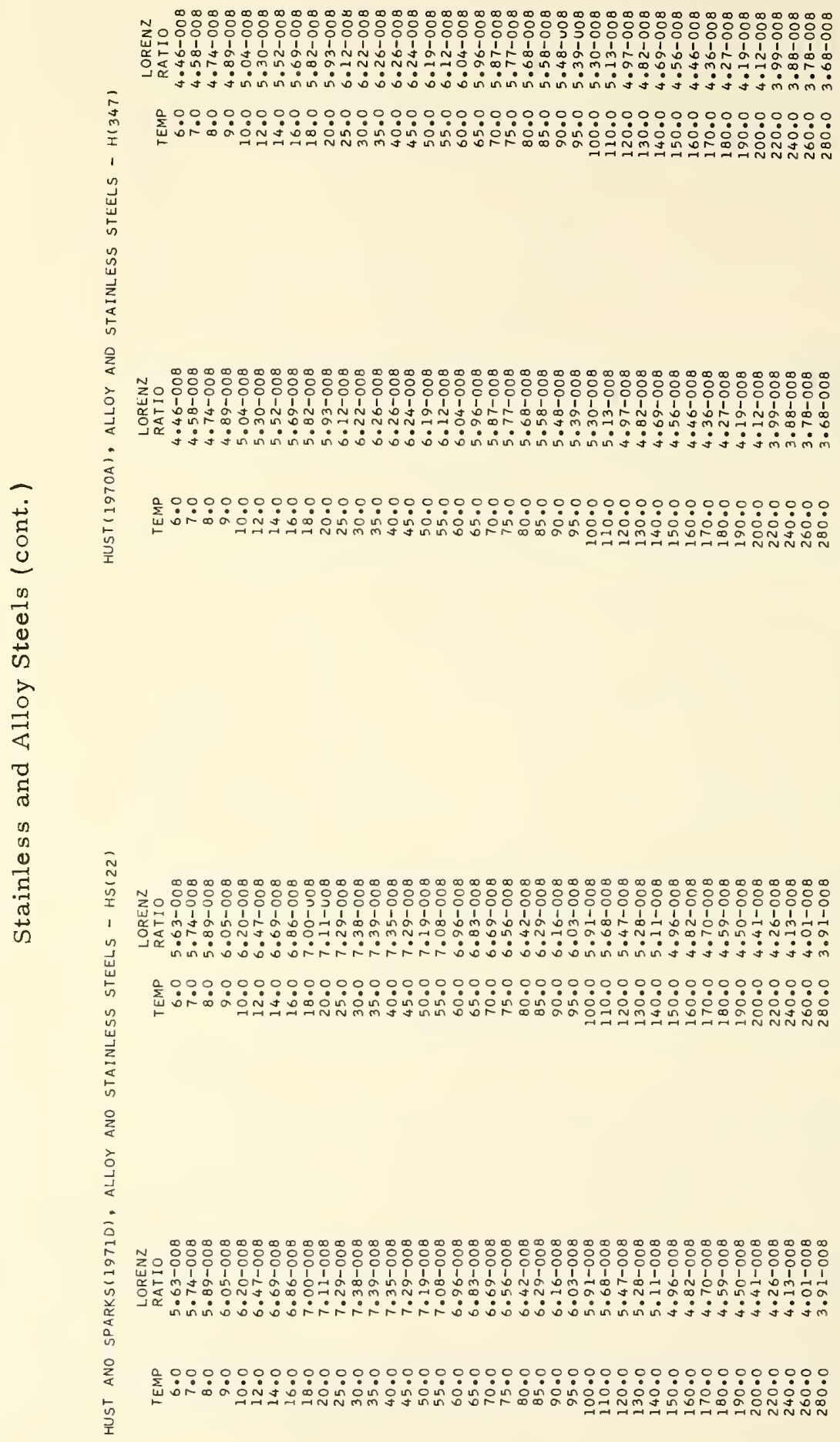



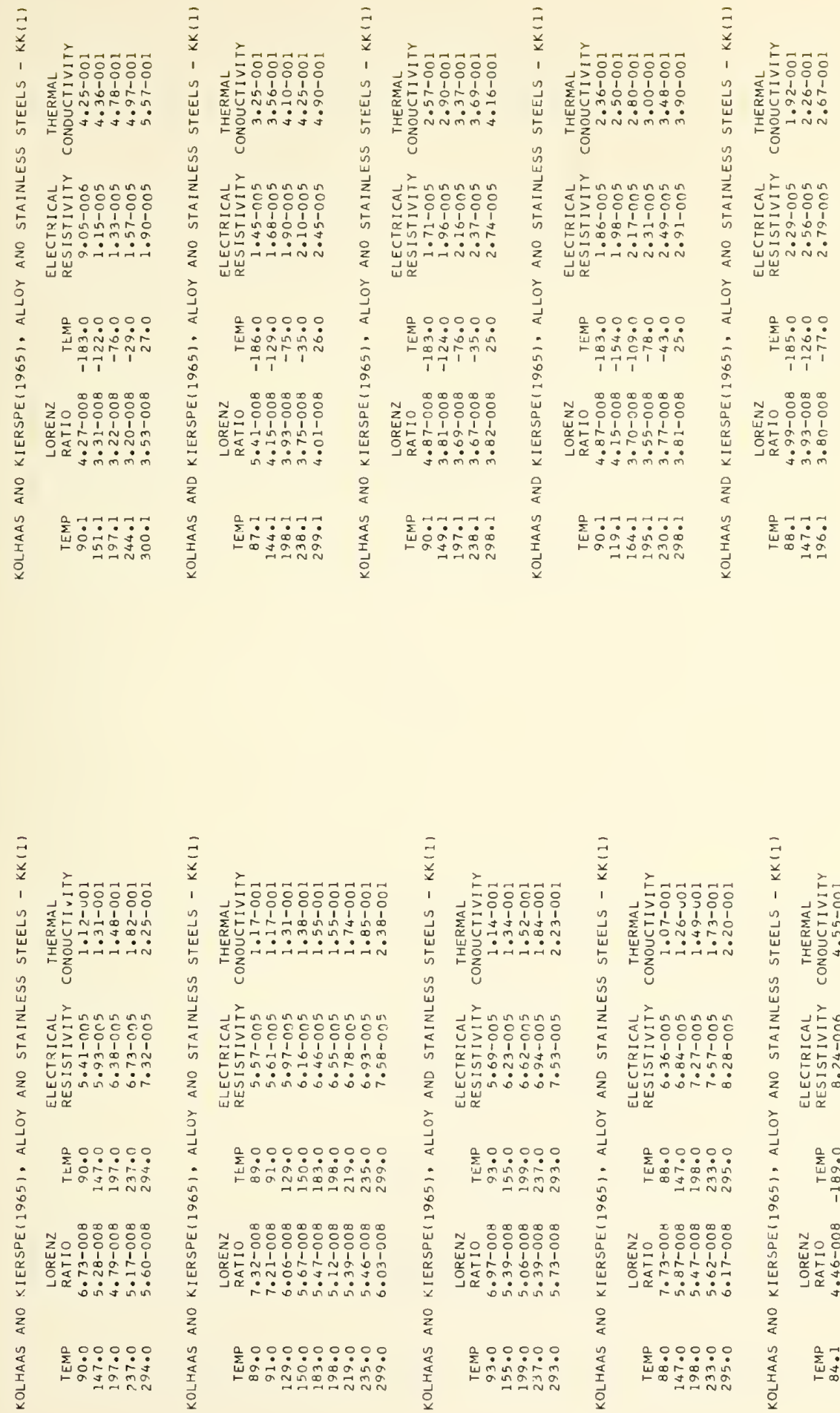

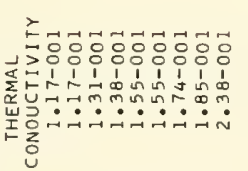

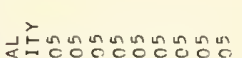

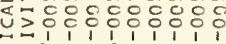

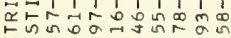

un $\dot{0} \dot{0} \dot{0} \dot{0} \dot{0} \dot{0}$ 近㟧

วิ

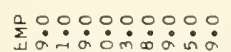

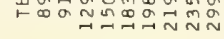
No:

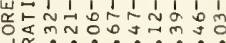

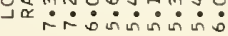

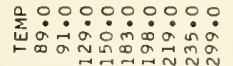

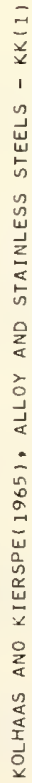

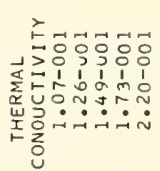

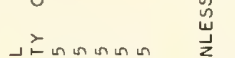

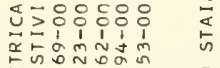

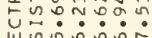

岃

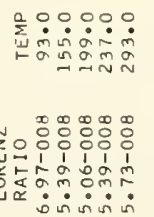

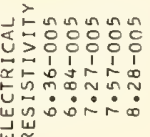
嵓㟧

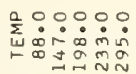

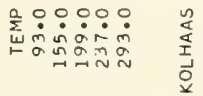

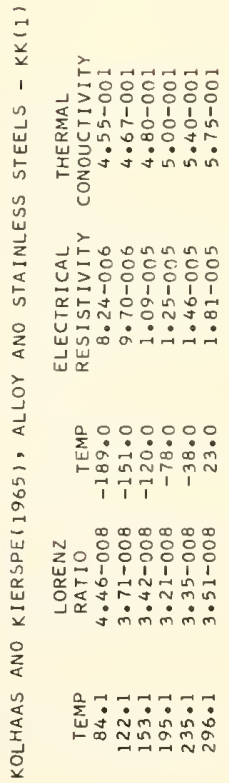




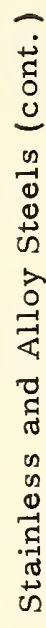

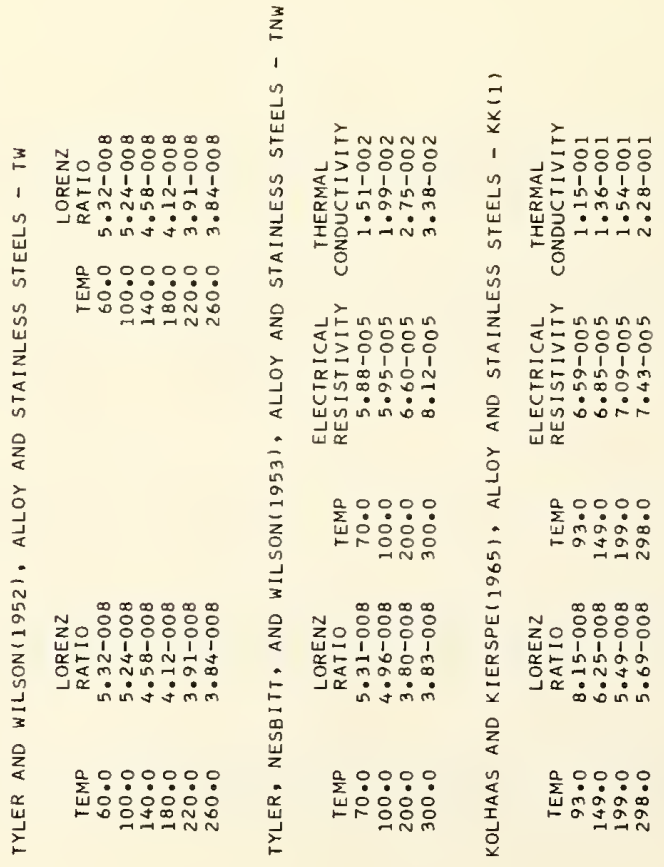




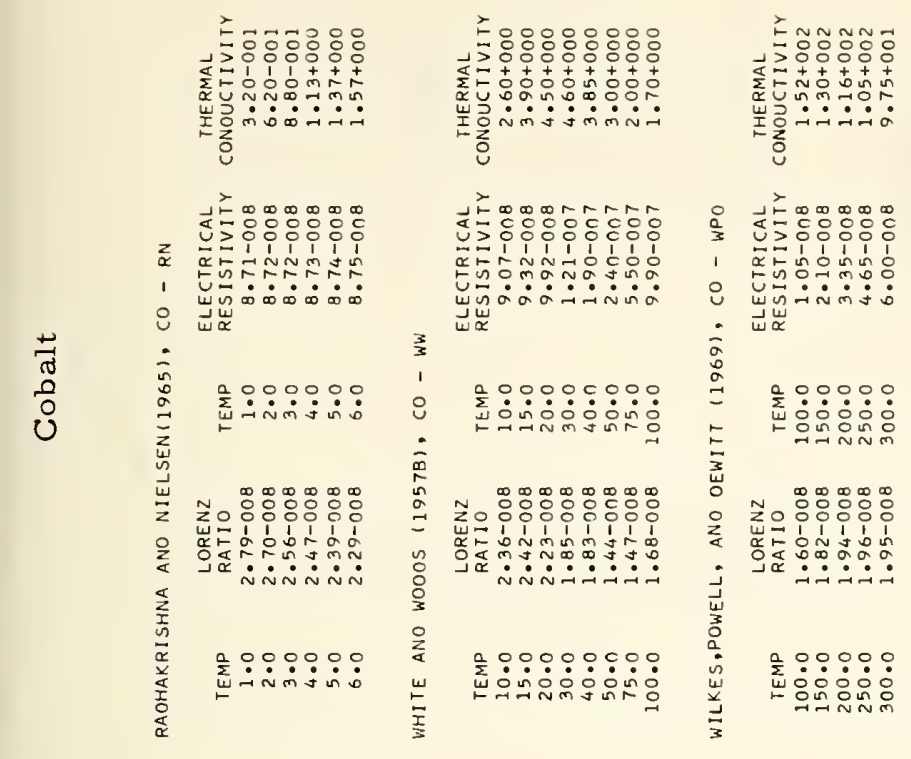

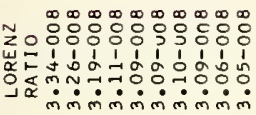

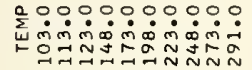

$n$
0
0
\pm
0
0
0
0
0
0

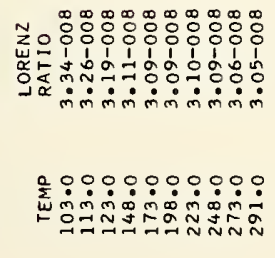




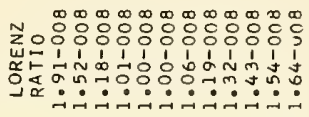

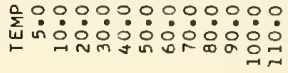

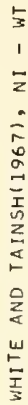

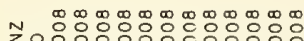

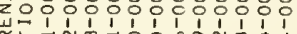

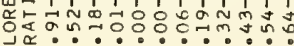

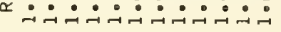

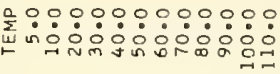

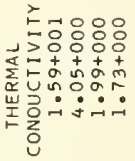

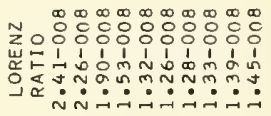

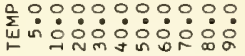

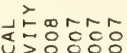

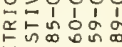

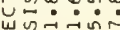

w $\underset{\sim}{\alpha}$

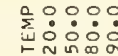

N

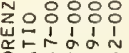

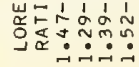

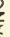

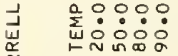

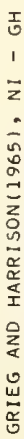

N N

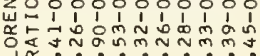

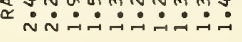

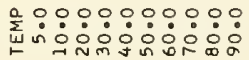

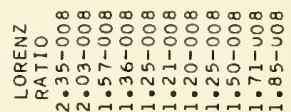

荘

$\underset{z}{\square}$

$\ddot{z}$

$\stackrel{\infty}{\infty}$

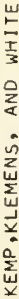

ㄴ.

Z00000000000

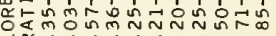

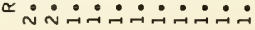

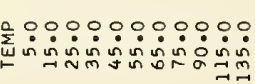

N స̌유요

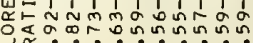

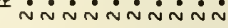

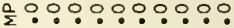

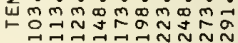

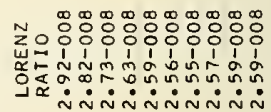

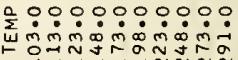




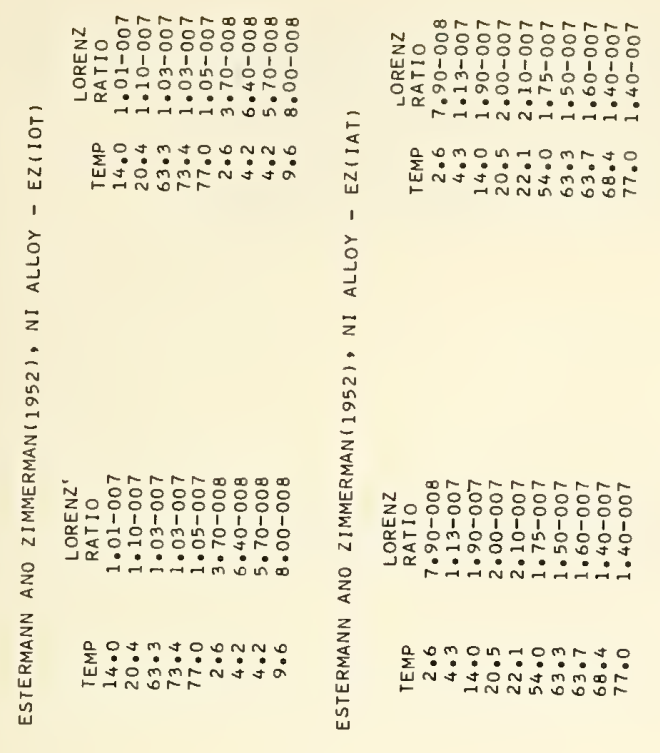

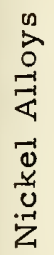

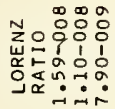

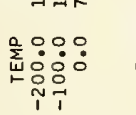

ำ

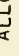

岁유잉

z

告
ㄴ.

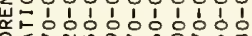
造:

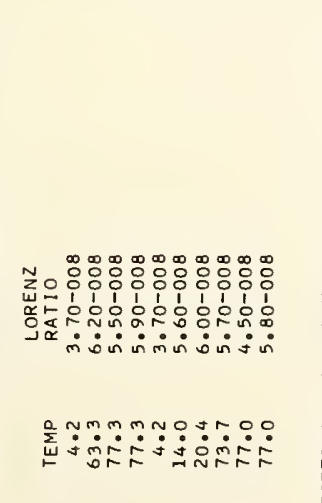

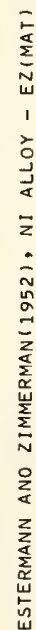

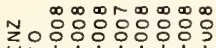

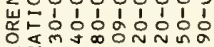

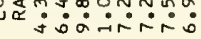

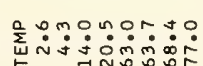

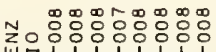

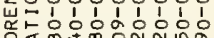

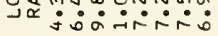

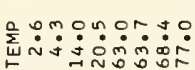

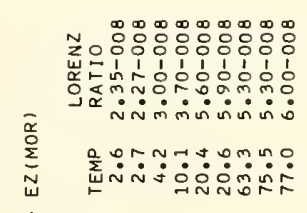

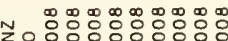

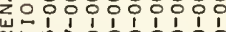

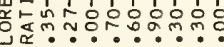
웅

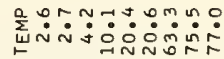




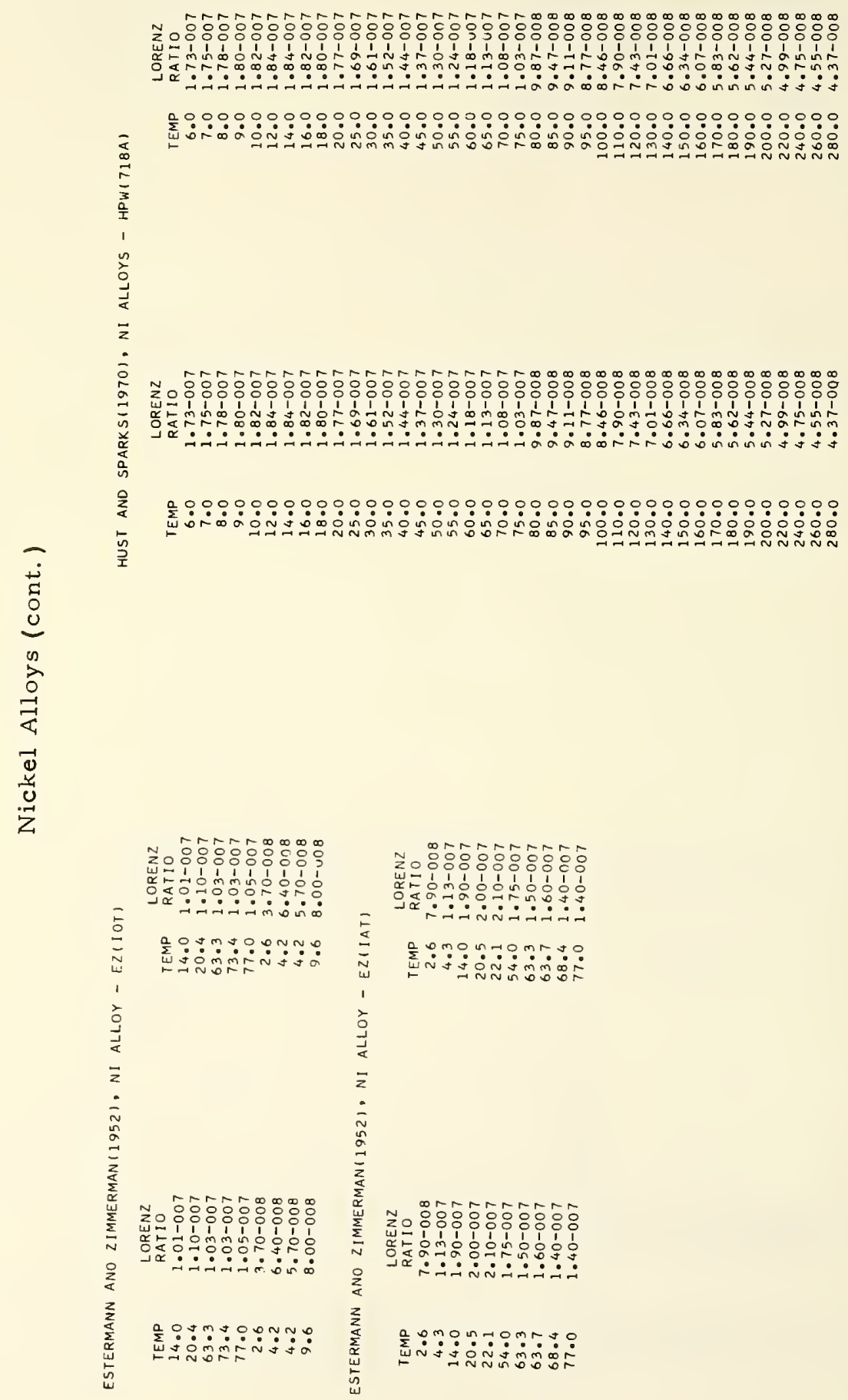

욱 


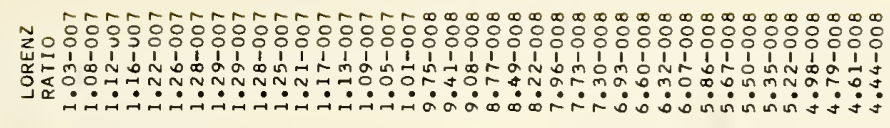

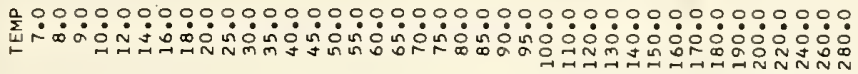

No

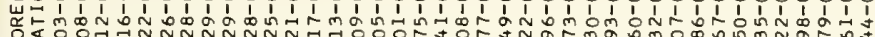

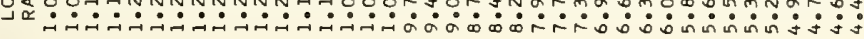

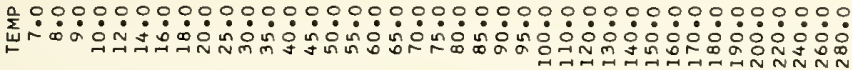

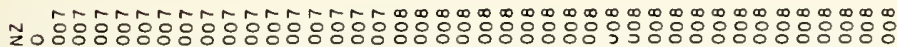

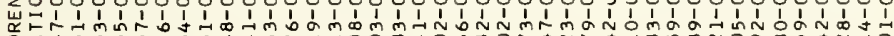

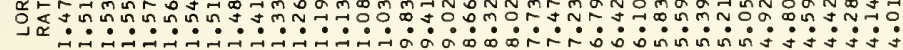

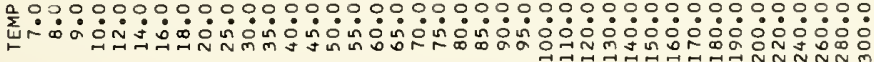

N

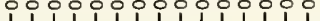

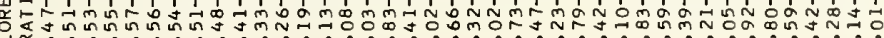

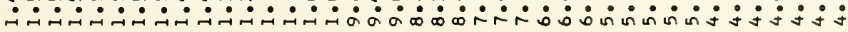

00000000000000000000000000000000000000000

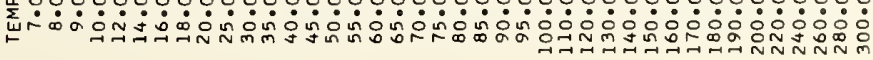



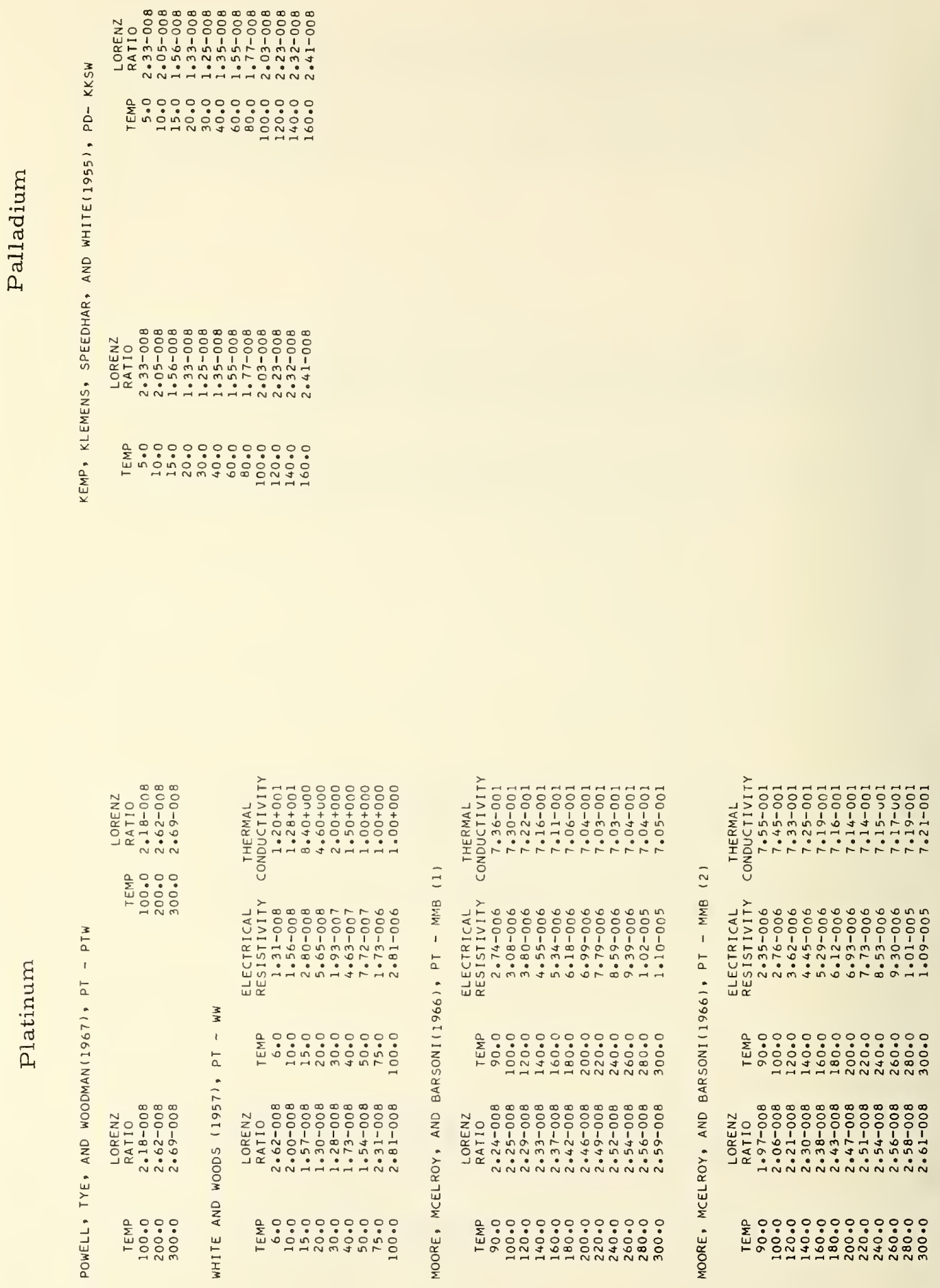


\section{Appendix III - Bibliography}

1. Alers, P. B., Spohr, D. A., and Webber, R. T., "The Thermal Conductivity of Metals", Conf. de Phys. des basses temps., Paris, 453-455 (Sept. 2-8, 1955).

2. Aliyev, N. G. and Volkenshteyn, N. V., "Low-Temperature Thermal Conductivity of Scandium and Yttrium", Fiz. Metal. Metalloved, 19, 793 (1965).

3. Allen, J. F., and Mendoza, E., "Thermal Conductivity of Copper and German Silver at Liquid Helium Temperature", Proc. Camb. Phil. Soc., 44, 280-288 (1947).

4. Amundsen, T., Myhre, A., and Salter, J. A. M., "The WiedemannFranz Ratio of Aluminum at Liquid Helium Temperatures", Phil. Mag., 25, 513-517 (1972).

5. Andrews, F. A., Webber, R. T., and Spohr, D. A., "Thermal Conductivities of Pure Metals at Low Temperatures I. Aluminum", 54, 994-996 (1951).

6. Aoyama, S., and Ito, T., "Thermal and Electrical Conductivities of Metals and Alloys at Low Temperature, Part I on Nickel-Copper Alloys", Nihon Kinzoku Gakkai-Shi (Journal of the Japan Institute of Metals), 4, 3-7 (1940).

7. Arajs, A., and Colvin, R. V., "Thermal Conductivity of Scandium Between 5 and $320^{\circ} \mathrm{K}$ ", 4th Conference on Rare Earth Research, 261-267 (1964).

8. Backlund, N. G., "An Experimental Investigation of the Electrical and Thermal Conductivity of Iron and Some Dilute Iron Alloys at Temperatures Above $100^{\circ} \mathrm{K} "$, J. Phys. Chem. Solids 20, 1-16 (1961).

9. Backlund, N. G., "Measurements and Analysis of the Thermal Conductivity of Tungsten and Molybdenum at 100-400 $\mathrm{K}^{\prime \prime}$, J. Phys. Chem. Solids 28, 2219-2223 (1967).

10. Beitchman, J. G., Trussel, C. W., and Coleman, R. V., "Electron Transport and Lorenz Number in Iron", Phys. Rev. Letters 25, $1291-1294$ (1970).

11. Berman, R., "The Thermal Conductivity of Some Alloys at Low Temperatures", Phil. Mag. 42, 642-650 (1951). 
12. de Haas, W. J., and de Nobel, J., "The Thermal and Electrical Resistance of a Tungsten Single Crystal at Low Temperatures and in Magnetic Fields", Physica 5, 449-463 (1938).

13. Estermann, I., and Zimmerman, J. E., "Heat Conduction in Alloys at Low Temperature", J. Appl. Phys. 23, 572-588 (1952).

14. Eucken, A., and Dittrich, K., "Zur Kenntnis des WiedemannFranzschen Gesetzes, II", Z. Physik, Chem. 129, 211-228 (1927).

15. Farrell, T., and Greig, D., "Thermal Conductivity of Nickel and Its Alloys", J. Phys. C (Solid State Phys.) 2, 1465-1473 (1969).

16. Fenton, E. W., Rogers, J. S., and Woods, S. B., "Lorenz Number of Pure Aluminum, Silver, and Gold at Low Temperatures", Can. J. Phys. 41, 2026-2033 (1963).

17. Flynn, D. R., Private Communication, (1971).

18. Goff, J. F., "Lorenz Number of Chromium", Phys. Rev. 1B, $1351-1362$ (1970).

19. Goens, E., and Grüneisen, E., "Electrical and Thermal Conductivity in Zinc and Cadmium Crystals", Ann. Physik 14, 164-180 (1932).

20. Grüneisen, E., and Goens, E., "Investigations on Metal Crystals. V. Electrical and Therrial Conductivity of Single and Poly-crystal Metals", Z. Physik 44, 615-642 (1927).

21. Harper, A.F. A., Kemp, W. R. G., Klemens, P. G., Tainsh, R. J., and White, G. K., "The Thermal and Electrical Conductivity of Chromium at Low Temperatures", Phil. Mag. 2, 577583 (1957).

22. Hust, J. G., "Thermal Conductivity Standard Reference Materials from 4 to $300 \mathrm{~K}$. I. Armco Iron", 9th Thermal Conductivity Conference, Ames Lab., USAEC; U.S. Office of Naval Research, Iowa State University (Conf. 691002), March 1970, p. 217-233, Oct 6-8 (1969).

23. Hust, J. G., "Thermal Conductivity Standard Reference Materials from 4 to 300 K. I. Armco Iron", unpublished NBS Report.

24. Hust J. G., "Thermal Conductivity, Electrical Resistiviey, and Thermopower of Aerospace Alloys from 4 to $300 \mathrm{~K}$. II. AISI 347 Stainless Steel", unpublished NBS Report. 
25. Hust, J. G., and Powell, R. L., "Thermal Conductivity of Aerospace Alloys at Crvogenic Temperatures", 8th Thermal Conductivity Conference, Oct 7-10 (1968), Purdue University, West Lafayette Indiana, Plenum Press, New York (1969).

26. Hust, J. G., Powell, R. L., and Weitzel, D. H., "Thermal Conductivity, Electrical Resistivity, and Thermopower of Aerospace Alloys from 4 to $300 \mathrm{~K}$ ", unpublished NBS Report.

27. Hust, J. G., Powell, R. L., and Weitzel, D. H., "Thermal Conductivity Standard Reference Materials from 4 to $300 \mathrm{~K}$. I. Armco Iron: Including Apparatus Description and Error Analysis", J. Res. Nat. Bur. Stand. (U.S.), 74A, 673-690 (1970).

28. Hust, J. G., and Sparks, L. L., "Thermal Conductivity, Electrical Resistivity, and Thermopower of Aerospace Alloys from 4 to $300 \mathrm{~K}$. III. Annealed Inconel 718", unpublished NBS Report.

29. Hust, J. G., and Sparks, L. L., "Thermal Conductivity Standard Reference Materials from 4 to $300 \mathrm{~K}$. II. OSRM Iron 1265", unpublished NBS Report.

30. Hust, J. G., and Sparks, L. L., "Thermal Conductivity Standard Reference Materials from 4 to $300 \mathrm{~K}$ : IV. AGARD Gold", unpublished NBS Report.

31. Hust, J. G., and Sparks, L. L., "Thermal Conductivity Standard Reference Materials from 4 to $300 \mathrm{~K}$. III. AGARD Stainless Steel", unpublished NBS Report.

32. Hust, J. G., and Sparks, L. L., "Thermal Conductivity, Electrical Resistivity, and Thermopower of Aerospace Alloys from 4 to $300 \mathrm{~K}$ : IV. A286 Stainless Steel", unpublished NBS Report.

33. Hust, J. G., and Sparks, L. L., "Thermal Conductivity, Electrical Resistivity, and Thermopower of Aerospace Alloys from 4 to 300 K: V. Aluminum 2024", unpublished NBS Report.

34. Hust, J. G., and Sparks, L. L., "Thermal Conductivity, Electrical Resistivity, and Thermopower of Aerospace Alloys from 4 to 300 $\mathrm{K}$ : VI. $\mathrm{Fe}-22 \mathrm{Cr}-13 \mathrm{Ni}-5 \mathrm{Mr}$ Stainless Steel", unpublished NBS Report.

35. Hust, J. G., and Sparks, L. L., "Thermal Conductivity Standard Reference Materials from 4 to $300 \mathrm{~K}$ : V. AGARD Stainless Steel", unpublished NBS Report. 
36. Hust, J. G., and Sparks, L. L., "Standard Reference Materials: Thermal Conductivity of Electrolytic Iron, SRM 734, from 4 to 300 K"', Nat. Bur. Stand. (U.S.), Spec. Publ. 260-31 (1971f).

37. Hust, J. G., and Sparks, L. L., "Standard Reference Materials: Thermal Conductivity of Austenitic Stainless Steel, SRM 735, from 5 to $280 \mathrm{~K}^{\prime \prime}$, Nat. Bur. Stand. (U.S.), Spec. Publ. 260-35 (1972).

38. Hust, J. G., Weitzel, D. H., and Powell, R. L., "Thermal Conductivity of Aerospace Alloys at Cryogenic Temperatures", 7 th Thermal Conductivity Conference, Nov. 13-16, 1967, Gaithersburg, Md. (Nat. Bur. Stand., Spec. Publ. 302, pp. 271 278 (1968).

39. Hust, J. G., Weitzel, D. H., and Powell, R. L., "Thermal Conductivity, Electrical Resistivity, and Thermopower of Aerospace Alloys from 4 to $300 \mathrm{~K}^{\prime \prime}$, J. Res. Nat. Bur. Stand. (U.S.), 75A, $269-277$ ( 1971 ).

40. Kannuluik, W. G., "On the Thermal Conductivity of Some Metal Wires", Proc. Roy. Soc. A131, 320-335 (1931).

41. Kannuluik, W. G., "The Thermal and Electrical Conductivities of Several Metals Between $183^{\circ} \mathrm{C}$ and $100^{\circ} \mathrm{C}$, Proc. Roy. Soc. A 141, 159-168 (1933).

42. Karweil, J., and Schafer, K., "The Thermal Conductivity of Poorly Conducting Alloys Between 3 and $20^{\circ} \mathrm{K} "$, Ann. Physik 36, $567-577$ (1939).

43. Kemp, W. R. G., Klemens, P. G., Sreedhar, A. K., and White, G. K., "The Thermal and Electrical Conductivity of Palladium at Low Temperatures", Phil. Mag. 46, $811-814$ (1955).

44. Kemp, W. R. G., Klemens, P. G., and Tainsh, R. J., "Lattice Thermal Conductivity of Some Copper Alloys", Aust. J. Phys. 10, 454-461 (1957).

45. Kemp, W. R. G., Klemens, P. G., Tainsh, R. J., "Thermal and Electrical Conductivities of Rhodium and Iron", Ann. Phys. (Ger.) 5, 35-41 (1959).

46. Kemp, W. R. G., Klemens, P. G., Tainsh, R. J., "The Lattice Thermal Conductivity of Copper Alloys: Effect of Plastic Deformation and Annealing", Phil. Mag. 4, 845-857 (1959b). 
47. Kemp, W. R. G., Klemens, P. G., White, G. K., "Thermal and Electrical Conductivities of Iron, Nickel, Titanium, and Zirconium at Low Temperatures", Aust. J. Phys. 9, 180-188 (1956).

48. Kohlhaas, R., and Kierspe, W., "Thermal Conductivity of Pure Iron and Some Ferritic and Austenitic Steels Between the Temperature of Liquid Air and Room Temperature", Arch. Eisenhüttenw 36, 301-309 (1965).

49. Lees, C. H., "XI Bakerian Lecture - The Effects of Temperature and Pressure on the Thermal Conductivities of Solids", Phil. Trans. Roy. Soc. A208, $381-443$ (1908).

50. Lewis, E. J., "Some Thermal and Electrical Properties of Berythium", Phys. Rev. 34, 157-187 (1929).

51. Malm, H. L., and Woods, S. B., "The Low-Temperature Thermal Conductivity Electrical Resistivity, and Thermoelectric Power of Dilute Silver Alloys with Manganese", Can. J. Phys. 44, 2293-2309 (1966).

52. Mannchen, W., "Thermal, Electrical Conductivities, and Lorenz Number of Light Metal Alloys", Z. Metal Kunde 23, 193-196 (1931).

53. Moore, J. P., McElroy, D. L., Barisoni, M., "Thermal Conductivity Measurements Between 78 and $340^{\circ} \mathrm{K}$ on Aluminum, Iron, Platinum, and Tungsten", 6th Thermal Conductivity Conference, Oct. 19-21 1966, Air Force Materials Laboratory, Dayton, Ohio, pp. 737-778

54. Moore, J. P., McElroy, D. L., and Graves, R. S., "Thermal Conductivity and Electrical Resistivity of High-Purity Copper from 78 to $400^{\circ} \mathrm{K}^{\prime \prime}$, Can. J. Phys. 45, 3849-3865 (1967).

55. Moore, J. P., Williams, R. K., and McElroy, D. L., "Physical Properties of Chromium from 77 to $400^{\circ} \mathrm{K}^{\prime \prime}$, 7th Thermal Conductivity Conference, Nov. 13-16 (1967), National Bureau of Standards, Gaithersburg, Md., NBS, Spec. Publ. 302, pp. 297-310 (1968).

56. Moore, J. P., Williams, R. K., and McElroy, D. L., "Further Comments on Transport Properties of Chromium", 8th Thermal Conductivity Conference, Oct. 7-10 (1968), Purdue University, West Lafayette, Indiana, Plenum Press, New York, pp. 303 313 (1969).

57. Powel1, R. L., Bunch, M. D., and Gibson, E. F., "Low-Temperature Transport Properties of Commercial Metals and Alloys", J. Appl. Phys. 31, 504-505 (1960). 
58. Powell, R. L., Hall, W. J., and Roder, H. M., "Low-Temperature Transport Properties of Commercial Metals and Alloys", J. Appl. Phys. 31, 496-503 (1960).

59. Powell, R. L., Harden, J. L., and Gibson, E. F., "Low Temperature Transport Properties of Commercial Metals and Alloys", J. Appl. Phys. 31, 1221-1224 (1961).

60. Powell, R. L., Roder, H. M., and Hall, W. J., "Low Temperature Transport Properties of Copper and Its Dilute Alloys Pure Copper Annealed and Cold-Drawn", Phys. Rev. 115, 314-323 (1959).

61. Powell, R. W., and Jolliffe, B. W., "The Thermal Conductivities of Scandium and Some Rare Earth Metals", Phys. Letters 14, $171-172$ (1965).

62. Powell, R. W., and Tye, R. P., "The Thermal and Electrical Conductivity of Titanium and Its Alloys", J. Less-Common Metals 3, $226-233$ (1961).

63. Powell, R. W., Tye, R. P., and Woodman, M. J., "Thermal Conductivities and Electrical Resistivities of the Platinum Metals", Platinum Metals Rev. 6, $138-143$ (1962).

64. Powell, R. W., Tye, R. P., and Woodman, M. J., "The Thermal Conductivity and Electrical Resistivity of Polycrystalline Metals of the Platinum Group and of Single Crystals of Ruthenium", J. Less-Common Metals 12, 1-10 (1967).

65. Powell, R. W., Tye, R. P., and Woodman, M. J., "The Thermal Conductivity of Pure and Alloyed Aluminum I. Solid Aluminum as a Reference Material", Adv. in Thermophysical Properties at Extreme Temperatures and Pressures, Proc. 3rd Symp., Purdue, ASME (1965) pp. 277-288.

66. Radhakrishna, P., and Nielsen, M., "Transport Properties of Cobalt at Low Temperature", Phys. Stat. Sol. 11, 111-115 (1965).

67. Reddemann, H., "Wiedemann - Franz Number of $\beta$ - Manganese at $-190^{\circ} \mathrm{C}^{\prime \prime}$, Ann. Physik 22, 28-30 (1935).

68. Staebler, J., "Electrical and Thermal Conductivities and Wiedemann Franz Number of Light Metals and Magnesium Alloys", Doctoral Thesis Submitted to Techischen Hochshule, Beslau (1929).

69. Tamarin, P. V., Chuprikov, G. E., and Shalyt, S. S., "Investigation of the Phenomena of Charge and Heat Transfer in Yttrium Single Crystals at Low Temperatures", Sov. Phys. J.E.T.P. 28, 836-837 (1969). 
70. Tyler, W. W., Nesbitt, L. B., and Wilson, Jr., A. C., "Some Low Temperature Properties of Titanium Alloy RC-130-B and Stainless Stee1", J. Metals, 1104-1105 (1953).

71. Tyler, W. W., and Wilson, Jr., A. C., "Thermal Conductivity, Electrical Resistivity, and Thermoelectric Power of Titanium Alloy RC-130-B", General Electrical Co., Knolls Atomic Power Laboratory, Report No. KAPL - 803 (1952) Contr. No. W - 31 - 109 Eng 52, 41 .

72. Wagner, D. K., Garland, J. C., and Bowers, R., "Low-Temperature Electrical and Thermal Resistivities of Tungsten", Phys. Rev. 3B, $3141-3149$ (1971).

73. White, G. K., "The Thermal and Electrical Conductivity of Copper at Low Temperature", Aust. J. Phys. 6, 397-404 (1953).

74. White, G. K., and Tainsh, R. J., "Electron Scattering in Nickel at Low Temperatures", Phys. Rev. Letters 165 (1967).

75. White, G. K., and Tainsh, R. J., "Lorenz Number for High-Purity Copper", Phys. Rev. 119, 1869-1871 (1960).

76. White, G. K., and Woods, S. B., "Thermal and Electrical Conductivities of Solids at Low Temperatures", Can. J. Phys. 33, 58-73 (1955).

77. White, G. K., and Woods, S. B., "Thermal and Electrical Conductivity of Rhodium, Iridium, and Platinum", Can. J. Phys. 35, 248257 (1957).

78. White, G. K., and Woods, S. B., "Low Temperature Resistivity of Transition Elements: Vanadium, Niobium, and Hafnium", Can. J. Phys. 35, 892-900 (1957a).

79. White, G. K., and Woods, S. B., "Low Temperature Resistivity of the Transition Elements: Cobalt, Tungsten, and Rhenium", Can. J. Phys. 35, 656-665 (1957).

80. White, G. K., and Woods, S. B., "Conductivity of $\alpha$ - Manganese", Can. J. Phys. 35, 346-348 (1957c).

81. Wilkes, K. E., and Powell, R. W., "Thermal Conductivity of Aluminum Between about 78 and $373 \mathrm{~K} "$ ", 7 th Thermal Conductivity Conference, NBS, Gaithersburg, Md. Nov. 13-16, 1967, Nat. Bur. Stand. (U.S.) Spec. Publ. 302, 293-296 (1968). 
82. Wilkes, K. E., Powell, R. W., and Dewitt, D. P., "Thermal Conductivity Measurements on Cobalt and Zinc Between 78 and 373K", 8th Thermal Conductivity Conference, Oct. 7-10, 1968, Purdue University, West Lafayette, Indiana, Plenum Press, New York, pp. 3-8 (1969). 
1. PUBLICATION OR REPORT NO.

NBS TN-634

4. TITLE AND SUBTITLE

Lorenz Ratios of Technically Important Metals and Alloys Gor't Accession
No.

3. Recipient's Accession No.

5. Publication Date

February 1973

6. Performing Organization Code

8. Performing Organization

7. AUTHOR(S)

J. G. Hust and L. L. Sparks

9. PERF ORMING ORGANIZATION NAME AND ADDRESS

NATIONAL BUREAU OF STANDARDS, Boulder Labs DEP ARTMENT OF COMMERCE

WASHINGTON, D.C. 20234

12. Sponsoring Organization Name and Address

13. Type of Report \& Period Covered

Same as Item 9.

14. Sponsoring Agency Code

16. ABSTRACT (A 200-word or less factual summaty of most significant information. If document includes a significant bibliography or literature survey, mention it here.)

A comprehensive review and compilation of the world literature on Lorenz ratio of technically important metals and alloys is presented. Lorenz ratio, electrical resistivity, thermal conductivity and characterization data are compiled in tabular form and the Lorenz ratio data are presented in graphical form as well. Data are included here only if the research reported both thermal conductivity and electrical resistivity of the specimens. No attempt has been made to smooth data or present recommended values.

17. KEY WORDS (Alphabetical order, separated by semicolons)

Alloys; compilation; cryogenic; electrical resistivity; Lorenz Ratio; metals; thermal conductivity.

18. AVAILABILITY STATEMENT

$\mathrm{X}$ UNL IMITED.

FOR OFFICIAL DISTRIBUTION. DO NOT RELEASE TO NTIS.
19. SECURITY CLASS (THIS REPORT)

UNCL ASSIF IED

20. SECURITY CLASS (THIS PAGE)
21. NO. OF PAGES

$$
133
$$

22. Price

$\$ 1.25$ 



\section{PERIODICALS}

JOURNAL OF RESEARCH reports National Bureau of Standards research and development in phrsics, mathematics, and chemistry. Comprehensive scientific papers give complete details of the work, including laboratory data, experimental procedures, and theoretical and mathematical analyses. Illustrated with photographs, drawings, and charts. Includes listings of other NBS papers as issued.

Published in two sections, available separately:

\section{- Physics and Chemistry (Section A)}

Papers of interest primarily to scientists working in these fields. This section covers a broad range of physical and chemical research, with major emphasis on standards of physical measurement, fundamental constants, and properties of matter. Issued six times a year. Annual subscription: Domestic, $\$ 17.00$; Foreign, \$21.25.

\section{- Mathematical Sciences (Section B)}

Studies and compilations designed mainly for the mathematician and theoretical physicist. Topics in mathematical statistics, theory of experiment design, numerical analysis, theoretical physics and chemistry, logical design and programming of computers and computer systems. Short numerical tables. Issued quarterly. Annual subscription: Domestic, $\$ 9.00$; Foreign, $\$ 11.25$.

\section{TECHNICAL NEWS BULLETIN}

The best single source of information concerning the Bureau's measurement, research, developmental, cooperative, and publication activities, this monthly publication is designed for the industry-oriented individual whose daily work involves intimate contact with science and technology-for engincers, chemists, physicists, research managers, product-development managers, and company executives. Includes listing of all NBS papers as issued. Annual subscription: Domestic, $\$ 6.50$; Foreign, $\$ 8.25$.

\section{NONPERIODICALS}

Applied Mathematics Series. Mathematical tables, manuals, and studies.

Building Science Series. Research results, test methods, and performance criteria of building materials, components, systems, and structures.

Handbooks. Recommended codes of engineering and industrial practice (including safety codes) developed in cooperation with interested industries, professional organizations, and regulatory bodies.

Special Publications. Proceedings of NBS conferences, bibliographies, annual reports, wall charts, pamphlets, etc.

Monographs. Major contributions to the technical literature on various subjects related to the Bureau's scientific and technical activities.

National Standard Reference Data Series. NSRDS provides quantitative data on the physical and chemical properties of materials, compiled from the world's literature and critically evaluated.

Product Standards. Provide requirements for sizes, types, quality, and methods for testing various industrial products. These standards are developed cooperatively with interested Government and industry groups and provide the basis for common understanding of product characteristics for both buyers and sellers. Their use is voluntary.

Technical Notes. This series consists of communications and reports (covering both other-agency and NBS-sponsored work) of limited or transitory interest.

Federal Information Processing Standards Publications. This series is the official publication within the Federal Government for information on standards adopted and promulgated under the Public Law 89-306, and Bureau of the Budget Circular A-86 entitled, Standardization of Data Elements and Codes in Data Systems.

Consumer Information Series. Practical information. based on NBS research and experience, covering arcas of interest to the consumer. Easily understandable language and illustrations provide useful background knowledge for shopping in today's technological marketplace.

\section{BIBLIOGRAPHIC SUBSCRIPTION SERVICES}

The following current-awareness and literature-survey bibliographies are issued periodically by the Bureau :

Cryogenic Data Center Current Awareness Service (Publications and Reports of Interest in Cryogenics). A literature survey issued weekly. Annual subscription: Domestic, $\$ 20.00$; foreign, $\$ 25.00$.

ciquefied Natural Gas. A literature survey issued quarterly. Annual subscription: $\$ 20.00$.

Superconducting Devices and Materials. A literature survey issued quarterly. Annual subscription: $\$ 20.00$. Send subscription orders and remittances for the preceding bibliographic services to the U.S. Department of Conmerce, National Technical Information Service, Springfield, Va. 22151.

Electromagnetic Metrology Current Awareness Service (Abstracts of Selected Articles on Measurement Techniques and Standards of Electromagnetic Quantities from D-C to Millimeter-Wave Frequencies). Issued monthly. Annual subscription: $\$ 100.00$ (Special rates for multi-subscriptions). Send subscription order and remittance to the Electromagnetic Metrology Information Center, Electromagnetics Division, National Bureau of Standards, Boulder, Colo. 80302.

Order NBS publications (except Biblingraphic Subscription Services) from: Superintendent of Documents. Government Printing Office, Washington. D.C. 20.402. 
U.S. OEPARTMENT OF COMMERCE

National Bureau of Standards

Washington, 0.C. 20234

POSTAGE ANO FEES PAIO

OFFICIAL BUSINESS

U.S. OEPARTMENT OF COMMERCE

215

Penalty for Private Use, $\$ 300$ 